\title{
Constitutive modelling of skin ageing
}

Georges Limbert, Damien Pond and Andrew McBride

\begin{abstract}
The objective of this chapter is to review the main biomechanical and structural aspects associated with both intrinsic and extrinsic skin ageing, and to present potential research avenues on how to account for these effects in mathematical and computational models of the skin. This will be illustrated through recent work of the authors which provides a basis to those interested in developing mechanistic constitutive models capturing the mechanobiology of skin across the life course.
\end{abstract}

\section{Introduction}

The skin is not only the largest organ of the human body but is also one of the most complex multi-functional physiological systems in mammalian species [1]. Its main role is to ensure cohesion and protection of the internal body structures against mechanical, thermal, biological and radiological threats. It also has critically important thermoregulation, biochemical synthesis and sensory functions $[1,2]$. Moreover, the skin also plays an important social and psychological role as it is a permanent reminder of our ethnicity, health status, age and past traumas, and therefore, provides direct and indirect cues to the people we interact with. By means of a rich library of mechanically-activated facial expressions, from micro-wrinkles to large macroscopic tissue folds, the skin is a powerful vehicle for conscious and subconscious communication. From these facts it is straightforward to conclude that, beyond physiology, the skin is particularly crucial to human life and could be viewed as a "brain on the outside" [3]. Like any other organ of the body, the skin inexorably undergoes what is termed chronological or intrinsic ageing; a series of biochemical molecular degenerative changes occurring as the result of the mere passage of time and progression into older age. These alterations involve decreased proliferative capacity which leads to cellular senescence and altered biosynthetic activity of skin derived cells. Intrinsic ageing is triggered by two main mechanisms which can operate in concert, namely DNA damage and chromosomes' telomere shortening [4-7]. The fact that the skin of people of identical ages living in similar environments may appear younger- or older-looking is a testimony of the fundamental role of genetics in skin ageing. Genetics conditions the rate of skin ageing by controlling certain factors such as the biochemistry of skin

Georges Limbert

national Centre for Advanced Tribology at Southampton (nCATS) - Bioengineering Science Research Group, Department of Mechanical Engineering, Faculty of Engineering and Physical Sciences, University of Southampton, Southampton SO17 1BJ, United Kingdom Laboratory of Biomechanics and Mechanobiology, Division of Biomedical Engineering, Department of Human Biology, Faculty of Health Sciences, University of Cape Town, Observatory 7935, South Africa, e-mail: G.Limbert@ soton.ac.uk

Damien Pond

Centre for Research in Computational and Applied Mechanics, University of Cape Town, 7701 Rondebosch, South Africa, e-mail: pond@ live.co.za

Andrew McBride

Glasgow Computational Engineering Centre, Division of Infrastructure and Environment, School of Engineering, University of Glasgow, Glasgow G12 8QQ, United Kingdom, e-mail: andrew.mcbride@glasgow.ac.uk 
cells, immunochemistry and hormonal mechanisms. Intrinsic ageing typically occurs in combination with extrinsic ageing which is the result of external environmental and lifestyle factors, particularly exposure to ultraviolet radiations (UVR) from sun light [8-10], and increasingly, sunbeds [11, 12], as well as smoking [13, 14] and air pollution [14, 15]. Extrinsic ageing due to UVR exposure is called photoageing [9, 10], a term which was first coined by Kligman [10] using the American English spelling "aging". These aspects are discussed in more details in Sec. 3.

Ageing affects both the structure and the function of the skin (see Fig. 1). These alterations have important consequences for skin physiology [4, 16], rheology [17], surface physics and tribology [18-20]. Aged skin is more prone to developing disorders such as xerosis and pruritus [21], skin cancers [22] as well as debilitating, costly and lifethreatening skin tears and pressure ulcers [23]. Even in their minor form, these afflictions can severely impact on quality of life for the elderly population. These facts must be considered alongside concerning demographics on the ageing of the population. Between 1984 and 2009 the UK population aged 65 and over increased by 1.7 million people [24]. Between 2001 and 2011, the UK population aged 85 and over increased by almost 25\% (from 1.01 million to 1.25 million) [25]. As of 2016, these two age groups represented respectively 18 and $2.4 \%$ of the UK population [26]. By 2039, almost a quarter of the UK population will be 65 and over [25]. Globally, it has been estimated that the proportion of people over 60 will nearly double from $12 \%$ in 2015 to $22 \%$ by 2050 (i.e. 617 million to 1.6 billion) [27].

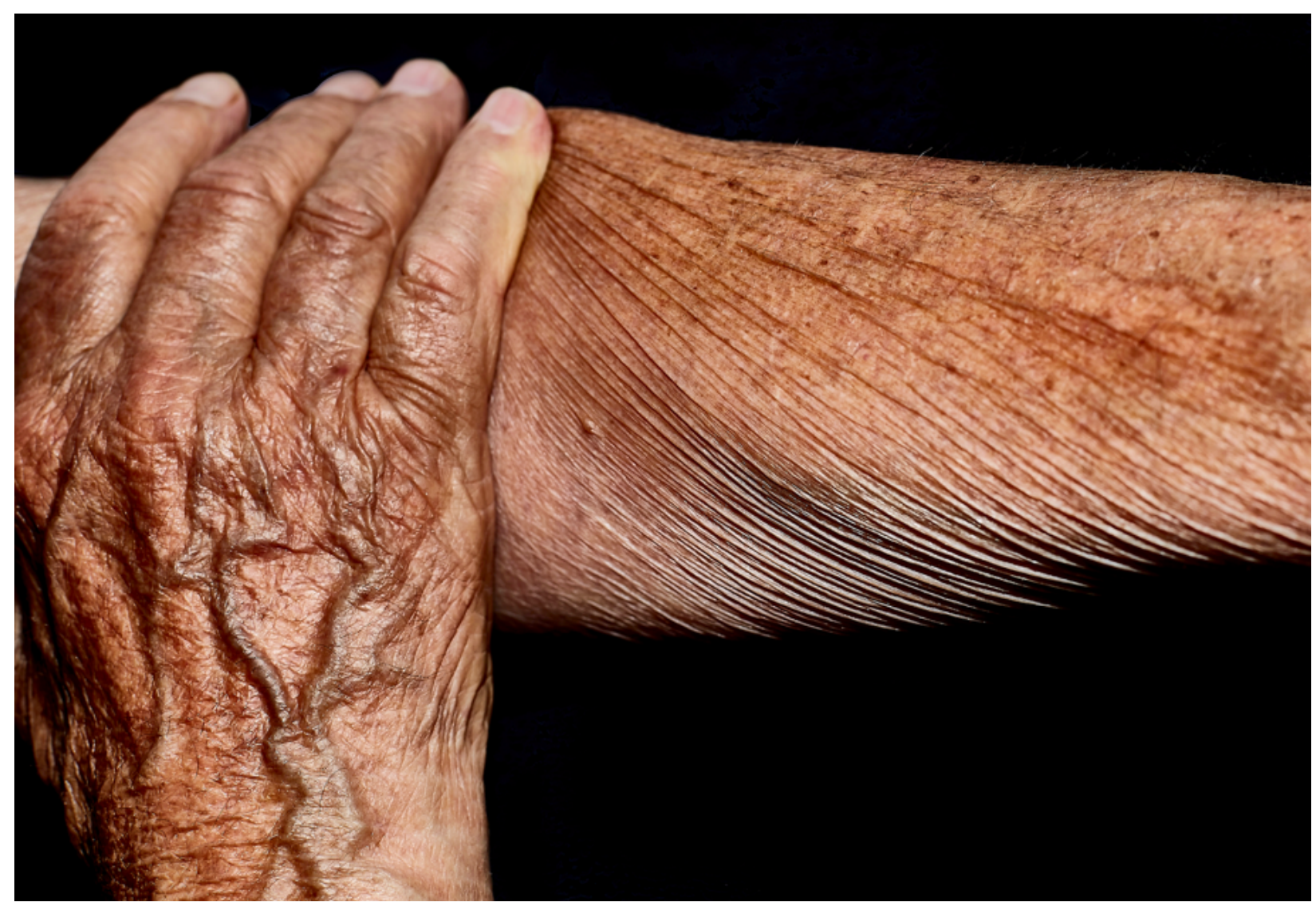

Fig. 1 Left inner forearm and right hand of an 82 year-old White Caucasian male subject with no known skin conditions, highlighting the wrinkled and leathery appearance of both intrinsically and extrinsically aged skin.

The significance of these statistics is embodied by two essential aspects: firstly, the need for medical treatment and care increases with longevity and, secondly, the ageing process itself results in degradation of physiological functions 
and biophysical properties of organs and tissues. In both cases, this puts an alarming economic and social burden on governments and healthcare services [28].

Average hospital spending for an 89 year-old man in the UK is roughly three times the average for a 70 yearold, and nine times the average figure for a 50 year-old, with costs typically escalating more rapidly for men than women [28]. In the case of skin, which accounts for up to $16 \%$ of an adult's total body weight whilst covering an average surface area of about $1.6 \mathrm{~m}^{2}$ [2], ageing can lead to potentially life-threatening complications as mentioned previously in this section. As the skin is the prime line of defence against the external environment, it is clear that any significant alteration of its mechanical properties that could compromise its structural integrity and barrier function, has the potential to cause serious detrimental health effects.

From an economic point of view, both in the UK and globally, the ageing population is also rapidly becoming a significant market segment across many industrial sectors, spanning medical devices through consumer goods and personal care products to sport equipment and consumer electronics. For many of these products (e.g. wearable electronics, razors, incontinence products) the biophysical response of the skin, particularly for contact scenarios, is crucial in terms of comfort, performance and safety. It is therefore essential to engineer products that take into account altered biophysical characteristics of an aged skin (i.e. "inclusive design") so as to optimise their performance in terms of human factors. The goal should be to understand how age-related alterations of skin biophysics can be accounted for in the development of new or improved products that will improve health, quality of life and enable the aged and ageing population to remain active longer. Particular research efforts should be devoted to gain a fundamental and quantitative insight into the mechanisms that drive and govern the ageing process. Unravelling the inherent complexity of the skin ageing process, firstly by identifying its biophysical drivers, underlying modulating factors and effects, and secondly, by gaining a mechanistic insight into their interplay, is a formidable challenge at both experimental and modelling levels. This stems from the factx that:

1. ageing is a multi-factorial problem which features multiple types of processes rooted in biology, chemistry and physics, and more particularly, mechanics;

2. these processes are non-linear and lead to complex non-linear feedback mechanisms;

3. there is a significant intra-individual (primarily due to anatomical location and sun exposure) and inter-individual (as a consequence of age, sex or genetics) variability.

The multi-factorial nature of ageing and the complex non-linear interplay of its biophysical driving factors currently hinder our ability to develop a mechanistic understanding of ageing, and therefore, a rational basis to design prevention and treatment strategies against its degenerating effects.

The provision of mathematical and computational models of skin ageing holds the promise of offering a rational quantitative basis to develop such products whilst also enabling and accelerating innovation, and alleviating the reliance on animal models through a better quantitative understanding of human ageing.

The objective of this chapter is to provide a review of the main biomechanical and structural aspects associated with skin ageing and to present ideas on how to account for ageing in mathematical and computational models of the skin. This will be illustrated through recent work of the authors which could provide a starting point to those interested in developing mechanistic constitutive models capturing the mechanobiology of skin across the life course. Prior to that, some of the more popular theories of skin ageing will be briefly presented.

The chapter is organised as follows. The general structural and material properties of the skin are discussed in Sec. 2. Sec. 3 presents the key manifestations of ageing on the skin from the view point of structural biomechanics. Some of the most popular and accepted theories of skin ageing are also discussed. Finally a simplified mechanistic description of skin ageing unifying essential features of both intrinsic and photoageing is presented in Sec. 4. Background ideas and literature about modelling of ageing from the viewpoint of continuum mechanics are given in Sec. 5. A brief reminder of the essential equations of continuum mechanics necessary to describe the mechanical behaviour of skin is provided in Sec. 6. The structure of the constitutive model used to describe the mechanical response of skin at a fixed moment in time is presented in Sec. 7. Following the recent work of the authors [29], a mechanistic constitutive model of the skin linking microstructural constitutive parameters with intrinsic ageing is described in Sec. 8. The intrinsic ageing model is implemented within the finite element method in Sec. 9 and the constitutive parameters associated with ageing identified. A novel chemo-mechanobiological constitutive model of skin ageing that includes features of both intrinsic and extrinsic ageing is developed in Sec. 10. Finally, Sec. 11 ends with concluding remarks about current and future prospects for the modelling of skin ageing. 


\section{Essential structural and material properties of the skin}

\subsection{Multi-layer nature of the skin}

The human skin is a complex multiscale structure that is often described at the mesoscopic scale as a multi-layer assembly composed of an epidermis, dermis and hypodermis (Fig. 2) [2, 30, 31] (see also Chapter 1 of this book for a more comprehensive description of the molecular and structural properties of the skin).

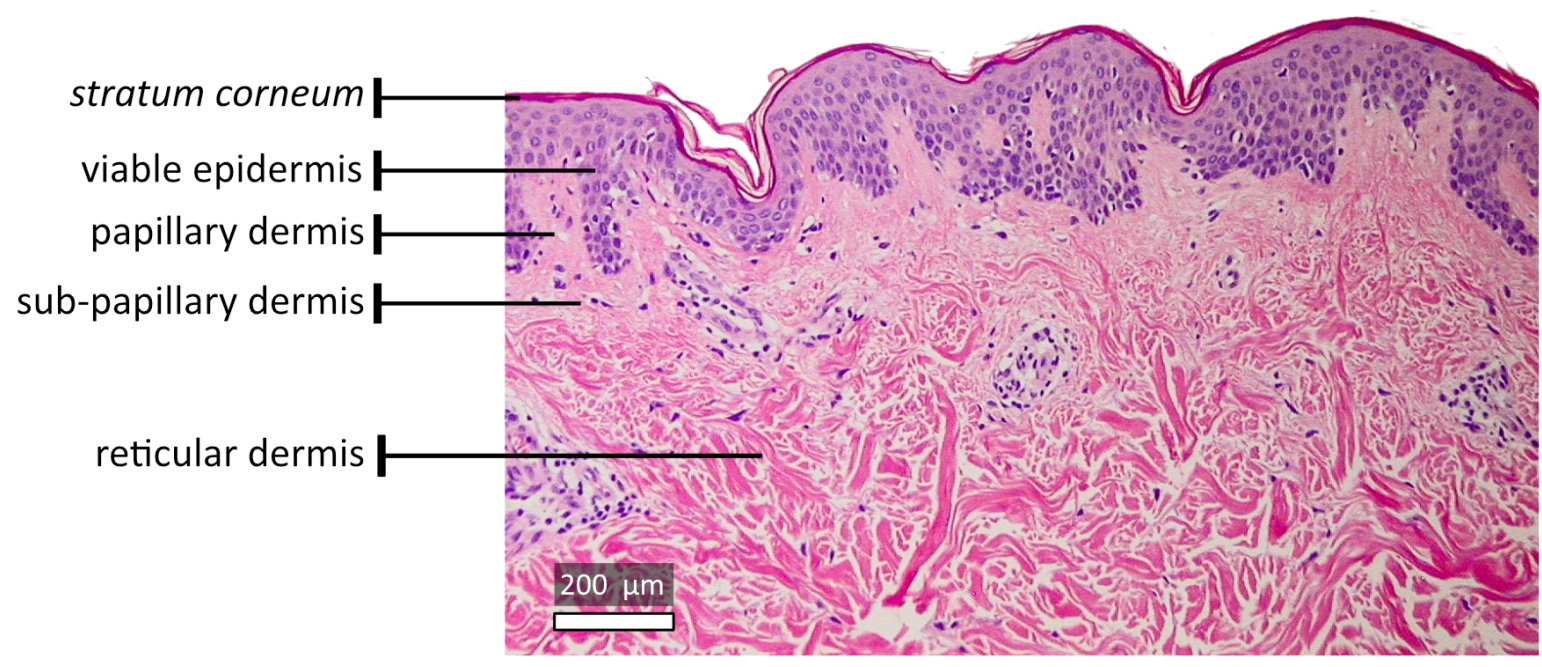

Fig. 2 (a) Histological section of haematoxylin and eosin stained back human skin sample obtained from a 30 years-old healthy White Caucasian female volunteer following biopsy (10 x magnification, image resolution: 1600 x 1200 pixels, imaged using a modified Nikon E950 camera). Image courtesy of Dr. Maria-Fabiola Leyva-Mendivil, University of Southampton, UK. The main skin layers, namely stratum corneum, viable epidermis, papillary dermis, sub-papillary dermis and reticular dermis are indicatedx.

The avascular epidermis is a terminally differentiated stratified squamous keratinocyte-dominated (95\% of its composition) epithelium about $200 \mu \mathrm{m}$ thick which also contains other cell types (e.g. melanocytes, Langerhans's cells and Merkel cells [2]). The epidermis consists of two main substructures, the stratum corneum and the viable, also called living, epidermis (Fig. 2). The stratum corneum consists of a one to three cell-thick layer of dead keratinocytes featuring a $10-30 \mu \mathrm{m}$ total thickness. A $0.5-1 \mu \mathrm{m}$ thick epidermal basement membrane (also known as basal lamina) [32] separates the viable epidermis from the dermis. Keratinocytes undergo mitosis at this location before they progressively migrate toward the skin surface where they die, and eventually form the stratum corneum.

The dermis is 15 to 40 times as thick as the epidermis [2]. It is generally accepted that the bulk of the skin's response to loading is due to the dermal layer $[17,33]$. The extracellular matrix (ECM) of the dermis which is mainly secreted by fibroblasts is constituted of a 3D network of fibrous proteins (mainly type I and III collagen and elastic fibres - namely, elastin and fibronectin) and glycosaminoglycan-rich proteoglycans [34]. The ECM provides strength, extensibility and elasticity to the skin and plays a significant bio-chemo-mechanical role in cell adhesion and regulation of cell signalling.

The expression of the macroscopic mechanical properties of the skin is due to its basic building components, their structural organisation and their mutual interactions. Three distinct zones can be identified within the dermis: the papillary layer juxtaposed to the epidermis, the sub-papillary layer underneath and the reticular layer (Fig. 2) which is connected to the underlying subcutaneous tissue. The papillary layer is defined by the rete ridges protruding into the epidermis and contains thin collagen fibres, sensory nerve endings, cytoplasms and a rich network of blood capillaries. 
The sub-papillary, layer which is the zone below the epidermis and papillary layer, features similar structural and biological components to those of the papillary layer.

The subcutaneous tissue is the layer between the dermis and the fascia which is a band of connective tissue, primarily collagen, that attaches, stabilises, encloses, and separates muscles and other internal organs. The thickness of subcutaneous tissue is highly variable intra- and inter-individually. This layer is mainly composed of adipocytes. Its role is to provide mechanical cushioning, heat generation and insulation as well as a reserve of nutrients.

\subsection{Structural elements of the dermis}

The ground substance is a gel-like amorphous phase mainly constituted of proteoglycans and glycoproteins (e.g. fibronectin) as well as blood and lymph-derived fluids which are involved in the transport of substances crucial to cellular and metabolic activities. Proteoglycans are composed of multiple glycosaminoglycans (i.e. mucopolysaccharides) interlaced with back bone proteins. Dermal fibroblasts produce glycosamine which is rich in hyaluronic acid and therefore play an essential role in moisture retention.

Collagen has been found to make up approximately $66-69 \%$ of the fractional volume of the dermis [17], and approximately 34\% [35] and 70-80\% [36] of the wet and dry weight of the skin, respectively. Experiments where collagen was isolated through enzymatic treatment [37-39], conclude that collagen is responsible for the tensile strength of the skin. Besides a dominant content of types I and III collagen (respectively $80 \%$ and $15 \%$ of total collagen content), the reticular layer is innervated and vascularised, contains elastic fibres (e.g. elastin) and the dermal matrix made of cells in the interstitial space. Cells present in the reticular dermis include fibroblasts, plasma cells, macrophages and mast cells. Collagen fibres in the papillary and sub-papillary dermis are thin (because of their low aggregate content of fibrils) and sparsely distributed while reticular fibres are thick, organised in bundles and densely distributed. Fibrils are typically very long, 100 to $500 \mathrm{~nm}$ in diameter featuring a cross striation pattern with a 60 to $70 \mathrm{~nm}$ spatial periodicity. The diameter of thick collagen bundles can span 2 to $15 \mu \mathrm{m}$. Birefringence techniques have been used to characterise the orientation and supramolecular organisation of collagen bundles in skin [40]. Contributing approximately 2-4\% of the dry weight of skin [41], elastin fibres are highly compliant with the ability to stretch elastically to twice their original length [42]. Their diameter ranges between 1 and $3 \mu \mathrm{m}$. Their mechanical intertwining with the collagen network of the dermis is what gives the skin its resilience and recoil ability. This is evidenced by the correlation between degradation of elastin, abnormal collagen synthesis associated with ageing and the apparent stiffening of the dermis [16]. The diameter of elastic fibres in the dermis is inversely proportional to their proximity to the papillary layer where they tend to align perpendicular to the dermal-epidermal junction surface.

\subsection{Mechanical behaviour of the skin}

At a macroscopic level, the mechanical properties of the skin are anisotropic and inhomogeneous, not only due to the complex hierarchical structure and materially non-linear constituents but also due to the existence of residual tension lines in the skin (i.e. the so-called "Langer lines") as first recognised by the Austrian anatomist Karl Langer in his seminal study [43]. These essential features of skin and their experimental characterisation are discussed in more detail in Chapter 9 of this book.

Dispersion of collagen fibres around the main orientation directions and non-uniform fibre geometry means that under stretch not all fibres are straightened and stretched. This accounts for the anisotropic stiffness response when load is applied either along or across the preferential fibre direction. The magnitude of these directional effects have been the subject of several recent studies. It has been found that the Young's modulus parallel to the Langer lines was greater than that perpendicular by a ratio of approximately 2.21:1 [44]. Similarly, Reihsner et al. [36] found that the degree of anisotropy differs across anatomical site, an observation also made by Langer in his original study. In addition, they found that in situ stresses range from $0.2-1.6 \mathrm{~N} / \mathrm{mg}$ along Langer lines and $0.1-1.3 \mathrm{~N} / \mathrm{mg}$ perpendicularly, with the degree of anisotropy differing between principle stress components from $0.1-0.3 \mathrm{~N} / \mathrm{mg}$. It was shown that when the 
skin is stretched, the elastin fibres are the first to bear load [45], indicating that the contribution is important at low strain levels. Stress-strain curves of elastin-free skin [37] show that elastin supports the entire load up to 50\% strain after which the strength rapidly increases due to the collagen. The elastic modulus of elastin has been found at around $1 \mathrm{MPa}$ which agrees with the Young's modulus of skin at low strain and because elastin is not strong enough to provide much tensile strength at higher strains [46]. Reihsner et al. [36] state that elastin is responsible for the recoiling of the skin and collagen after stress is applied. Following degradation of the elastin through the use of elastase, Oxlund et al. [37] found that the large strain response occurs sooner for a given tensile load. This suggests that in the absence of elastin, collagen fibres take on load at lower strain levels than when elastin is present.

The ground substance has been shown to play a role mainly on the viscoelastic properties of the skin because of high-water content and complex time-dependent interstitial fluid motion. Upon removal of various macromolecules within the ground substance, Oxlund and Andreassen [38] showed that there was no effect on the mechanical response of rat skin, while Oomens et al. [47] suggests that ground substance probably only plays a major role when soft tissue is subject to compression. Under uniaxial tension, skin exhibits a typical strain-hardening response, featuring three main characteristic zones (a), (b) and (c). Each portion of the stress-strain curve can be explained by particular structural deformation mechanisms associated with the skin dermal constituents. (a) Low modulus portion of the strain-stress curve: this occurs over the gradual straightening of crimped collagen fibres. During this stage, the greatest resistance to loading is generated by the elastin and ground substance, with collagen fibres offering very little resistance. The low modulus portion can be further divided into two phases: (1) Phase 1: wavy collagen fibres are still relaxed and elastin fibres take on the majority of the load; (2) Phase 2: collagen fibres start to uncrimp, then elongate and eventually start to bear load; (b) Linear region of the strain-stress curve: collagen fibres straighten and align with the load direction. Straightened collagen fibres strongly resist loading. This results in the rapid stiffening of the skin. The steep linear stress-strain relation is due to stretching and slippage between fibrils and molecules; (c) Final yield region of the strain-stress curve: tensile strength of collagen fibres is reached and fibres begin to sequentially break.

\section{Manifestations of skin ageing and underlying biophysical mechanisms}

It is often thought that what one refers to as ageing of the skin is mostly due to the bio-structural alterations of the skin induced by extrinsic ageing, as a "well-maintained" skin, in terms of appropriate diet and skin care, and protected from UVR, exhibits a "remarkable resilience" [48] to intrinsic ageing. Intrinsic ageing can only be observed in old age subjects, and its extent and magnitude is strongly dependent upon ethnicity, individuals, even within the same ethnic group, and body locations. It is also characterised by a very gradual evolution of the skin appearance over decades, unlike extrinsic ageing which can take place over much shorter periods of time. As would intuitively be expected, both intrinsic and extrinsic ageing operate in concert and influence each other [48]. For example, exogenous factors associated with a particular environment such as pro-oxidants and antioxidants have an impact on cell turnover through neuro-endocrine-immune biological response modifiers. While intrinsic ageing can be influenced by extrinsic ageing it could also be defined as a form of purely biological ageing, a process genetically determined and immutable [49]. Following a similar classifying logic, factors leading to extrinsic ageing can be split into three main types: environmental (e.g. effects of UVR, chemical pollution including that induced by the use of tobacco products, temperature), mechanical (e.g. repetitive muscle actions leading to tissue plasticity such as squinting and frowning) and lifestyle (e.g. diet and sleep patterns).

Intrinsic ageing is characterised by unblemished, smooth, stiffer, drier and less elastic skin [48] with fine wrinkles, with occasional exaggerated expression lines [34, 50], epidermal and dermal atrophy as well a reduction in the population of mast cells [51]. Extrinsic skin ageing manifests as deep wrinkles and leathery appearance due to photo-damage, pigmented lesions, actinic keratosis and patchy hyperpigmentations [52, 53]. The externally visible structural effects of extrinsic ageing on the skin can be viewed as exaggerated intrinsinc ageing effects. It is worth to point out that UV exposure leads to additional photobiochemical effects which are not present in intrinsic ageing. Extrinsic ageing is most apparent on sun-exposed body locations such as the face, neck, chest and the dorsal surface of the arms [8]. It is estimated that $80 \%$ of the effects of facial skin ageing are due to chronic UV exposure. 
A review of theories of skin ageing is out of the scope of the present chapter and the reader is referred to recent excellent review papers such as those by Gragnani et al. [53], Tobin [48] and Krutmann et al. [54] for detailed descriptions of established and suspected ageing mechanisms. There is very strong evidence that the main factors involved in skin ageing are oxydative stress (accumulation of oxidative damage to cells during their life due to excessive production of reactive oxygen species (ROS)), cellular senescence and telomeres' shortening due to apoptosis, diet, genetics, UV irradiation, smoking, pollution, intracellular signalling and skin lesions, age-related diseases, disorders and conditions of the skin, hormonal changes and the production of advanced glycation end products (AGEs) [53].

\subsection{Ageing of the skin considered as a homogeneous structure}

It is generally accepted that skin thickness decreases with age [39, 55]. Pawlaczyk et al. [56] found that there is an overall loss of 0.7-0.8 mm of thickness in older skin. It was found that skin thickness reaches a maximum around the fourth decade for men and third decade for women after which there is a gradual decrease [35], a result similar to that established by Diridollou et al. [57] who found that after an initial increase during maturation ( $0-20$ years), thickness remains constant to about the age of 60 followed by a decrease according to the following equation:

$$
t=-6 \times 10^{-3} \times \text { age }+1.3,
$$

where $t$ is in $\mathrm{mm}$ and the age is in years. Moreover, the rate of decrease is more significant in female subjects.

It is important to highlight that these observations about skin thickness apply to the skin as a whole composite structure. Individual skin layers may follow different trends depending on whether intrinsic and extrinsic ageing effects are considered separately or assumed to be combined. After 20 years of age, across all layers, the skin thickness starts to diminish at a rate that increases with age [58]. Between 30 and 80 years, the unexposed skin can lose up to 50\% of its thickness and this effect is accentuated in zones exposed to sunlight such as the face or neck. Overall, epidermal thickness drops by about $6.4 \%$ per decade, and at a faster rate in women than men. It is generally believed that the reported reduction in dermal thickness is mainly caused by the loss of dermal collagen and elastin in elderly adults [59]. It was shown that in post-menopausal women a $1.13 \%$ per year skin thickness reduction is correlated with a $2 \%$ decrease per year in collagen content [60]. Besides decreased elastin and collagen content as well as their structural rearrangement, intrinsic ageing also has other consequences such as increase in trans-epidermal water loss, reduction in skin moisture content, diminished sebum production, arteriosclerotic changes in the small and large vessels, thinning of vessel walls [61], reduction in mast cells [51], melanocytes, Langerhans's cells, Meissner cells, Merkel cells and Pacinian corpuscules [61, 62] and increase in skin surface $\mathrm{pH}$ [63] after 70 years of age [64].

It is also accepted that skin ageing is characterised by an increase in macroscopic or apparent stiffness $[56,65,66]$, although there is little agreement on the magnitude of the ground state Young's modulus and age of onset of stiffening. As mentioned by $\mathrm{Xu}$ and $\mathrm{Lu}$ [66], there is a sudden increase in the Young's modulus of the skin at age 30 of around $50 \%$, whereas others quote an increase from the age of 45. It was observed by Escoffier et al. [55] that there is an increase of around $20 \%$ after the age of 70 , which is backed by the findings of Lévêque et al. [35]. Furthermore, Alexander and Cook [65] found that the stiffness of skin starts to increase from the age of 25 but noted that the variation in results increases with age. This suggests that the process of skin ageing is a highly patient specific and may explain the large variation in results reported in the literature.

It was found by Escoffier et al. [55] and Lévêque et al. [35] that intrinsic skin extensibility (i.e. a standardised mean extensibility to account for varying skin thickness) decreases with age, while Alexander and Cook [65] found that intrinsic extensibility decreases by around $35 \%$ after the age of 65 which agrees with the findings of $\mathrm{Xu}$ and $\mathrm{Lu}$ [66] in that maximum skin elongation is found between ages 35 and 55. Similarly, skin elasticity (i.e. recoil ability) decreases with age [55, 66], which was similarly found by Henry et al. [67], but this may include the effects of UVR. 


\subsection{Ageing of the epidermis}

Intrinsic ageing induces thinning of the epidermis whilst also slowing its turnover. However, photoageing is manifested as a thickening of the epidermis because of its disruptive action on keratinocyte differentiation which leads to abnormal keratin intermediate filament expression. If one then superimposes the drying effect of photoageing on the stratum corneum, a reduced rate of desquamation is observed. As a consequence, the epidermis becomes thicker, stiffer and more fragile [68]. Overall, epidermal thickness drops by about $6.4 \%$ per decade at a faster rate in women than men [58]. Based on the observation that in areas of skin under high levels of external loading, such as on the hands and feet, the epidermis thickens, Silver et al. [69] suggested that epidermal thickness is controlled by the balance of external and internal forces acting on/within this layer. Intrinsic ageing slows down the turnover of keratinocytes and is also accompanied by a reduction in stratum corneum water content [70] because of alteration in lipid content of the epidermal barrier [71]. This factor leads to an increase in the stiffness of the stratum corneum which is correlated with humidity level [72-74]. For the skin to be considered clinically dry the normal water content of the stratum corneum (about 20\%) must drop below 10\% [70].

\subsection{Ageing of the dermal-epidermal junction (DEJ)}

In young skin, there is an essential network of thin oxytalan fibres anchoring the papillary dermis to the viable epidermis. During intrinsic ageing, these oxytalan fibres are progressively shortened and resorbed leading to the disappearance of dermal papillae [75] and flattening of the dermal-epidermal junction (DEJ) [76-80]. This reduction in the amplitude of the papillae is also accompanied by a decrease in their density (unit per surface area) [77, 78]. The flattening of the DEJ leads to a reduction of up to $20 \%$ in epidermis thickness [81]. It was suggested that the flattening of the DEJ could be a facilitating factor in the formation of skin wrinkles by compromising the structural integrity of the basement membrane which links dermis to epidermis [82], increasing the likelihood of relative motion between these two layers. Besides these important structural effects, the flattening of the DEJ has important metabolic consequences as it reduces the surface area for nutritional exchange and metabolic byproducts evacuation between the dermis and epidermis. As a result, epidermal cell turnover is slowed down and free radicals accumulate. Skin exposed to UVR experiences an accelerated flattening of the DEJ when compared to sun protected skin [83]. It has also been shown through image analysis that intrinsically aged skin features the same density of veins and arteries as young skin but their diameters are reduced. In photoaged skin, the dermal vasculature is progressively lost and the diameter of veins and arteries is also reduced [84].

\subsection{Ageing of dermal matrix}

Important differences between the dermis of young, intrinsically aged and photoaged skin pertain to the level of structural organisation of fibrillar collagen which conditions its mechanobiological interactions with fibroblasts through their integrin attachments [34]. In young skin, the fibrillar collagen meshwork is made of small and thin bundles of tightly packed fibres in the papillary dermis while these bundles are thicker and more spaced in the reticular dermis $[76,85]$. Fibroblasts are in a state of mechanical tension through the alignment along collagen fibres, the effect of which promotes a healthy homeostatic state for normal collagen fibre synthesis. The ratio of type III to I collagen increases with age $[86,87]$. In intrinsically aged skin, there is a significantly reduced collagen turnover, thinning of fibre bundles and disappearance of the meshwork [88]. These structural changes affect the mechanobiological interactions of fibroblasts with collagen fibres leading to collapsed fibroblasts [88-90].

Lavker et al. [91] reported an increased density of the collagen network with age and explained it by the decrease in ground substance which effectively provides more space to fill in to collagen fibres. This has the effect of reducing dermis thickness. With age, the collagen and elastin fibre networks in the dermis become compacted leading to reduction in dermis thickness. Elastin fibres lose their elasticity while collagen fibres tend to unravel. As a consequence, 
the skin loses its extensibility, becomes less resilient and more lax [92]. The reported reduction in dermal thickness is mainly attributed to the loss of dermal collagen and elastin in elderly adults, as a result of progressive slowing down of dermal matrix turnover [59]. This reduced turnover stems from the imbalance between synthesis activity and degradation which increasingly dominate cell activity during the intrinsic ageing process.

The key regulators of collagen production are transforming growth factors- $\beta$ (TGF- $\beta$ ) and activator protein-1 (AP1) [93]. TGF- $\beta$ is a multi-functional cytokine that helps regulate many biological functions such as cellular growth, differentiation and extracellular matrix synthesis, such as that associated with collagen and elastin constituents [94]. AP-1 is a transcription factor that inhibits collagen production and up-regulates matrix metalloproteinase (MMP) enzymes which act to break down extracellular matrix constituents. All fibroblasts have the capacity to synthesise collagen. Thus one of the main contributing factors to collagen synthesis is the proliferative capacity of resident fibroblasts. Synthesis of collagen is promoted through mechanical tension applied to dermal fibroblasts. The rate of collagen synthesis is conditioned by the level of mechanical tension [11].

Platelet derived growth factor (PDGF) is a substance that promotes fibroblast growth and proliferation, known as mitogen. PDGF binds to cell surface receptors, which activates mitogen-activated protein (MAP) kinase signalling. The result of this action is the signalling of downstream effectors, such as the activation of extra-cellular signal-related kinase (ERK), that promote cell growth and proliferation [93]. TGF- $\beta$ is produced within the extracellular matrix and, when in active form, is able to associate with TGF- $\beta$ signalling receptors on cell surface of dermal fibroblasts, referred as type I and type II receptors, or T $\beta$ RI and T $\beta$ RII respectively. This results in the activation of downstream pathways, notably the Smad protein pathways. Smads 2,3 and 4 transduce the signals while Smad 7 acts as an inhibitor. Together with interaction with MAP kinase signalling, collagen synthesis is promoted [94].

The intrinsic ageing process is accompanied by a reduction of the fibroblast population [51] which induces a decrease in collagen production and is associated with an increased build-up of MMPs which can cleave elastic fibre molecules [93, 95]. MAP kinase activation is an important mediator of MMP production. Along with activation of the ERK pathways, signalling the jun-N-terminal kinase (JNK) are necessary for the regulation of MMPs. Activation of the JNK pathways results in the production of c-jun, while ERK signalling results in the production of c-fos, both of which are necessary for the production of AP-1. AP-1 is key to the production of MMP. There exists a class of proteins, known as tissue inhibitors of metalloproteinases (TIMPs), that control inhibition nd regulation of MMPs. TIMP-1 is prominent in the skin. TIMPs are also regulated by downstream signalling through the MAP kinase. A simplified schematic representation of the key cellular mechanisms associated with dermal fibroblasts in intrinsic ageing is provided in Fig. 3.

Disruption of the homeostatic state between activation and inhibition of MMPs is a key ingredient in the pathophysiology of both intrinsic and extrinsic ageing. The ratio of type III to I collagen increases with age [86]. Macroscopically, alterations of the collagen network manifest themselves as reduced dermal volume and strength. Moreover, aged collagen fibres undergo non-enzymatic Maillard reactions that cross-link molecules by glycation [96, 97], and lead to non-degradable abnormal fibres [85] which belong to the class of advanced glycation end products (AGEs).

Chronic exposure to UV radiations induces a significant and incomplete ECM degradation. In photoaged skin, there is a massive increase in collagen fibre degradation. Acute UV exposure activates a key transcription factor in cells, namely AP-1, which triggers an increase in MMP 1, 2, 3 and 9 synthesis and cell activity linked to a decreased in type I procollagen synthesis [98]. It has also been suggested that via the inflammatory response associated with chronic UV exposure, immune cells could play a role in collagen network degradation [8,99]. During the intrinsic ageing process collagen becomes less soluble, sparser and thinner while photoageing induces thickening, fragmentation and an increased solubility of collagen [87].

Elastin fibres are highly compliant with the ability to stretch elastically to twice their original length [42]. Their mechanical intertwining with the collagen network of the dermis is what gives the skin its resilience and recoil ability, by maintaining collagen fibres in a crimped state. This is evidenced by the correlation between degradation of elastin, abnormal collagen synthesis associated with ageing and the apparent stiffening of the dermis [16, 55]. It has been widely observed that, with age, there is a decrease in the initial portion of the elongation-stress curve of skin [36, 65, 100] which means that the onset of stiffening associated with recruitment of collagen fibres occurs at lower stretch. This is attributed to degradation of the elastin network and deposition of amorphous elastin $[17,36,65,100]$.

It has been reported that the slope of the linear portion of the elongation-stress curve tends to increase with age [65]. This suggests an apparent stiffening of the collagen fibre network with age but not necessarily a stiffening of 

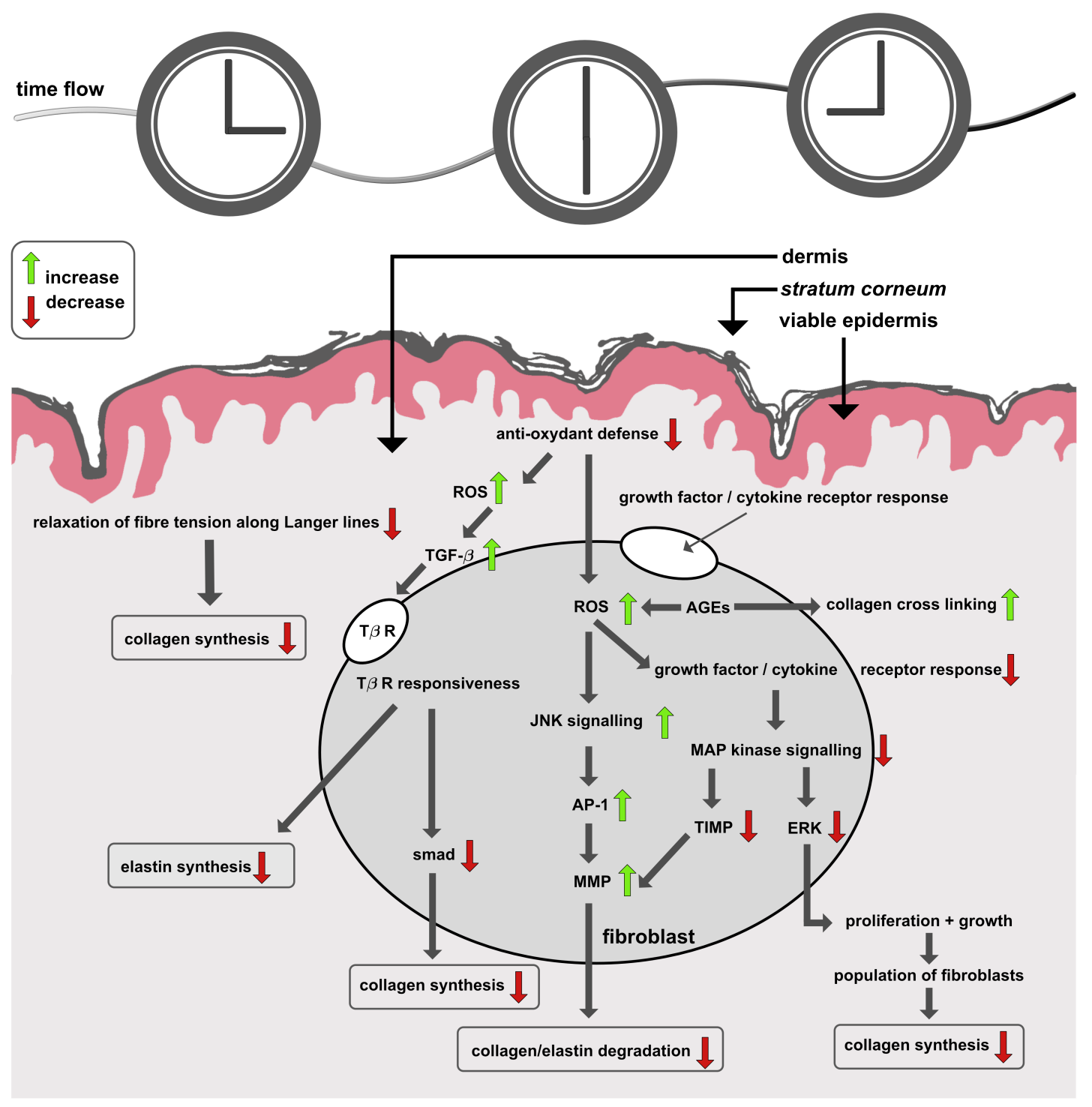

Fig. 3 Simplified schematic representation of the key cellular mechanisms associated with dermal fibroblasts in intrinsic ageing. A green/red arrow indicates an increase/a decrease. [ROS: Reactive Oxygen Species; TGF- $\beta$ : Transforming Growth Factor $\beta$; T $\beta$ R: TGF- $\beta$ receptor; AGE: Advanced Glycation End product; JNK: Jun-N-terminal-Kinase; MAP: Mitogen Activated Protein; MMP: Matrix Metalloproteinase ; TIMP: Tissue Inhibitor of MetalloProteinase; AP-1: Activator Protein-1; ERK: Extracellular signal-Related Kinase].

the collagen fibres themselves. This aspect is supported by the experimental evidence obtained by Daly and Odland [100] and Reihsner et al. [36] who note that the final slope of the strain-stress curve of skin in tension remains constant with age, and that the stiffness of the collagen remains constant, possibly due to the reduction in the collagen content in the dermal layer. There is therefore a suggestion that the alteration of the skin's apparent stiffness is not due to a stiffening of the collagen fibres, but rather to an alteration in the structure of the collagen network [29]. Escoffier et al. [55] and Reihsner et al. [36] report an increased collagen crosslinking with age, which would reduce any slippage between neighbouring fibres and stiffen up the collagen network. This would support the observations of Batisse et al. [72] who reported a stiffening with age of the whole dermis while noting a reduced density in the collagen fibres of 
the papillary dermis of aged skin as this might suggest that other stiffening mechanisms may be at play (e.g. increased collagen cross-linking with age [101]).

Anisotropy tends to increase with age suggesting that there is an increase in alignment along Langer lines with age $[102,103]$, or at least, a correlation between age, microstructurally-induced anisotropy and Langer lines, although Tonge et al. [41] found a decrease in overall anisotropy with age. The magnitude and directions of the Langer lines [43] are known to vary with age. There is a reduction in magnitude with age which is linked to the thinning of the hypodermis leading to a loss of firmness of the skin [101]. Also, with age, the skin tends to lose its in-plane isotropy [102] because of the strong mechanical effects introduced by dermal collagen realignment arising in combination with collagen cross-linking and density alteration.

Chronic UV irradiation spanning many years alter the normal structural and mechanical characteristics of the skin and ultimately causes premature skin ageing and cancer $[8,104]$. Acute exposure to UV radiation sources trigger photochemical reactions in the skin which can manifest as sunburn, inflammation, immunity suppression, modified pigmentation and dermal connective tissue damage [104]. The photons carrying radiative energy quanta (of various wave lengths) are absorbed by molecules named chromophores in the skin where they create states of energetic excitations which engender the aforementioned photochemical reactions. These reactions induce the formation of stable photoproduct molecules [104] which, in turn, stimulate cellular signal transduction pathways which control cell proliferation and apoptosis, and also the secretion of cytokines. For most responses to UV and visible light exposure, the magnitude of the response is conditioned by the exposure dose at a given wave length. Wavelengths of radiations emitted by the sun shorter than $290 \mathrm{~nm}$ are mostly absorbed by the ozone layer in the stratosphere and do not reach sea level. Wavelengths in the range of 200 to $290 \mathrm{~nm}$ are strongly absorbed by DNA and therefore can be lethal to viable cells of the epidermis [104]. The UVB wave band (280 to $315 \mathrm{~nm})$ is often referred as mid-UV or sunburn spectrum and constitutes about $0.5 \%$ of the total radiation reaching the Earth's surface. Most sunscreen agents reflect or absorb radiations operating in this waveband which is the testing benchmark upon which the sun protection factor (SPF) is based. Within the UVB spectrum, certain wave lengths have drastically different effects: radiations at $297 \mathrm{~nm}$ are nearly 100 times more erythemogenic than $313 \mathrm{~nm}$ radiations [105] and more effectively cause DNA damage and photocarcinogenesis [106]. Of the total amount of UV radiations reaching the Earth's surface, about 95\% is made of UVA waves ( 315 to $400 \mathrm{~nm}$ ). The UVAII band $(320-340 \mathrm{~nm})$ is more damaging to unsensitised skin than the shorter wave length band UVAI (340-400 nm).

Extrinsic ageing through UV exposure leads to a partial degradation of existing elastic fibres by fibroblast and neutrophil elastase from the inflammatory infiltrate [107]. This is accompanied by higher turnover of tropoelastin and abnormal synthesis of new fibres [108].

The combined effects of the lysis of existing elastic fibres and synthesis of abnormal and non-functional elastic constituents provokes the accumulation of an amorphous and dense elastotic material in the upper and mid-dermis. The process leading to this altered quality of dermal tissue is called actinic damage [104, 109] which, superimposed on the loss of normal elastic fibres, has a drastic effect on the recoil capacity and resiliency of the dermal layer [110]. In photoageing, glycosaminoglycans (GAGs) which are key to skin hydration [111] are abnormally located on the elastotic material in the superficial dermis instead of being more uniformly distributed in the whole dermis. Moreover, hyaluronic acid (HA) and proteoglycans (versican and decorin) undergo structural alterations with age which lead to impairment of their water retention abilities [104]. A simplified schematic representation of the key cellular mechanisms associated with dermal fibroblasts in photoageing is provided in Fig. 4.

\section{A mechanistic description of both intrinsic ageing and photoageing}

It is clear that both intrinsic and extrinsic ageing are extremely complex coupled biophysical processes which involve a wide range of biochemical species and interacting cellular processes. Their effects are also modulated within the skin microstructural constituents which naturally encompass a great intra- and inter-individual variability. In order to develop a tractable mechanistic model of ageing it is critical to first extract some of their essential (i.e. dominant) features. Generally, using a simplified view, two main theories of skin ageing can be put forward: cellular senescence theory of ageing and free radical theory of ageing 


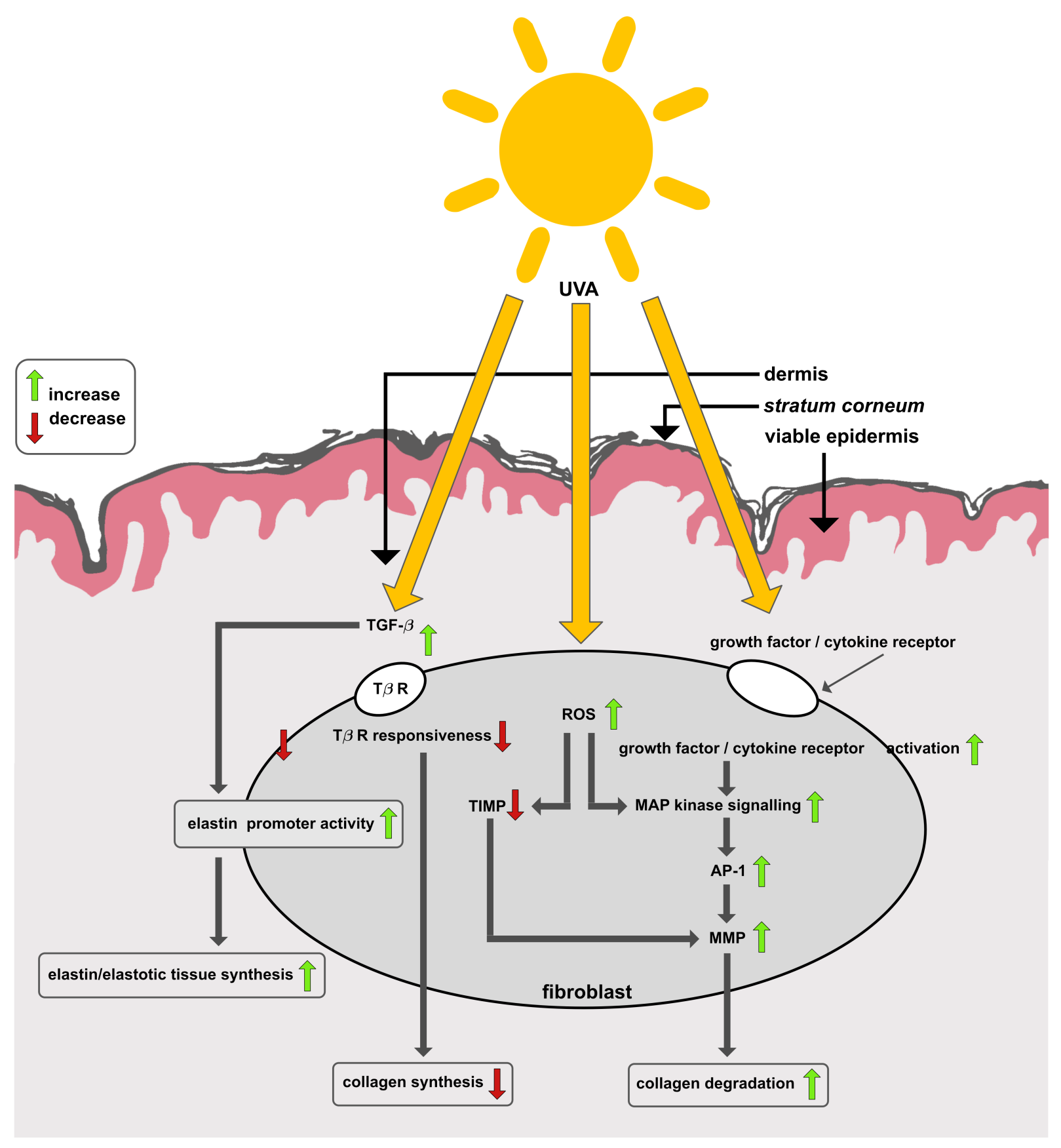

Fig. 4 Simplified schematic representation of the cellular mechanisms associated with dermal fibroblasts in photoageing. A green/red arrow indicates an increase/a decrease. [ROS: Reactive Oxygen Species; TGF- $\beta$ : Transforming Growth Factor $\beta$; T $\beta$ R: TGF- $\beta$ receptor; AGE: Advanced Glycation End product; JNK: Jun-N-terminal-Kinase; MAP: Mitogen Activated Protein; MMP: Matrix Metalloproteinase; TIMP: Tissue Inhibitor of MetalloProteinase; AP-1: Activator Protein-1; ERK: Extracellular signal-Related Kinase]. 


\subsection{Cellular senescence theory of ageing}

According to this theory, the ageing process is a result of a combination of decreased cell proliferation ability, decreased matrix synthesis and increased expression of degrading enzymes of the collagenous matrix [112]. It has been shown that keratinocytes, fibroblasts and melanocytes all display an age-associated decreased in proliferative ability and irreversible cell arrest, which is a process termed as cellular senescence.

Essentially, cellular senescence is a change in the state of a cell, and not a deterioration of cell capabilities with time. Cells undergo a change that reduces, or completely arrests, cell growth and proliferation. Cell division plays a role in the gradual loss of replicative ability. On each cell division, there is a shortening of cellular telomeres. Telomeres are the end bits of DNA strands that have been shown to play a crucial part in ageing. This gradual change alters the physical expression of a cell's genotype, known as its phenotype [113]. As observed in senescent fibroblasts, there is a selective repression of genes involved in growth regulation. As a consequence, this change results in the end of the replicative lifespan as the cell is no longer able to enter the first stages of mitosis. In pre-senescent fibroblasts, the level of MMP-1 and MMP-3 have been observed to expressed at very low levels. Additionally, TIMP levels have been shown to be high, further reducing MMP expression. The is reversed in senescent skin, where there is increased MMP and reduced TIMP expression. Coupled to this is the decreased rate of collagen synthesis in older skin, which further compounds the observed disorganisation and reduced presence of dermal collagen.

\subsection{Free radical theory of ageing}

An alternate, and more popular view of ageing is that due to oxidative stress. Ageing results as an accumulation of oxidative damage due to a build-up of ROS. This ROS production is a result of aerobic metabolism. There has been signifcant research that supports the role of ROS and cumulative oxidative damage as a major contributor to the ageing process $[11,112,114]$. The main source of ROS in ageing is through mitochrondrial oxidative energy generation. Over the course of ageing, damage accumulation due to ROS results in reduced antioxidant capacity through mitochrondrial deterioration which furthers ROS generation. This view of ageing is backed up by the observed higher levels of ROS in aged skin. Over the last few years there have been several conflicting results in studies on the effects of ROS [115]. For example, it was found that increased ROS may prolong the lifespan of yeast cells and that of Caenorhabditis elegans (a nematode), genetic manipulations in mice to increase mitochrondrial ROS do not accelerate ageing, and mice with induced increased antioxidant defence do not experience an increased lifespan. There has also been research in the field of intracellular signalling that provides evidence that the role of ROS may in fact be as a survival response to physiological signals.

\subsection{A mechanistic model of both intrinsic and photoageing of the skin}

In order to consolidate the theories of ageing discussed in this section with the damaging properties of ROS established in previous studies, the following hypothesis is proposed and adopted for the development of the chemomechanobiological model of skin ageing as described in Sec. 10.

The primary effect of ROS is to trigger homoeostatic responses, such as cellular proliferation, in response to physiological stress. As chronological age advances, cellular damage increases while ROS levels increase as well in an attempt to maintain survival. Beyond a certain threshold, ROS eventually start to aggravate age-associated damage. Whether the traditional theory of oxidative damage is adhered to or whether this new hypothesis is adopted, the cellular processes are the same. As a person ages, cells undergo a morphological change similar to that described in cellular senescence. Through the accumulation of ROS, the structural and functional capabilities degenerate, which only accelerates with advancing age. In skin, fibroblasts undergo this morphological change from mitotic cells to ones that are no longer able to undergo mitosis. This change is likely, as with senescence, to alter the phenotypical behaviour of 
the cell, thus negatively affecting type I procollagen production. Additionally, as mentioned, ROS has a direct impact on dermal collagen through the upregulation of AP-1 and MMPs

There is further evidence to support reduced antioxidant activity within the skin. Although results have been conflicting, in general they show that there is a reduction of antioxidant enzymes with age. It has also been demonstrated that aged fibroblasts are far more susceptible to the accumulation of oxidised proteins following oxidative stress, whereas young fibroblasts were able to remove these proteins more effectively [116].

Although the theory of cellular senescence has value, the free radical theory of ageing is thought to be far more relevant in terms of ageing in skin. By adopting this theory, a convenient and logical link is established between intrinsic ageing and photoageing (Fig. 5). Increased ROS production is a common biophysical process to both intrinsic ageing and photoageing. If this process - or its direct/indirect effects — can be modelled, then its influence on synthesis and degradation of two fundamental building blocks of the skin dermis affected by ageing can be approximated, namely collagen and elastin, and one is provided with a basis to formulate mechanistic constitutive equations of skin ageing that would link ageing, skin microstructure and macroscopic mechanical properties of the skin. This will be described in more details in subsequent sections.

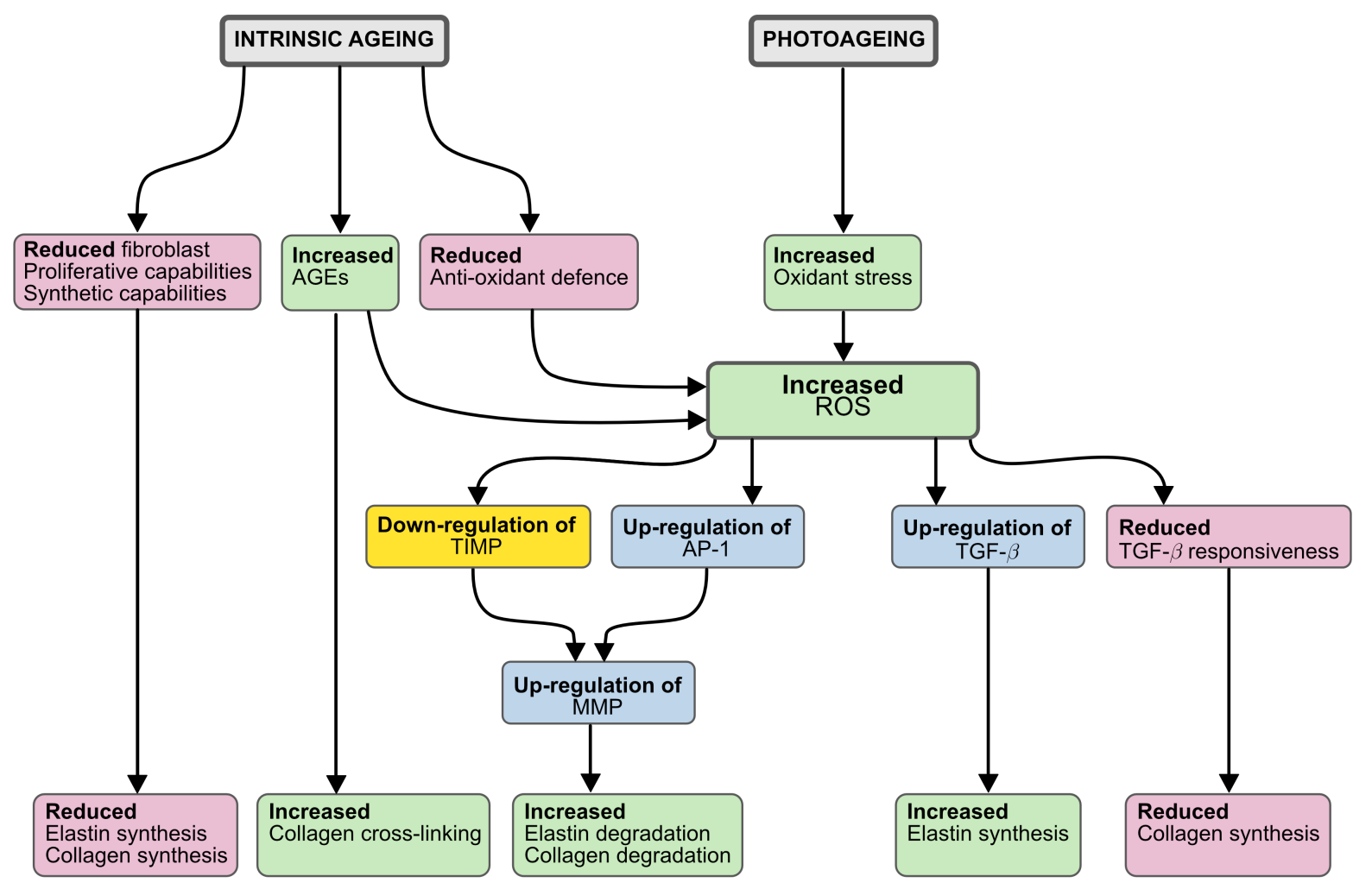

Fig. 5 Schematic and simplified representation of the underlying mechanisms and effects associated with the free radical theory of skin ageing in terms of collagen and elastin. [ROS: Reactive Oxygen Species; TGF- $\beta$ : Transforming Growth Factor $\beta$; T $\beta$ R: TGF- $\beta$ receptor; AGE: Advanced Glycation End product; MMP: Matrix Metalloproteinase; TIMP: Tissue Inhibitor of MetalloProteinase; AP-1: Activator Protein-1] 


\section{Modelling of skin ageing}

As a result of biochemical changes associated with ageing, the mechanical behaviour of the skin is significantly altered through time-dependent variations in the structural and mechanical properties of its elemental constituents (e.g. proteoglycans, collagen, elastin and keratin). The effects of skin ageing become apparent under the form of skin wrinkles - particularly on the face and hands - which are often unconsciously or consciously used as criteria to evaluate somebody's age. These morphological changes of the skin surface are manifestations of complex coupled biophysical phenomena where mechanics is believed to play a critical role like in many other remodelling and morphomechanical processes $[117,118]$. Unravelling the inherent complexity of the skin ageing process, firstly by identifying its biophysical drivers, underlying factors and effects, and secondly, by gaining a mechanistic insight into their interplay, is a formidable challenge at both experimental and modelling levels. However, building upon the solid body of work on the constitutive modelling of the skin [119-121], there are opportunities to extend existing models and develop mechanistic constitutive formulations that could, first describe, and ultimately, predict ageing effects. These hypothesis-driven research tools have the potential to enable us to unveil the biophysical complexity of skin physiology as well as mechanobiological aspects associated with diseases and the ageing process. Various phenomenological and structurally-based mechanical models have been proposed and adopted to model the skin at finite deformations. For a good coverage of the relevant literature see the monograph by $\mathrm{Xu}$ and $\mathrm{Lu} \mathrm{[66],} \mathrm{review} \mathrm{papers} \mathrm{by} \mathrm{Jor} \mathrm{et} \mathrm{al.} \mathrm{[120],}$ Li [121] and Limbert [119] or book chapters by Flynn [122] and Limbert [30]. Structurally-based models attempt to reflect the contributions and mutual interactions from the primary constituents of the dermal layer, i.e. collagen, elastin and ground substance. Developing this type of models is desirable as it offers the ability to link the microscopic constituent characteristics (i.e. materials and structures) to the macroscopic response of the tissue [see 123-125].

Mazza et al. [126, 127] developed a non-linear constitutive model to simulate ageing of the human face. The elasto-visco-plastic constitutive equations are based on the constitutive formulation of Rubin and Bodner [128] to model the dissipative response of soft tissues. The dissipative material model was implemented in a commercial finite element code to simulate gravimetric descent of facial tissue. Mazza et al. [126, 127] extended the model of Rubin and Bodner [128] by including an ageing parameter equipped with its own time evolution equation. This ageingdriven parameter was a modulator of tissue stiffness. A four-layer model of facial skin combined with a face-like geometrical base was developed and highlighted the usefulness of such a model to study the effects of skin ageing on facial appearance. Maceri et al. [129] proposed an age-dependent multiscale mechanical model for arterial walls that effectively coupled elastic nanoscale mechanisms linked to molecular and cross-link stretching to micro- and macroscopic structural effects. The model successfully captured the age-dependent evolution of arterial wall mechanics through alterations of its constitutive parameters including geometric characteristics of collagen fibres, cross-link stiffness of collagen fibrils and volume fraction of constituents.

However, to date, with the exception of the recent work of the authors [29], no mechanistic constitutive models for the skin has been developed that is capable of simultaneously capturing intrinsic ageing through evolution of its material and structural constitutive parameters whilst being embedded in the rigorous framework of non-linear continuum mechanics. The goal of the research presented in the paper by Pond et al. [29] was to develop an experimentally-based mathematical and computational model of the skin to study the interplay of its material and structural properties and their evolution as a result of the intrinsic ageing process. Plausible mechanisms associated with ageing-induced material and microstructural evolution were explored in an attempt to explain observed effects associated with ageing (e.g. macroscopic stiffening of the tissue). This approach is presented in subesequent sections while a novel chemomechanobiological constitutive model of skin ageing that includes features of both intrinsic and extrinsic ageing is developed in Sec. 10.

\section{A summary of continuum mechanics}

The macroscopic response of skin to loading is mathematically described here using a continuum formulation. Prior to describing the model, the notation and key results from nonlinear continuum mechanics are summarised. For an extensive overview of continuum mechanics the reader is referred to [130], among others. 


\section{Notation}

Direct notation is adopted throughout. The scalar product of two vectors $\boldsymbol{a}$ and $\boldsymbol{b}$ is denoted by $\boldsymbol{a} \cdot \boldsymbol{b}$. The scalar product of two second-order tensors $\boldsymbol{A}$ and $\boldsymbol{B}$ is denoted by $\boldsymbol{A}: \boldsymbol{B}$. The composition of two second-order tensors $\boldsymbol{A}$ and $\boldsymbol{B}$ is denoted by $\boldsymbol{A} \boldsymbol{B}$. The action of a second-order tensor $\boldsymbol{A}$ on a vector $\boldsymbol{b}$ is a vector denoted by $\boldsymbol{A} \boldsymbol{b}$. The unit basis vectors in the Cartesian (standard-orthonormal) basis are $\left\{\boldsymbol{e}_{1}, \boldsymbol{e}_{2}, \boldsymbol{e}_{3}\right\}$. The dyad of two vectors $\boldsymbol{a}$ and $\boldsymbol{b}$ is a second-order tensor and is denoted by $\boldsymbol{a} \otimes \boldsymbol{b}$. Additional notation will be defined when introduced.

\subsection{Finite strain kinematics}

Consider a continuum body representing a portion of the skin. The reference configuration is defined as the placement of this body at time $t=0$, with that region denoted by $\Omega_{0}$ with boundary $\partial \Omega_{0}$ and outward unit normal $N$. As the body deforms, this region takes on subsequent configurations. At a current time $t$, the body occupies the region $\Omega$, referred to as the current configuration with boundary $\partial \Omega$ and outward unit normal $\boldsymbol{n}$. A motion $\boldsymbol{\chi}$ is assumed such that each material point $\boldsymbol{X} \in \Omega_{0}$ uniquely maps to a spatial point $\boldsymbol{x} \in \Omega$ at time $t$, i.e.

$$
\boldsymbol{x}=\boldsymbol{\chi}(\boldsymbol{X}, t) \quad \forall \boldsymbol{X} \in \Omega_{0} .
$$

The deformation of the body is characterised by the deformation gradient $\boldsymbol{F}$, defined by

$$
\boldsymbol{F}(\boldsymbol{X}, t):=\frac{\partial \boldsymbol{\chi}(\boldsymbol{X}, t)}{\partial \boldsymbol{X}}=\operatorname{Grad} \boldsymbol{\chi}(\boldsymbol{X}, t) .
$$

The determinant of $\boldsymbol{F}$ is defined by $J:=\operatorname{det} \boldsymbol{F}>0$. The right Cauchy-Green tensor $\boldsymbol{C}$, defined by

$$
\boldsymbol{C}:=\boldsymbol{F}^{T} \boldsymbol{F},
$$

provides a stretch measure in the reference configuration. Additionally, the principal scalar invariants of $\boldsymbol{C}$ are defined by

$$
I_{1}(\boldsymbol{C}):=\operatorname{tr}(\boldsymbol{C})=\boldsymbol{I}: \boldsymbol{C}, \quad I_{2}(\boldsymbol{C}):=\frac{1}{2}\left[\operatorname{tr}(\boldsymbol{C})^{2}-\operatorname{tr}\left(\boldsymbol{C}^{2}\right)\right], \quad \quad I_{3}(\boldsymbol{C}):=\operatorname{det}(\boldsymbol{C}) .
$$

Consider now the case of transverse isotropy where the material properties depend on a single given direction - a defining feature of skin. The preferred material direction at a point $\boldsymbol{X}$ is given by $\boldsymbol{v}_{0}$. The fabric tensor is then defined by

$$
\boldsymbol{A}_{0}:=\boldsymbol{v}_{0} \otimes \boldsymbol{v}_{0}
$$

and an additional invariant characteristic of transverse isotropic symmetry is given by

$$
I_{4}\left(\boldsymbol{C}, \boldsymbol{v}_{0}\right)=\boldsymbol{v}_{0} \cdot \boldsymbol{C} v_{0}=\lambda^{2},
$$

where $\lambda$ is the principal stretch along vector $v_{0}$ defined in the reference configuration.

\subsection{Constitutive relations for invariant-based transversely isotropic hyperelasticity}

A hyperelastic material is one for which a free energy $\psi$ acts as a potential for the stress. For homogeneous materials, the free energy is a function of the deformation gradient $\boldsymbol{F}$ and any additional tensor agency (e.g. the fabric tensor). 
As a general procedure to formulate constitutive equations for hyperelastic materials, one can postulate the existence of a free energy $\psi$, that is an isotropic function of the deformation. Hence, the first and second Piola-Kirchhoff stress tensors, $\boldsymbol{P}$ and $\boldsymbol{S}$, are defined by

$$
\boldsymbol{P}:=\frac{\partial \psi\left(\boldsymbol{F}, \boldsymbol{A}_{0}\right)}{\partial \boldsymbol{F}} \quad \text { and } \quad \boldsymbol{S}:=2 \frac{\partial \psi\left(\boldsymbol{C}, \boldsymbol{A}_{0}\right)}{\partial \boldsymbol{C}},
$$

where $\boldsymbol{P}=\boldsymbol{F S}$. The dependence of the free energy on $\boldsymbol{C}$ can be be expressed in terms of the $n_{\text {inv }}$ invariants of $\boldsymbol{C}$. The second Piola-Kirchhoff stress is thus given by

$$
\boldsymbol{S}=2 \sum_{i=1}^{n_{\text {inv }}} \frac{\partial \psi(\boldsymbol{C})}{\partial I_{i}} \frac{\partial I_{i}}{\partial \boldsymbol{C}}
$$

\subsection{Governing relations}

The balance of linear momentum, in the absence of inertial and body forces, and the natural boundary condition are given by

$$
\begin{array}{rr}
\operatorname{Div} \boldsymbol{P}=\mathbf{0} & \text { in } \Omega_{0}, \\
\boldsymbol{T}=\boldsymbol{P} \boldsymbol{N}=\overline{\boldsymbol{T}} & \text { on } \partial \Omega_{0, \mathrm{~N}},
\end{array}
$$

where Div is the material divergence operator. The Piola traction $\boldsymbol{T}$ is prescribed on the Neumann part of the boundary $\partial \Omega_{0, \mathrm{~N}} \subset \partial \Omega_{0}$. Dirichlet boundary conditions on the motion $\chi=\bar{\chi}$ are prescribed on $\partial \Omega_{0, \mathrm{D}}$ where $\partial \Omega_{0}=\partial \Omega_{0, \mathrm{D}} \cup$ $\partial \Omega_{0, \mathrm{~N}}$ and $\partial \Omega_{0, \mathrm{D}} \cap \partial \Omega_{0, \mathrm{~N}}=\emptyset$.

\subsection{Weak form of the governing relations}

The weak form of the governing equations and the accompanying Neumann boundary conditions in Eqs. (5) and (6) is essential for establishing the approximate solution using the finite element method (FEM). Multiplying the strong form of the equilibrium equation (5) by an vector-valued test function $\delta \boldsymbol{u}$, where $\delta \boldsymbol{u}=\mathbf{0}$ on $\partial \Omega_{0, \mathrm{D}}$, and applying the divergence theorem yields the expression for the weak form of the equilibrium equation as

$$
\int_{\Omega_{0}} \operatorname{Grad} \delta \boldsymbol{u}: \boldsymbol{P} \mathrm{d} V-\int_{\partial \Omega_{0, \mathrm{~N}}} \delta \boldsymbol{u} \cdot \overline{\boldsymbol{T}} \mathrm{d} A=0
$$

The weak form is the point of departure for the approximate solution of the equilibrium equation using the finite element method.

\section{A microstructurally-based constitutive model of skin}

Based on the experimental data summarised in this chapter, it is assumed that elastin and ground substance are the main contributors to the low modulus portion of the stress-strain curve which is largely linear and isotropic. The second region of the loading curve is dominated by the mechanical response of collagen fibres. As the collagen fibres straighten and take on load, they exponentially resist further stretch which results in a rapid nonlinear strain stiffening behaviour. This behaviour is also strongly anisotropic due the inherent preferred alignment of collagen fibres along the direction of extension. As the stiffening response of the skin is dominated by the response of the collagen network, 
the formulation of an effective microstructurally-motivated constitutive model is essential. Hence, it is postulated that the free energy $\Psi$ describing the overall mechanical behaviour of the dermis, assumed to be that of the skin because of the negligible contributions of the epidermis, is given by

$$
\Psi=\Psi_{\mathrm{gs}}+\Psi_{\text {elastin }}+\Psi_{\text {collagen }},
$$

where $\Psi_{\mathrm{gs}}, \Psi_{\text {elastin }}$ and $\Psi_{\text {collagen }}$ represent the free energy contributions from the ground substance, elastin and collagen, respectively.

To capture the behaviour of skin at low stretches, the elastin and ground substance free energies are given by the following compressible neo-Hookean type free energy:

$$
\Psi_{\mathrm{gs}}+\Psi_{\text {elastin }}=\left[\alpha_{\mathrm{gs}}+\alpha_{\text {elastin }}\right]\left[I_{1}-3+\frac{1}{\beta}\left[I_{3}^{-\beta}-1\right]\right]
$$

where

$$
\alpha_{(\bullet)}=\frac{\mu_{(\bullet)}}{2}, \quad \text { and } \quad \beta=\frac{v}{1-2 v}
$$

are constitutive parameters, respectively associated with the shear and volumetric response, and $(\bullet)$ is either "gs" or "elastin". The shear modulus is denoted by $\mu$, and $v$ is the Poisson's ratio of the composite material represented by the ground substance and elastin phases.

The nonlinear, anisotropic and network nature of the collagen response is captured through a transversely isotropic network eight-chain model proposed by Kuhl et al. [123]. This model is based on theories related to the micromechanics of macromolecule mechanical networks $[131,132]$ and is a particularisation of the orthotropic eight-chain model developed by Bischoff et al. [133]. In these mechanical descriptions of macromolecular networks, long molecular chains are assumed to rearrange their conformation under the influence of random thermal fluctuations (i.e. entropic forces). These type of idealised molecular chains are best described using the concept of wormlike chains by Kratky and Porod [134]. Wormlike chain models have been used to describe the structure and mechanical behaviour of collagen assemblies in the context of skin modelling [see e.g. 125, 133, 135-138] and other fibrous biological soft tissues [see e.g. 123, 124, 139, 140]. It is important to note that, in the development of microstructurally-based constitutive theories, these macromolecular chains could also be defined by or interpreted as either tropocollagen molecules, collagen micro-fibrils, fibrils, fibres or fibre bundles. If considering supra-molecular scales, it is clear that the wormlike chain energy is no longer associated with the notion of entropic elasticity and true molecular behaviour, but is rather a microstructurally-motivated macroscopic phenomenological energy that captures the strain-stiffening behaviour of collagenous structures. In the present approach, it is assumed that correlated chains represent collagen fibres (see Fig. 7).

The mechanics of macromolecular polymer structures is not only governed by the mechanical properties of individual chains but also by their electromagnetic and mechanical interactions which can take the form of covalent bonds, entanglement and physical cross-links. These combined effects give rise to strong isotropic and anisotropic network properties which can be implicitly and effectively captured by network models such as the eight-chain model of Arruda and Boyce [131] or Kuhl et al. [123]. The essential assumption underpinning these formulations is that there exists a representative microscopic unit cell able to capture network properties. The original eight-chain model of Arruda and Boyce [131] assumes that the unit cell is made of eight entropic chains of equal lengths connected from the centre of the cell to each of its corners (see Fig. 6), each equipped with their own entropic energy $\Psi_{\text {wormlike }}$. For correlated chains, the wormlike chain energy can be defined by

$$
\Psi_{\text {wormlike }}=\Psi_{0}+\frac{k \theta L}{4 A}\left[2 \frac{r^{2}}{L^{2}}+\frac{1}{\left[1-\frac{r}{L}\right]}-\frac{r}{L}\right]
$$

where $L, A, r_{0}$ and $r$ and are respectively the contour, persistence, initial end-to-end length and the current end-to-end length of the chain (see Fig. 7), and $\Psi_{0}$ is the wormlike chain energy in the unperturbed state. The wormlike chain has the defining characteristic that the chain segments are correlated and exhibit a smoothly-varying curvature along 


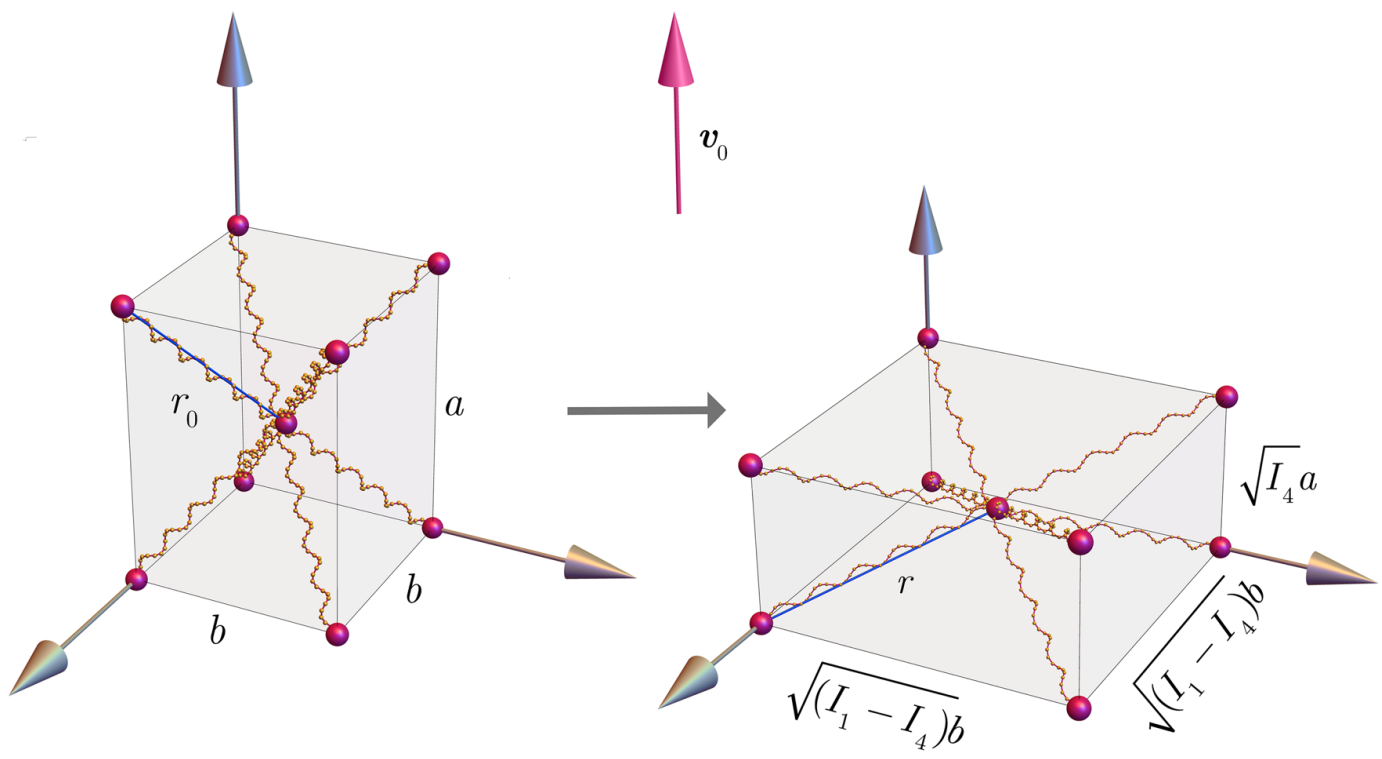

Fig. 6 Schematic representation of the transversely isotropic eight chain network model of Kuhl et al. [141]. The eight polymer chains are

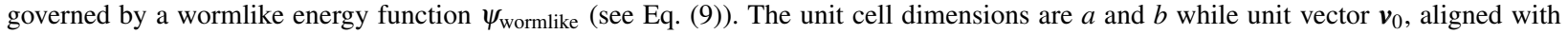
one of the principal direction of the unit cell, is the unit vector corresponding to the preferred orientation of the collagen network in the undeformed configuration [reproduced from 29].

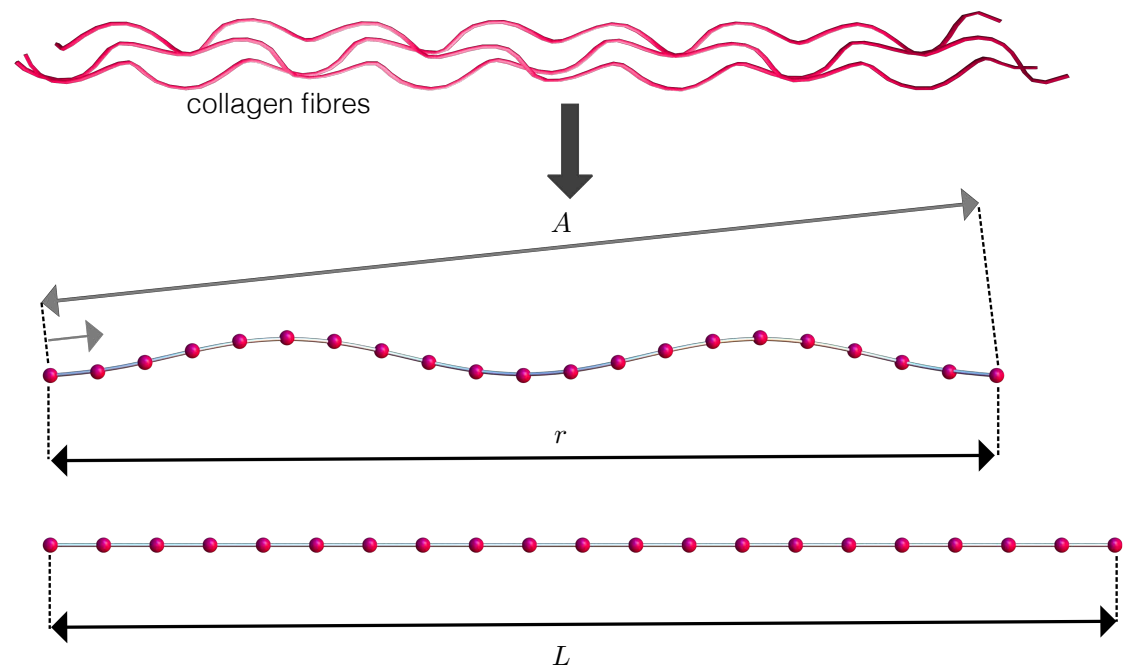

Fig. 7 Wormlike chain assembly. The successive chain link is correlated to the chain before it. $A$ is the persistence length, $r$ is the effective end-to-end length and $L$ is the chain contour length [reproduced from 29].

the contour. This correlated form is captured by the persistence length $A$. The persistence length can be viewed as a measure of stiffness. Garikipati et al. [125] refer to it as a measurement of the degree to which a chain departs from a straight line, while Marko and Siggia [142] interpret it as the characteristic length over which a bend can be made with energy $\operatorname{cost} k \theta$, where $k=1.380,648,52 \mathrm{~J} \mathrm{~K}^{-1}$ is the Boltzmann constant and $\theta$ the absolute temperature.

In order to incorporate such chain models into an invariant-based constitutive framework it is necessary to relate the individual chain stretch to the macroscopic deformation. To this end, the principle of affinity is invoked so that the macroscopic and unit cell principal directions are identical. Due to the symmetry of the chain structure, the stretch 
of each chain can be found as a function of the principle stretches. In Fig. 6, a unit cell arrangement of dimensions $a \times b \times b$ is depicted. For the case of anisotropy $a \neq b$. Additionally, the unit cell is characterised by the unit vector $\boldsymbol{v}_{0}$ that corresponds to the (local) preferred orientation of the collagen network. The undeformed end-to-end length of each of the individual chains is given by

$$
r_{0}=\frac{1}{2} \sqrt{a^{2}+2 b^{2}} .
$$

The deformed end-to-end length $r$ can be expressed using the invariants defined in Eq. (2) and Eq. (3) by

$$
r=\frac{1}{2} \sqrt{I_{4} a^{2}+\left[I_{1}-I_{4}\right] b^{2}} .
$$

The invariants $I_{1}, I_{3}$ and $I_{4}$ are measures of the macroscopic deformation of the polymer. It is assumed that the microscopic stretch along the direction $\boldsymbol{v}_{0}$ is captured by the macroscopic term $I_{4}$. This effectively couples the microscopic and macroscopic length scales. The relative stretch of a collagen fibre is defined by $\lambda_{\mathrm{r}}:=r / r_{0}$, see Eq. (9).

The final term of Eq. (7), characterising the contribution of collagen to the free energy, is further decomposed as

$$
\Psi_{\text {collagen }}=\Psi_{\text {chain }}+\Psi_{\text {repulsive }},
$$

where $\Psi_{\text {chain }}$ reflects the effective assembly of the eight chain energies, i.e. $\Psi_{\text {chain }}:=\gamma_{\text {chain }} \Psi_{\text {wormlike }}$ and $\Psi_{\text {repulsive }}$ is a repulsive energy that ensures the initial configuration is stress free and that the chain does not collapse. $\gamma_{\text {chain }}$ denotes the chain density per unit cell. Sáez et al. [140] interprets $\gamma_{\text {chain }}$ as the number of molecules within a collagen fibril, while Bischoff et al. [133] describes it as the collagen fibre density. In the context of collagen this measure corresponds to the number of fibres within a bundle. It follows that

$$
\begin{gathered}
\Psi_{\text {chain }}=\frac{\gamma_{\text {chain } k L \theta}}{4 A}\left[2 \frac{r^{2}}{L^{2}}+\frac{1}{1-\frac{r}{L}}-\frac{r}{L}\right], \\
\Psi_{\text {repulsion }}=-\frac{\gamma_{\text {chain }} k \theta}{4 A}\left[\frac{1}{L}+\frac{1}{4 r_{0}} \frac{1}{1-\frac{r_{0}}{L}}-\frac{1}{4 r_{0}}\right]\left[\ln \left[I_{4}^{\left[a^{2}-b^{2}\right] / 2}\right]+\frac{3}{2} \ln \left[I_{1}^{b^{2}}\right]\right] .
\end{gathered}
$$

All stretch quantities have been normalised by the link length $L$ which is the length of a single link along the wormlike chain assembly.

A description of the constitutive parameters of $\Psi_{\text {collagen }}$ is summarised in Table 1 . The expression for the first Piola-Kirchhoff stress $\boldsymbol{P}$ then follows directly from Eq. (4).

\begin{tabular}{lll}
\hline Parameter & \multicolumn{2}{l}{ Symbol Units } \\
\hline Poisson's ratio & $v$ & - \\
Shear modulus & $\mu$ & $\mathrm{N} \mathrm{m}^{-2}$ \\
Boltzmann constant & $k$ & $\mathrm{~J} \mathrm{~K}^{-1}$ \\
Absolute temperature & $\theta$ & $\mathrm{K}$ \\
Chain density & $\gamma_{\text {chain }}$ & $\mathrm{m}^{3}$ \\
Contour length & $L$ & - \\
Persistence length & $A$ & - \\
Unit cell dimensions & $a, b$ & - \\
\hline
\end{tabular}

Table 1 Constiutive parameters of $\Psi_{\text {collagen }}$.

\section{Microstructurally-based mechanistic model indirectly accounting for intrinsic ageing}

A central hypothesis underpinning this study is that the effects of ageing (both intrinsic and extrinsic) on the mechanical properties of the tissue are directly linked to alterations in the microstructural characteristics of the collagen and 
elastin networks. In this section, some of the constitutive parameters introduced in Sec. 7 are motivated as plausible mechanistic descriptions of the intrinsic ageing process as evidenced by experimental observations (see Table 2). It is worth emphasising that, in reality, the material and structural effects of ageing are likely to be coupled and would therefore lead to a dependency between constitutive parameters. For the sake of simplicity, and in the absence of relevant experimental data, this interplay is not accounted for. In addition, the effect of intrinsic ageing on the ground substance manifests itself as a more pronounced viscoelastic response with age. As the constitutive model for the ground substance currently only captures the elastic response it is assumed that ageing does not affect the ground substance related parameters in Eq. (8).

\begin{tabular}{lll}
\hline Constituent & Ageing effect & Parameter \\
\hline Ground substance & Increased water content results in more pronounced viscoelasticity & $\mathrm{n} / \mathrm{a}$ \\
\hline Elastin & Loss of elasticity & $\alpha_{\text {elastin }}$ \\
& Destruction of elastin network & $\alpha_{\text {elastin }}$ \\
\hline \multirow{2}{*}{ Collagen } & Loss of mature collagen & $\gamma_{\text {chain }}$ \\
& Increased crosslinking & $\gamma_{\text {chain }}$ \\
& Flattening and unravelling of collagen network & $a, b$ \\
& Alterations in anisotropy & $a, b$ \\
\hline
\end{tabular}

Table 2 Description of the constitutive parameters, the evolution of which is associated with ageing.

The elastin network is observed to degrade with age. The loss of elastic integrity would logically lead to a reduced contribution from the elastin component to the composite strain energy function (7). One approach could be to introduce a volume fraction for elastin, ground substance and collagen. Degradation of the elastin network would then be modelled as a reduction in volume fraction of elastin. Instead, here, the value of $\alpha_{\text {elastin }}$ is assumed to decrease with age. It should be noted again that elastin is a highly-stable protein which undergoes little turnover before the age of 40. Thus $\alpha_{\text {elastin }}$ would remain constant for the first four decades.

Within the current model, there is no natural parameter that explicitly describes the level of collagen crosslinking at any point in time. It is believed that the increased crosslinking with age is one factor responsible for the increased stiffness of the skin. Through increasing $\gamma_{\text {chain }}$, it is expected that a stiffer response will be elicited. Although $\gamma_{\text {chain }}$ describes the number of fibres within a bundle, it represents the closest link to the observed increase in crosslinking with age. It would be expected that a decrease in collagen density would contribute to a decrease in the overall stiffness of the skin as the collagen network loses integrity. Thus it is reasonable to assume that a decrease in $\gamma_{\text {chain }}$ with age could describe a loss of collagen.

As discussed previously, the slope of the linear region of the stress-strain curve for skin, as a composite structure, remains constant with age but the onset of strain hardening occurs at lower strains. This suggests that there might be a mechanism that simply alters the structural characteristics of the collagen network and not the mechanical properties of collagen microfibrils. In their unloaded states collagen fibres are crimped. It is believed that this conformational state is due to the presence of highly cross-linked elastic fibres - mainly elastin fibres [143] — and is also the result of active tensions exerted by fibroblasts. Any reduction in fibroblast density in the collagen dermal network would have an effect on these active forces and on the rest state of collagen fibres. Such a reduction would tend to relax the residual strain/tension in the dermis. This would make collagen fibres less crimped and therefore their apparent length would be closer to their fully-taut length. Thus, when the skin is macroscopically loaded in tension, the dermal tissue will not exhibit a very pronounced toe region - corresponding to the progressive uncrimping of collagen fibres but will rather reach the stiffer linear region more quickly. The corollary of this observation is that for a given applied macroscopic strain, the response of the intrinsically-aged dermal collagen network will be stiffer. With age, due to dermal flattening and the loss of elastic recoil, the collagen fibres are observed to uncrimp [16]. In the chain network structure of the model, this is captured by adjustments to the end-to-end length of the collagen fibres through the parameters $a$ and $b$. By increasing $a$ and $b$ there will be a reduction in the range of strain of the low modulus portion prior to the onset of the collagen response at lower stretches. Thus by increasing $a$ and $b$ the model should be capable of capturing the stiffening response. Additionally, the anisotropic response of the skin is observed to change with age. Tonge et al. [144] reports a loss of anisotropy in experiments using a bulge test. As the model developed here will be 
compared to the bulge test results at various ages, it is this behaviour that is intended to be captured. By reducing the ratio of $a / b$, either by increasing $b$ or decreasing $a$ or a combination of both, this should be achieved.

Undeniably, there is a reduction in the thickness of the skin with age (see Eq. (1)). Such an observation is backed by numerous sources in the literature $[39,55,57]$, but there is still much debate over the exact relationship between age and skin thinning. The changes in the dermal thickness with age will be captured directly in the geometry of the computational model.

\section{A microstructurally-based model of skin intrinsic ageing with age-dependent constitutive parameters}

The objective is to now identify how the subset of constitutive parameters assumed to be associated with intrinsic ageing, indetified in the previous section, can be determined at specific times in the ageing cycle using a combined experimental and computational approach.

\subsection{Parameter identification}

Multiple mechanical skin tests have been proposed to characterise the skin response to loading. The variety of skin tests and different methods to perform them, as well as the natural skin variation that exists through factors such as ethnicity, gender, age and anatomical site, have resulted in a broad and varying characterisation of skin behaviour. In general, in vivo, in both physiological and supra-physiological conditions, the skin exhibits material nonlinearity, anisotropy, viscoelasticity and near incompressibility and can also sustain large deformations [119]. However, elicitation and relevance of these characteristics is highly variable. Although there are many experimental techniques to characterise certain aspects of skin mechanics [120], bulge tests are considered here. Performed in vitro, the bulge test applies a positive pressure to the underside of an excised skin sample. The skin sample is fixed at a specified diameter aperture which allows for "bulge-like" deformation of the sample under pressure. Deformations are measured and linked to the state of stress so that constitutive parameters can be identified [41, 144]. The choice of the bulge experiment as a test-bed for the constitutive and computational and models developed here is motivated by the following factors:

- As the experiments are performed in vitro, the boundary conditions of the numerical model are simpler to define and impose.

- The large deformations induced in the physical tests ensure that the various components of the constitutive model are activated. At small deformations the elastin and ground substance will assume the dominant role, and ultimately, as macroscopic strains increase, the collagen fibres will become active and dictate the majority of the response at large deformations.

- The specimens were taken from the back of the patients, thus minimising any photoageing effects such as those observed on sun-exposed regions (e.g. the face).

- The experimental results published by Tonge et al. [41, 144] provide a broad range of kinematic measurements with which to compare the models.

- Crucially, the experiments by Tonge et al. [41, 144] were conducted on skin samples harvested from donors featuring a broad range of ages. This allows the correlation between age and mechanical properties (i.e. constitutive parameters) to be studied.

In the bulge test follows performed by Tonge et al., several $10 \times 10 \mathrm{~cm}$ skin specimens were procured from the back torso of donors, ages ranging from 43 to 83 years. After excision, the adipose tissue was removed and tissue thickness measured. The thickness, gender, age and anatomical site of the samples are listed in Table 3. Thickness was measured at the middle of each edge of the sample and the average taken. This average was used as the uniform thickness of the sample in the numerical model.

The specimens were glued to a $7.5 \mathrm{~cm}$ diameter ring. The ring serves to constrain the skin specimen. The skin interior to the ring is subject to pressure loading while that exterior is fully constrained. The coordinate system for the 


\begin{tabular}{|c|c|c|c|}
\hline \multicolumn{3}{|c|}{ Age Gender Site } & \multirow{2}{*}{$\begin{array}{l}\text { Thickness [mm] } \\
4.86\end{array}$} \\
\hline 43 & Male & Lower back & \\
\hline 44 & Male & Lower back & 4.38 \\
\hline 59 & Female & Unknown & 5.18 \\
\hline 61 & Male & Left upper back & 2.01 \\
\hline 62 & Female & Unknown & 2.95 \\
\hline 83 & Male & Unknown & 2.43 \\
\hline
\end{tabular}

Table 3 Donor and specimen information from Tonge et al. [41].

samples was set such that the $y$-axis corresponded to the vertical body axis and the $x$-axis to the horizontal body axis, as shown in Fig. 8. Fibre and perpendicular directions can thus be defined by the angle $\Phi$ from the horizontal axis. Controlling for relative humidity and temperature, samples were inflated through a controlled applied pressure, with a maximum pressure of $5.516 \mathrm{kPa}$. Upon inflation the skin samples deformed to an elliptical dome. The dimensions of the ellipse are used to determine the dominant fibre direction with $\Phi$ defined accordingly.

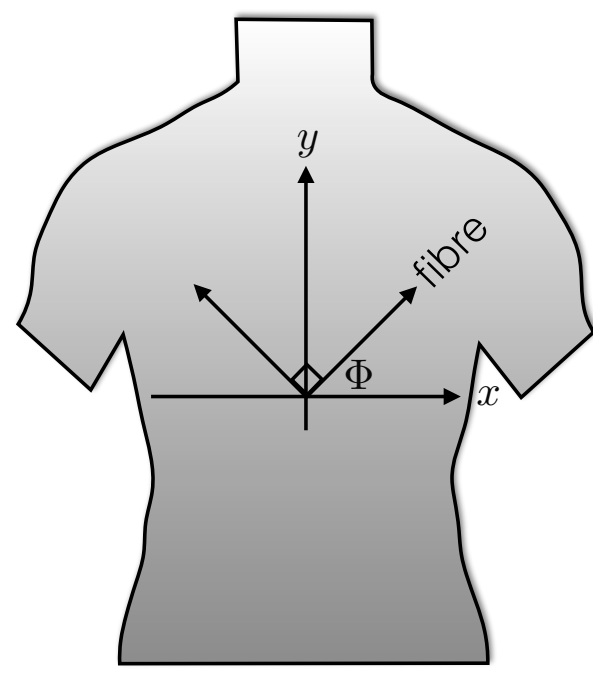

Fig. 8 Body axes: the $y$-axis corresponds to the vertical body axis and the $x$-axis to the horizontal body axis. Recreated from [144].

A finite element updating technique [see e.g. 120,145, 146] is used to identify the constitutive parameters of the transversely isotropic eight-chain model of the skin for various ages. Fig. 9 shows the discretisation of the skin sample. The skin thickness is set to the dermal thicknesses as specified in Table 3. The geometry is discretised using 8-noded hexahedral trilinear elements, with 5 elements through the thickness. Through a mesh sensitivity study, it was found that 46,416 nodes ensured a sufficiently converged solution. The nodes on the upper surface outside the boundary ring and the outer edges are fully constrained. The pressure loading condition is applied to the bottom surface. As proposed in the previous section, only the original network dimensions $a$ and $b, \gamma_{\text {chain }}$ and $\alpha_{\text {elastin }}$ are assumed to be variable. The rest of the parameters are assumed age invariant and are given by $L=2.125, A=1.82, \gamma_{\mathrm{gs}}=100 \mathrm{~Pa}, \beta=4.5$, $\theta=310 \mathrm{~K}$. Furthermore, only the tests for male specimens are simulated in order to exclude the influence of variations between genders.

In Fig. 10 the profile of the skin obtained from the finite element simulation of the bulge test on the 44 year-old male specimen is given at monotonically-increasing pressure loading from $34.37 \mathrm{~Pa}$ to $5.52 \mathrm{kPa}$. The profiles are given along and perpendicular to the fibre direction $\boldsymbol{v}_{0}$ as illustrated by the arrows in each subfigure. The bulge specimen undergoes a rapid initial displacement while the stress state is still within the low modulus portion of the stress-strain curve. This can be seen in the plot of the pressure versus the in-plane stretch where a small increment in pressure 


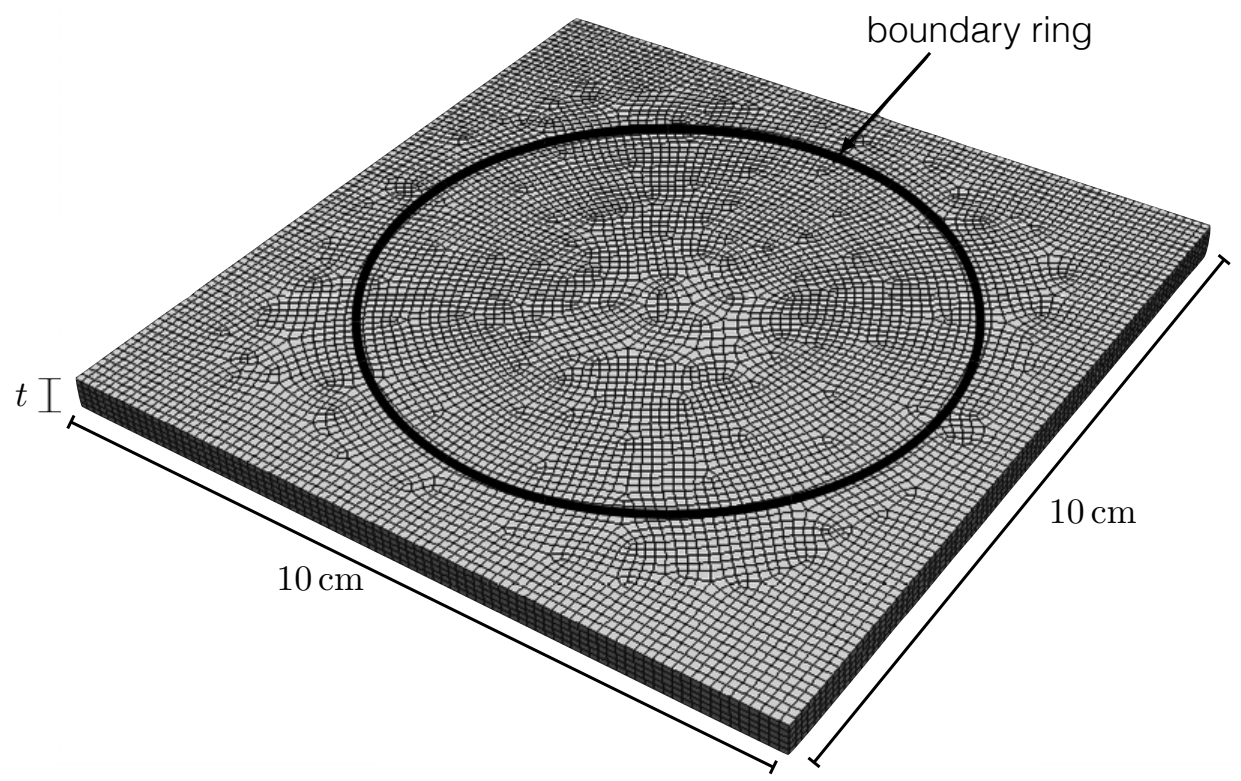

Fig. 9 Finite element mesh used for the bulge tests [adapted from 29].

induces a large change in stretch (Fig. 11). During this phase, the elastin and ground substance contributions dominate the mechanical response and offer little resistance to inflation. The divergence of the pressure-stretch lines indicates the activation of the anisotropic collagen network. In terms of the material model, the collagen energy contribution is no longer negligible as the end-to-end chain length $r$ undergoes significant stretch and approaches the contour length $L$. For the age 44 specimen, further stretch parallel to the fibre direction above $400 \mathrm{~Pa}$ does not occurs whereas perpendicular stretch continues to increase with pressure. This results in the response shown in Fig. 10(c) where at $502 \mathrm{~Pa}$ the profiles along the perpendicular and parallel directions differ. Mechanical anisotropy is amplified with increased pressure as the perpendicular stretch increases. As the specimen is deformed, exponentially more pressure is needed to attain further deformation which is characterised by the locking-type limits. In Fig. 10(d) and Fig. 10(e) displacement at high pressure occurs laterally in the perpendicular fibre direction.

The 8-chain model is now used to simulate the stretch behaviour of the skin at increasing pressure for increasing age, and the results summarised in Fig. 11. The stretch is measured parallel and perpendicular to the dominant fibre direction. In general, the stretch behaviour with increasing age is reasonably captured. The fit at age 43 is notably poor which suggests that there is an additional directional dependence not captured by the model or a missing constituent contribution (i.e. an incomplete constitutive model), or experimental error.

Excluding the response of the age 43 test, the identified parameters shown in Table 4 are in agreement with the hypotheses advocated in Sec. 8. Note, $\gamma_{\text {chain }}$ does not vary monotonically with age, with the same values obtained for age 44 and 83 . This suggests that $\gamma_{\text {chain }}$ is not age dependent. The difference in the initial end-to-end lengths as dictated by the increased values of $a$ and $b$ allows for the difference in maximum stretches observed in the experiments. Additionally the ratio between the two changes with age with $a: b=4.313: 1,2.84: 1,2.836: 1$ at age 44, 61 and 83 respectively. This reduction, especially between age 44 and 61 , is indicative of the expected reduction in anisotropy. The value of $\alpha_{\text {elastin }}$ decreases almost linearly with age, with an approximate reduction of $100 \mathrm{Nm}^{-2}$ every five years. 


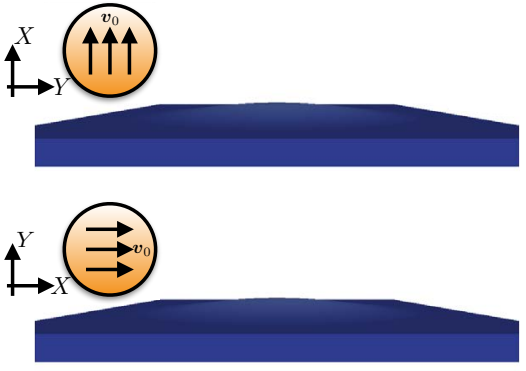

(a) $34.37 \mathrm{~Pa}$

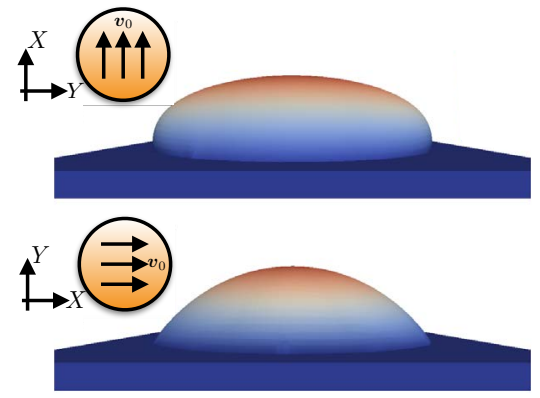

(d) $1840 \mathrm{~Pa}$

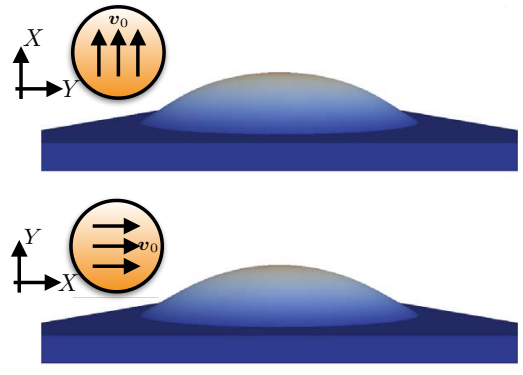

(b) $206.4 \mathrm{~Pa}$

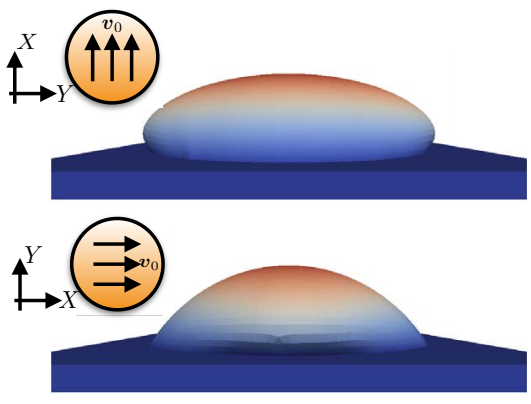

(e) $5520 \mathrm{~Pa}$

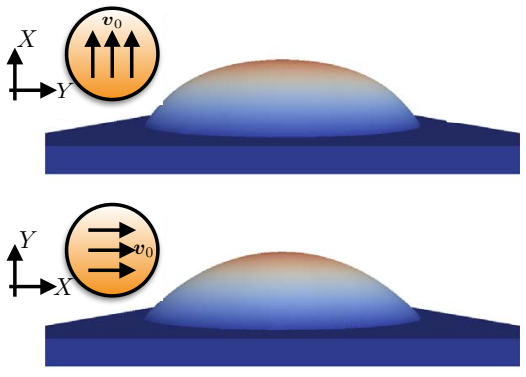

(c) $502.1 \mathrm{~Pa}$

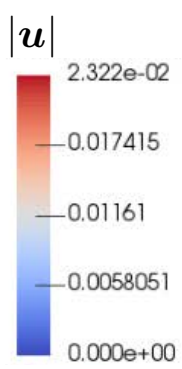

0.000 e+00

Fig. 10 Displacement profiles for the 44 years-old male skin specimen: (a) pressure $=34.37 \mathrm{~Pa}$; (b) pressure $=206.4 \mathrm{~Pa}$; (c) pressure $=$ $502.1 \mathrm{~Pa}$; (d) pressure $=1840 \mathrm{~Pa}$; (e) pressure $=5520 \mathrm{~Pa}$ [reproduced from 29].

\begin{tabular}{lllll}
\hline Parameter & Age 43 & Age 44 & Age 61 & Age 83 \\
\hline$a$ & 3.58 & 3.45 & 3.55 & 3.65 \\
$b$ & 0.5 & 0.8 & 1.25 & 1.287 \\
$\gamma_{\text {chain }}\left(\times 10^{22}\right)$ & $6 \mathrm{~m}^{-3}$ & $0.856 \mathrm{~m}^{-3}$ & $0.0856 \mathrm{~m}^{-3}$ & $0.856 \mathrm{~m}^{-3}$ \\
$\alpha_{\text {elastin }}$ & $1000 \mathrm{Nm}^{-2}$ & $1300 \mathrm{Nm}^{-2}$ & $1000 \mathrm{Nm}^{-2}$ & $500 \mathrm{Nm}^{-2}$ \\
\hline
\end{tabular}

Table 4 Wormlike 8-chain model parameter values for the age 43, 44, 61 and 83 bulge test.

\subsection{Evolution of the parameters in the microstructurally-based mechanistic model with intrinsic ageing}

The factors discussed in the previous sections provide a means by which the skin constitutive model (and its associated finite element model replicating bulge tests) can capture ageing effects in a continuous sense through the modification of a few selected parameters. Using the $a$ and $b$ parameter values as found from the age 44,61 and 83 fits, general ageing trends were established. It is clear that a mere three data points is insufficient in order to conclusively determine an ageing trend, but they are adequate for the current proof of concept. Shape-preserving fitting algorithms were used to find a continuous evolution of $a$ and $b$ as shown in Fig. 12(a) and Fig. 12(b). As expected, there is a general increase in both parameters with age: $a$ evolves almost linearly with age whereas $b$ undergoes a large increase between age 44 and 61 followed by a plateau. This suggests that between age 44 and 61 there is a more significant loss of anisotropy due to a realignment or redistribution of the collagen fibres as a network as compared to later in life.

The skin thickness data as presented in Table 3 was used to determine a linear equation for the skin thickness as a function of age. From Fig. 12(c) it can observed that a very rough linear fit can been established as

$$
t=-0.0047[\mathrm{~mm} / \text { year }] \times \text { age }[\text { years }]+0.59[\mathrm{~mm}] \text {. }
$$




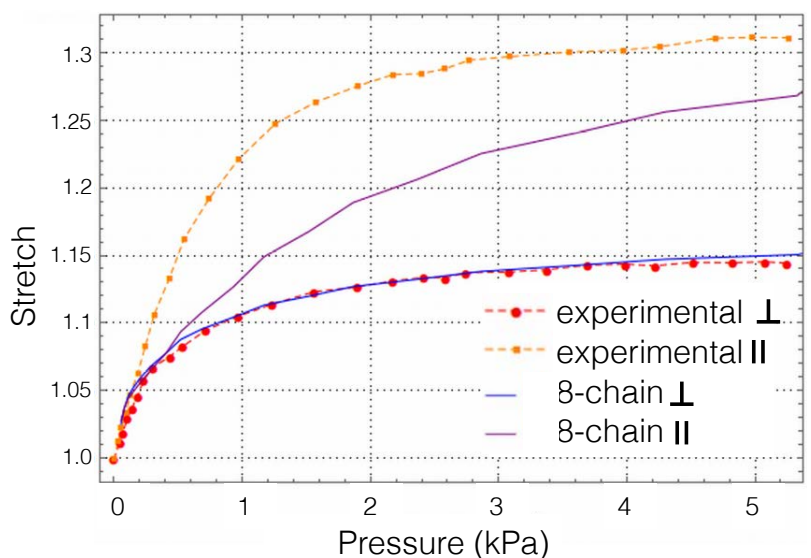

a) age 43

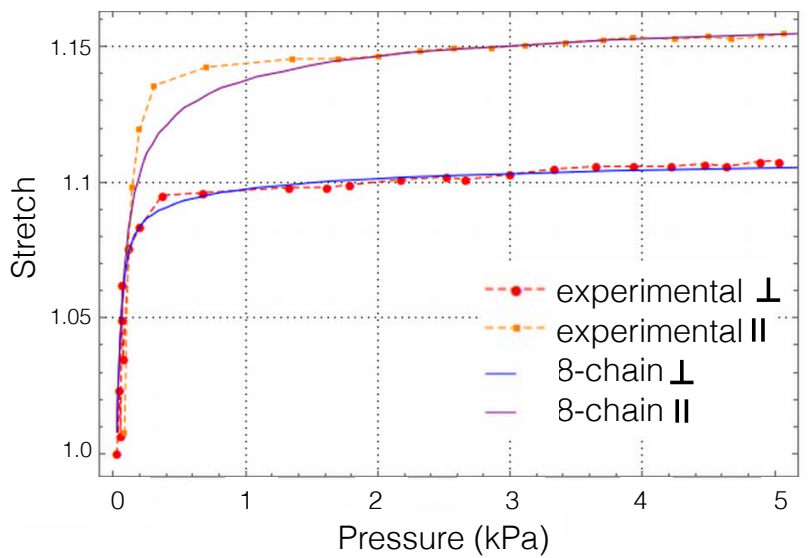

(b) age 61

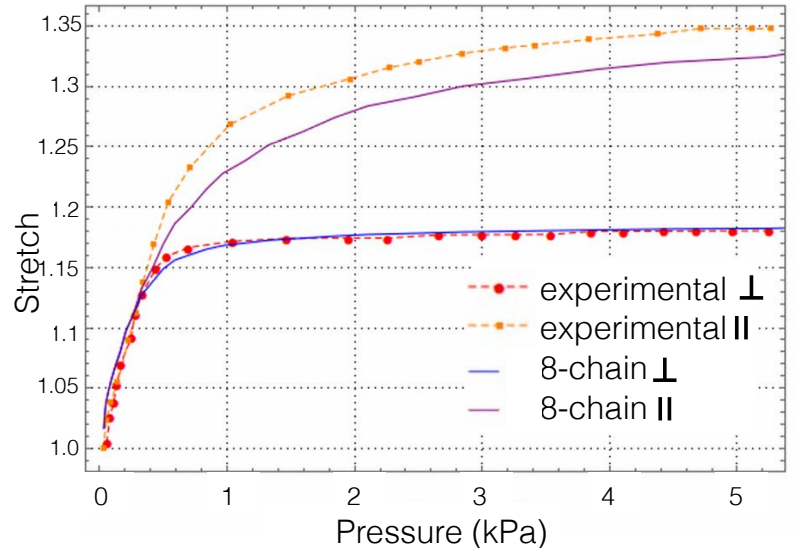

b) age 44

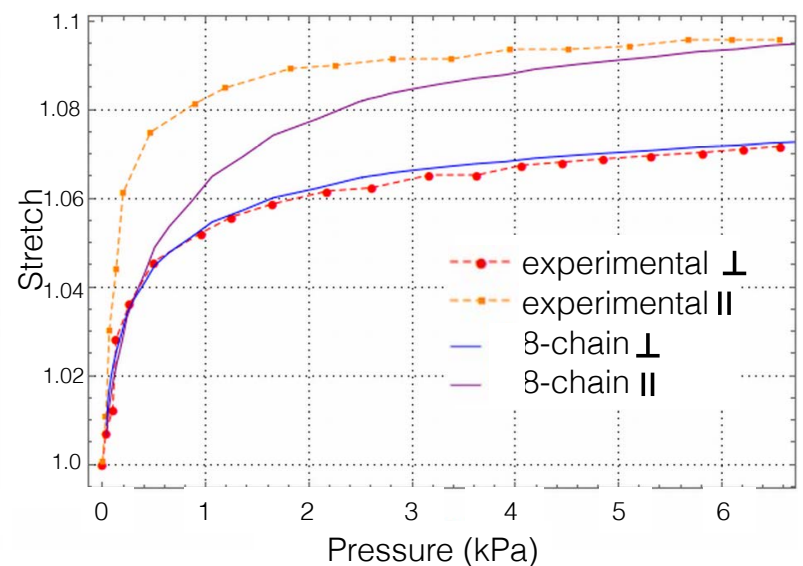

(d) age 83

Fig. 11 Plots of in-plane stretch as a function of inflation pressure for the experimental measurements of Tonge et al. [144] overlaid over the theoretical curves obtained from the identification of constitutive parameters [reproduced from 29].

This should be compared with Eq. (1). The overall decrease in skin thickness is consistent with experimental values found in the literature.

With the established trends, bulge test simulations were run at 5 year increments. Table 5 contains the adjustments to the parameters of interest. As mentioned, $\alpha_{\text {elastin }}$ decreases from $1300 \mathrm{~N} / \mathrm{m}^{2}$ at age 44 by $100 \mathrm{~N} / \mathrm{m}^{2}$ every 5 years.

\begin{tabular}{lccc}
\hline Age & $a$ & $b$ & $\alpha_{\text {elastin }}$ \\
\hline 49 & 3.483 & 0.984 & 1200 \\
54 & 3.513 & 1.14 & 1100 \\
59 & 3.54 & 1.236 & 1000 \\
64 & 3.564 & 1.26 & 900 \\
69 & 3.587 & 1.273 & 800 \\
74 & 3.613 & 1.282 & 700 \\
79 & 3.634 & 1.287 & 600 \\
84 & 3.654 & 1.288 & 500 \\
89 & 3.671 & 1.285 & 400
\end{tabular}

Table 5 Modification of age dependent model parameters with intrinsic ageing. 

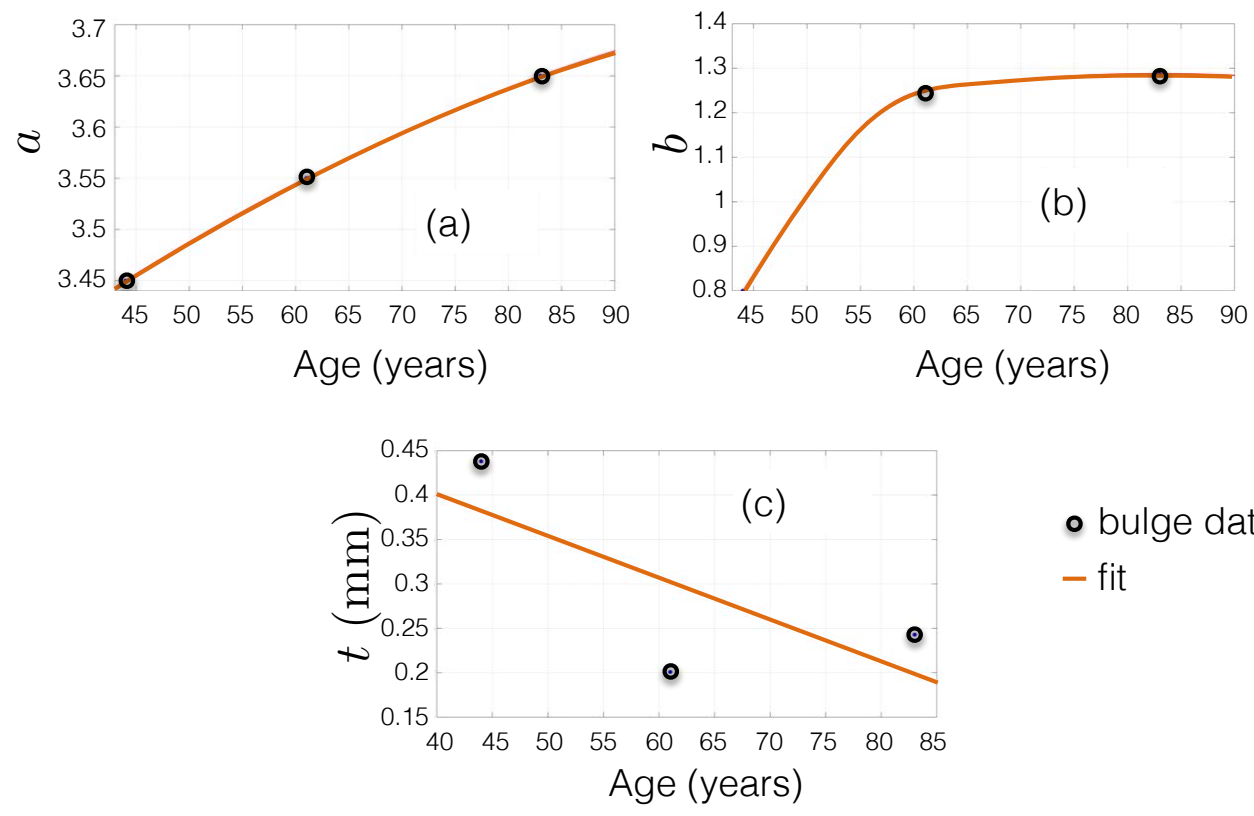

- bulge data

- fit

Fig. 12 Network dimension and skin thickness trends with age as found through bulge test fits [reproduced from 29].

In Fig. 13 the simulated evolution of the stretch behaviour with age is given, and compared to the experimental data at ages 44,61 and 83. As expected, the general ageing-induced trend is captured as the skin stiffens with age. The maximum stretch obtained drops significantly between ages 44 and 59, with less significant drops thereafter. This is to be expected primarily due to the trend in parameter $b$ as given in Fig. 13(b), where there is an initial sharp increase followed by a plateau. The convergence of the stretch values between ages 59 and 89 is due to the initial end-to-end length $r_{0}$ approaching the contour length $L$. As $r_{0}$ increases with age, the amount of allowable macroscopic stretch is reduced.

(a) II

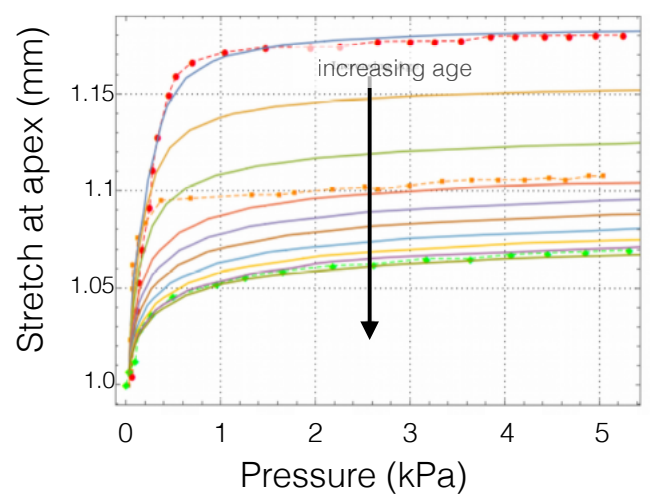

(b) $\perp$

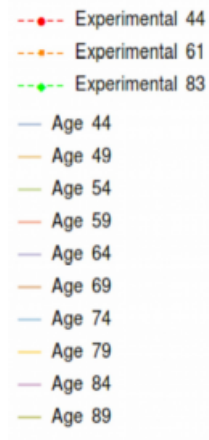

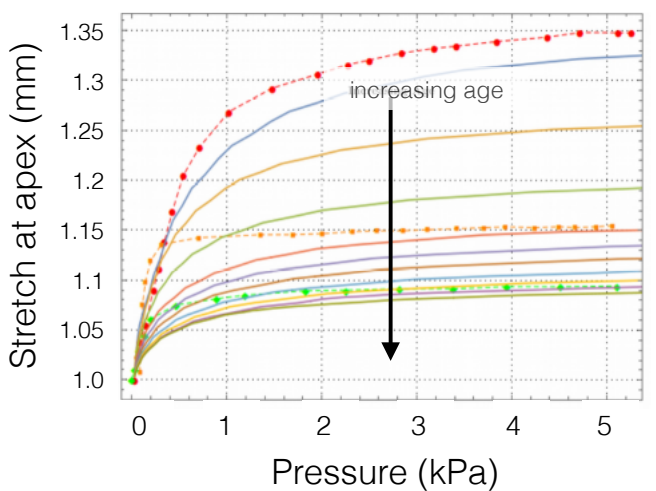

Fig. 13 Stretch at the apex of the pressurised skin as a function of applied pressure for 9 age values (see Table 5); (a) parallel to the fibre direction; (b) normal to the fibre direction [reproduced from 29]. 
The contour plots of the displacement components for the age 44 specimen are displayed in Fig. 14(I), with the fibre direction along the $x$-axis. A comparison is given with the experimental results obtained by Tonge et al. [144]. As expected, the contour profiles for each displacement component are comparable and the overall behaviour of the simulations matches the experimental results. The age 44 simulations accurately capture the displacement along the $x$-axis (see Fig. 14(Ia)), but the displacements along the $y$ - and $z$-axes, shown in Fig. 14(Ib-Ic), are slightly overestimated by approximately $0.5 \mathrm{~mm}$ and $2 \mathrm{~mm}$, respectively. This overestimation arises from a constituent contribution not captured in the model. It is also possible that the effect of the rig set-up is not appropriately captured in the simulation. The guard ring may introduce a compressive force through the thickness that would limit the extent of the deformation.

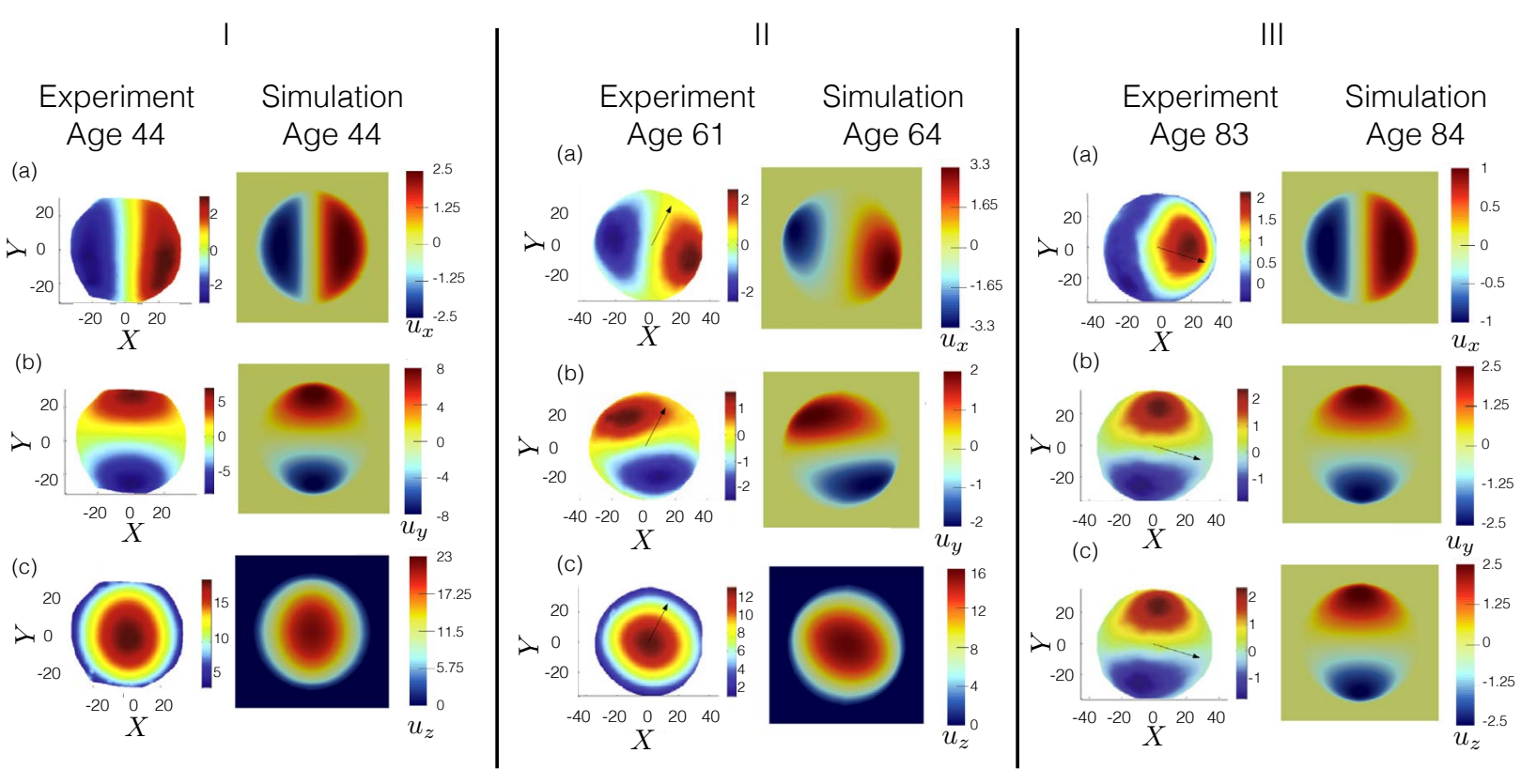

Fig. 14 Plots of deformed profile for the bulge test coloured by the various componenets of the displacement [mm]. The simulations are compared to the experimental results of Tonge et al. [144] for various different ages (I-III) [reproduced from 29].

Fig. 14(II-III) shows the simulated contour profile of the bulge test for samples according to the proposed "ageing" of the various parameters. These profiles are compared to experimental results at approximately the same age, as extracted from the data obtained by Tonge et al. [144]. It is important to note that in the experimental specimens the Langer lines were not aligned with the body axes. The Langer lines were oriented at $64^{\circ}$ and $-24.6^{\circ}$ degrees to the $x$-axis for the age 61 and 83 specimens, respectively, as indicated in the figures. This alteration in the orientation is reflected in the simulated results.

The profile comparison between the age 64 simulated and age 61 experimental results are very similar to those of age 44 . The alteration in the fibre orientation is captured quite satisfactorily, with the resulting axial assymmetry in the components of displacement along the $x$ - and $y$-axes represented accurately. The $z$ component of displacement in the out-of-plane direction is acceptably captured, although there seems to be an overestimation in the level of anisotropy in the simulated results. In terms of the magnitude of the displacements, the $y$-axis components of the age 61 experiment and age 64 simulation are similar, but the simulations overestimate the other two components. This is not too surprising considering the unsatisfactory fit with the pressure-stretch data at age 61 as shown in Fig. 13.

The age 84 simulations were orientated at $-24.6^{\circ}$ to the positive $x$-axis in order to replicate the age 83 experimental contour plots. There is sufficient agreement in the general profile of the simulated results when compared to the experimental data. The only discrepancy lies in the component along the $x$-axis of the experimental data as shown in Fig. 14(IIIa), where the lack of symmetry suggests a possible defect in the skin specimen, such as a non-uniform skin thickness or an irregularity on a constituent level, such as an inconsistent collagen distribution or dispersion. Despite 
this, the displacements along the $x$ - and $y$-axes of the age 84 simulated results are comparable to the experimental results, but there is again a minor overestimation in the $z$-axis component.

\section{A microstructurally-based chemo-mechanobiological model of skin ageing}

A microstructurally-based model that attempts to capture the contribution of key constituents on the overall mechanical response of the skin has been presented in Sec. 7. An ageing type response was introduced in Sec. 9 by extending the mechanical model via the modification of parameters motivated to be involved. The proposed ageing model did not however incorporate the mechanisms directly involved in the turnover of collagen and elastin that occur during the ageing processes.

One extension to the skin model is now presented as a possible framework to describe a continuous ageing response. Evolution equations for the nano-constituents and their influence on collagen and elastin are proposed. With reference to Fig. 5, the focus here will be on chronological ageing, while further ideas will be given for an extension that incorporates photoageing.

\subsection{Biological remodelling}

Continuum models that account for changes in the volume and constitutive properties of biological materials — key features of ageing — can be broadly classified as:

- Continuum models for growth, i.e. a change in volume [see e.g. 141, 147, 148].

- Continuum models for remodelling, i.e. a change in constituent density and constitutive parameters.

A remodelling approach to ageing is proposed here. Continuum models for remodelling were originally developed for hard tissue (bone) using the framework of continuum damage mechanics [see e.g. 149, 150]. These models have been extended to describe softening behaviour in soft biological tissue [see e.g. 151]. Evolution equations for the density field and the elasticity tensor follow from thermodynamic arguments.

An alternative remodelling framework not based on damage mechanics is open-system thermodynamics. This allows for isotropic remodelling via the introduction of density sources and fluxes [141, 148, 152]. The free energy $\Psi$ has added dependence on the density thereby allowing for strengthening or weakening of the material microstructure. Anisotropic remodelling has been considered by Waffenschmidt et al. [153]. The general structure for remodelling is described by two coupled governing relations: one for the momentum balance (see Eq. (5)) and one for mass conservation. This framework has been successfully employed to different biological systems undergoing remodelling and more recently to soft tissue. Of particular relevance is the work on turnover in arteries by Sáez et al. [140], Lafortune and Aris [154]. The work by Sáez et al. is especially useful here in the context of skin ageing, as a theoretical and computational model for collagen turnover as a result of hypertension is proposed. The model focused on the variation of the nano-constituent factors, such as TIMP, MMP and TGF- $\beta$. This variation is driven by mechanical stimuli as a result of the muscular contractions. The remodelling framework of Sáez et al. thus provides a basis for an ageing model. The framework is now discussed in more detail prior to developing the ageing model for skin.

Hypertension is a chronic vascular disease where blood pressure becomes elevated. The increased pressure results in an increased mechanical load on the vasculature. In an attempt to maintain a homoeostatic stress state, smooth muscle cells (SMCs) start to grow. This causes thickening of the vessel walls. Thickening is the result of alterations within the extracellular matrix (ECM) of vessel walls, primarily through changes in the deposition and degradation of collagen.

Extensive studies have shown that collagen deposition increases with hypertension. SMCs produce TGF- $\beta$ and due to the added mechanical stress on the SMCs, there is increased expression of TGF- $\beta$. This results in the up-regulation of collagen deposition. 
In terms of degradation of collagen, the ratio of MMP to TIMP is of primary concern. In hypertension, TIMP has been reported to increase, decreasing the total amount of MMP. Ultimately, collagen turnover increases as a result of hypertension and the mechanical loading imposed on the SMCs.

A key aspect of the model is how the collagen density $\rho$ evolves. Density evolution introduces a mass source term $\mathscr{R}$ to the balance of mass. As proposed by Kuhl et al. [123], $\mathscr{R}$ takes the general form:

$$
\dot{\rho}=\mathscr{R}:=\left[\frac{\rho}{\rho^{*}}\right]^{-m} \Psi-\Psi^{*},
$$

where $\Psi^{*}$ and $\rho^{*}$ are the energy in the homoeostatic equilibrium state (also know as the attractor stimulus) and the initial density, respectively, and $m$ is a parameter.

Additionally, $\Psi_{\text {col }}$ takes on a density dependence, $\Psi_{\mathrm{col}}=\Psi_{\mathrm{col}}\left(\mathbf{C}, \mathbf{v}_{0}, \rho\right)$ similar to that in Eq. (12). This either strengthens or weakens the collagen contribution to the overall mechanical response according to the presence of collagen within the soft tissue.

Through activation of the SMCs, TGF- $\beta$ and TIMP pathways are activated. It is these constituents that evolve according to Eq. (12). As mentioned, the system is driven by the mechanical loading on the SMCs which is captured through an energy, $\Psi_{S M C}$ and source terms for TGF and TIMP given by

$$
\begin{aligned}
\mathscr{R}_{\mathrm{TGF}-\beta} & =\gamma_{\mathrm{TGF}-\beta}\left[\frac{\rho_{\mathrm{TGF}-\beta}}{\rho_{\mathrm{TGF}-\beta}^{*}}\right]^{-m_{\mathrm{TGF}-\beta}} \Psi_{\mathrm{SMC}}-\Psi_{\mathrm{TGF}-\beta}^{*}, \\
\mathscr{R}_{\mathrm{TIMP}} & =\gamma_{\mathrm{TIMP}}\left[\frac{\rho_{\mathrm{TIMP}}}{\rho_{\mathrm{TIMP}}^{*}}\right]^{-m_{\mathrm{TIMP}}} \Psi_{\mathrm{SMC}}-\Psi_{\mathrm{TIMP}}^{*},
\end{aligned}
$$

where $\gamma \in \mathbb{R}^{+}$quantify the relative contribution to the SMC energy.

TIMP acts to inhibit and regulate MMP. Thus MMP evolves inversely to TIMP as

$$
\mathscr{R}_{\mathrm{MMP}}=\gamma_{\mathrm{MMP}} \mathscr{R}_{\mathrm{TIMP}}
$$

where $\gamma_{\text {MMP }} \in \mathbb{R}^{-}$.

With the collagen sources defined, collagen evolves as

$$
\mathscr{R}_{\mathrm{col}}=\gamma_{+} \mathscr{R}_{\mathrm{TGF}-\beta}+\gamma_{-} \mathscr{R}_{\mathrm{MMP}},
$$

where $\gamma_{+}$and $\gamma_{-}$characterise the sensitivity of collagen to TGF- $\beta$ and MMP respectively.

The above system of time-dependent nonlinear equations can be solved using a time discretisation scheme and linearised through a Newton scheme to establish the collagen density evolution. The parameter units and interpretation are summarised in Table 6.

\begin{tabular}{llc}
\hline Parameter & \multicolumn{1}{c}{ Symbol } & Units \\
\hline TGF- $\beta$ sensitivity to SMC energy & $\gamma_{\text {TGF- }} \beta$ & - \\
TIMP sensitivity to SMC energy & $\gamma_{\text {TIMP }}$ & - \\
MMP sensitivity to TIMP & $\gamma_{\text {MMP }}$ & - \\
Collagen sensitivity to TGF- $\beta$ & $\gamma_{+}$ & - \\
Collagen sensitivity to MMP & $\gamma_{-}$ & - \\
$m_{\text {TGF- } \beta}$ & TGF- $\beta$ nonlinear remodelling & - \\
$m_{\text {TIMP }}$ & TIMP nonlinear remodelling & - \\
$\rho_{\text {TIMP }}^{*}$ & initial TIMP density & $\mu \mathrm{g} \mathrm{ml}^{-1}$ \\
$\rho_{\text {TGF- } \beta}^{*}$ & initial TGF- $\beta$ density & $\mu \mathrm{gl}^{-1}$ \\
$\rho_{\text {MMP }}^{*}$ & initial MMP density & $\mu \mathrm{gl}^{-1}$ \\
$\rho_{\text {col }}^{*}$ & initial collagen density & $\mu \mathrm{g} \mathrm{ml}^{-1}$ \\
$\Psi_{\text {TGF- } \beta}^{*}$ & homoeostatic equilibrium state of TGF- $\beta$ & $\mathrm{Nm}^{-2}$ \\
$\Psi_{\text {TIMP }}^{*}$ & homoeostatic equilibrium state of TIMP & $\mathrm{Nm}^{-2}$ \\
\hline
\end{tabular}

Table 6 Evolution equation parameters for collagen turnover in hypertension [140]. 


\subsection{A model for skin ageing}

The model of Sáez et al. [140] provides a framework that can be extended to account for collagen and elastin remodelling due to intrinsic and extrinsic ageing. The interplay between factors such as TGF- $\beta$ and MMP are critical for the ageing model. The primary difference between the model presented by Sáez et al. and an ageing model is how the processes are driven. In Sáez et al., the stimulus is related to the elastic energy density where changes in collagen turnover occur due to the mechanical loading of the SMCs of the vascular tissue. This offers a very natural driving process captured by the SMC free energy $\Psi_{\mathrm{SMC}}$. The ageing process does not offer such an easy analogue.

Ageing, and accordingly the effects on collagen and elastin turnover, do not evolve with mechanical loading but instead evolve due to a more innate process. Additionally, in the Sáez et al. model the system is driven toward a state of equilibrium through the inclusion of the homoeostatic equilibrium terms, $\Psi_{\mathrm{TGF}-\beta}^{*}$ and $\Psi_{\mathrm{TIMP}}^{*}$. Within an ageing context, it does not make sense for the system to be driven to a state of equilibrium as this would be analogous to saying that ageing stops. Due to these differences, and in order to develop a meaningful ageing model, several adjustments need to be made.

A primary challenge is the introduction of an alternative driving factor. In Sec. 4.3, the link between the loss of antioxidant defence with age and the increase in the the level of ROS was described. Accordingly ROS was found to be the common factor in the evolution of the primary constituents that related to collagen and elastin turnover (see Fig. 5). Therefore it is proposed that ROS provides a mechanism with which to drive the ageing process.

With reference to Eq. (12), the evolution equations are postulated to be of the form

$$
\dot{\rho}^{\mathrm{rel}}=\mathscr{R},
$$

where $\mathscr{R}$ represents the source term as before. As no initial values could be found in the literature for all the constituents that play a role in collagen and elastin turnover in the skin, relative quantities $\rho^{\text {rel }}$ will be used to describe the constituent evolution, where the relative quantities are the current values normalised by the value at time $t=0$ denoted by $\rho^{*}$. Hence

$$
\rho^{\mathrm{rel}}=\frac{\rho}{\rho^{*}} .
$$

This normalisation further avoids any dimensional and scale issues. Accurate initial values are also not of concern (especially as this is an exercise in model development) as the relative initial value is always unity.

The proposed ageing model is now assessed by its ability to replicate key processes depicted in Fig. 5, with ROS as the independent variable driving the evolution equations. The primary factors that are dependent on ROS are TGF- $\beta$ and TIMP. TGF- $\beta$ levels have been found to increase with ROS accumulation in the extracellular matrix, while TIMP levels show a decline with ROS accumulation within the fibroblast cells. Thus their respective proposed source terms are given by

$$
\begin{aligned}
\mathscr{R}_{\mathrm{TGF}} & =\frac{\gamma_{\mathrm{TGF}}^{+}}{\tau_{\mathrm{TGF}}^{+}}\left[\frac{\rho_{\mathrm{TGF}}^{\max }-\rho_{T G F}^{\mathrm{rel}}}{\rho_{\mathrm{TGF}}^{\max }-1}\right]^{n_{\mathrm{TGF}}}\left[\rho_{\mathrm{ROS}}^{\mathrm{rel}}-1\right], \\
\mathscr{R}_{\mathrm{TIMP}} & =\frac{\gamma_{\mathrm{TIMP}}^{-}}{\tau_{\mathrm{TGF}}^{-}}\left[\frac{\rho_{\mathrm{TIMP}}^{\mathrm{rel}}-\rho_{\mathrm{TIMP}}^{\min }}{1-\rho_{\mathrm{TIMP}}^{\min }}\right]^{m_{\mathrm{TIMP}}}\left[\rho_{\mathrm{ROS}}^{\text {rel }}-1\right] .
\end{aligned}
$$

Additionally, through ROS and TIMP evolution, MMP levels will be affected. In order to simplify and reduce the number of the equations, the dependence on AP-1 as a governing factor in MMP turnover is absorbed into the dependence on ROS, giving a source term for MMP as

$$
\mathscr{R}_{\mathrm{MMP}}=\frac{\gamma_{\mathrm{MMP}}^{-}}{\tau_{\mathrm{MMP}}^{-}}\left[\frac{\rho_{\mathrm{MMP}}^{\max }-\rho_{\mathrm{MMP}}^{\mathrm{rel}}}{\rho_{\mathrm{MMP}}^{\max }-1}\right]^{m_{\mathrm{MMP}}}\left[\rho_{\mathrm{TIMP}}^{\mathrm{rel}}-1\right]+\frac{\gamma_{\mathrm{MMP}}^{+}}{\tau_{\mathrm{MMP}}^{+}}\left[\frac{\rho_{\mathrm{MMP}}^{\max }-\rho_{\mathrm{MMP}}^{\mathrm{rel}}}{\rho_{M M P}^{\max }-1}\right]^{n_{\mathrm{MMP}}}\left[\rho_{\mathrm{ROS}}^{\mathrm{rel}}-1\right] .
$$

Thus, the relative collagen and elastin mass density sources are given by 


$$
\begin{aligned}
& \mathscr{R}_{\mathrm{col}}=\frac{\gamma_{\mathrm{col}}^{-}}{\tau_{\mathrm{col}}^{-}}\left[\frac{\rho_{\mathrm{col}}^{\mathrm{rel}}-\rho_{\mathrm{col}}^{\mathrm{min}}}{1-\rho_{\mathrm{col}}^{\min }}\right]^{m_{\mathrm{col}}}\left[\rho_{\mathrm{MMP}}^{\mathrm{rel}}-1\right]+\frac{\gamma_{\mathrm{col}}^{+}}{\tau_{\mathrm{col}}^{+}}\left[\frac{\rho_{\mathrm{col}}^{\mathrm{max}}-\rho_{\mathrm{col}}^{\mathrm{rel}}}{\rho_{\mathrm{col}}^{\max }-1}\right]^{n_{\mathrm{col}}}\left[\rho_{\mathrm{TGF}}^{\mathrm{rel}}-1\right], \\
& \mathscr{R}_{\text {elas }}=\frac{\gamma_{\text {elas }}^{-}}{\tau_{\text {elas }}^{-}}\left[\frac{\rho_{\text {elas }}^{\text {rel }}-\rho_{\text {ela }}^{\text {min }}}{1-\rho_{\text {elas }}^{\text {min }}}\right]^{m_{\text {elas }}}\left[\rho_{\mathrm{MMP}}^{\text {rel }}-1\right]+\frac{\gamma_{\text {elas }}^{+}}{\tau_{\text {elas }}^{+}}\left[\frac{\rho_{\text {elas }}^{\text {max }}-\rho_{\text {elas }}}{\rho_{\text {elas }}^{\max }-1}\right]^{n_{\text {elas }}}\left[\rho_{\mathrm{TGF}}^{\text {rel }}-1\right] .
\end{aligned}
$$

In order to capture the reduction in collagen sensitivity to TGF- $\beta$, a ROS-dependent modification to $\gamma_{\text {col }}^{+}$is proposed as

$$
\mathscr{R}_{\gamma_{\mathrm{col}}^{+}}=\frac{\gamma_{\gamma_{\mathrm{col}}}^{-}}{\tau_{\gamma_{\mathrm{col}}}^{-}}\left[\rho_{\mathrm{ROS}}^{\mathrm{rel}}-1\right]
$$

The various model parameters are summarised in Table 7. $\tau$ is introduced as an adaptation speed parameter to allow for control over the reaction rate of one constituent relative to another, as well as to ensure dimensional consistency.

\begin{tabular}{llc}
\hline Parameter & \multicolumn{1}{c}{ Symbol } & Units \\
\hline TGF- $\beta$ sensitivity to ROS & $\gamma_{\text {TGF- } \beta}^{+}$ & - \\
TIMP sensitivity to ROS & $\gamma_{\text {TIMP }}^{-}$ & - \\
MMP sensitivity to TIMP & $\gamma_{\text {MMP }}^{-}$ & - \\
MMP sensitivity to ROS & $\gamma_{\text {MMP }}^{+}$ & - \\
Collagen sensitivity to TGF- $\beta$ & $\gamma_{\text {col }}^{+}$ & - \\
Collagen sensitivity to MMP & $\gamma_{\text {col }}^{-}$ & - \\
Elastin sensitivity to TGF- $\beta$ & $\gamma_{\text {elas }}^{-1}$ & - \\
Elastin sensitivity to MMP & $\gamma_{\text {elas }}^{-}$ & - \\
$m, n$ & Remodelling factors & - \\
$\tau$ & Adaptation speed & years \\
$\rho^{\max }$ & Constituent relative maximum & - \\
$\rho^{\min }$ & Constituent relative minimum & - \\
\hline
\end{tabular}

Table 7 Evolution equation parameters.

In the literature, it has been consistently reported that the features common to chronological ageing begin to manifest at around 40 years of age. Within the first four decades, ROS levels are relatively stable, where ROS production is balanced through oxidative defence. Thereafter, due to decline in the oxidative defence capabilities of the skin, ROS levels have been shown to gradually increase, with levels up to 4 times greater in aged skin as compared to young skin. As a simple model, a relative ROS evolution is proposed as depicted in Fig. 15 and governed by

$$
\rho_{\mathrm{ROS}}^{\mathrm{rel}}=\left\{\begin{array}{ll}
1 & t \leq 35 \\
0.1(t-35)+1 & t>35
\end{array} .\right.
$$

That is, ROS is chosen to remain stable until age 35 , whereafter it increases linearly with age.

The chosen parameter values are given in Table 8. All $\tau$ values were set to 1 year and the remodelling factors $m$ and $n$ to 0.8 . Choices for the relative minimum and maximum values of the various constituents were determined from the literature discussed in Sec. 4. Where values could not be found, reasonable values were estimated to replicate the expected constituent response. In the absence of evolution data from actual experiments with which to calibrate the model, the proposed sensitivity parameters merely provide a proof of concept.

In Fig. 16, the simulated evolution of TGF- $\beta$, TIMP and MMP are shown. Within the first simulated 35 years, there is no change in the relative amounts of each factor. Thereafter, there is a gradual decline in the level of TIMP, while MMP and TGF- $\beta$ increase. In Fig. 16d, the sensitivity of collagen to TGF- $\beta$ is shown to reduce due to a decline in $\gamma_{\text {col }}^{+}$.

Through the dependence on MMP and TGF- $\beta$, collagen and elastin levels decline as depicted in Fig. 17a and Fig. 17b, respectively. As discussed previously, collagen has been shown to decline with age to approximately half the amount when compared to younger skin. As captured in Fig. 17a, despite the increase in TGF- $\beta$, the combination of increased MMP and reduced TGF- $\beta$ sensitivity results in the relative amount of collagen declining towards the proposed relative minimum. As the relative minimum of 0.5 is approached, the rate of collagen degradation decreases. 


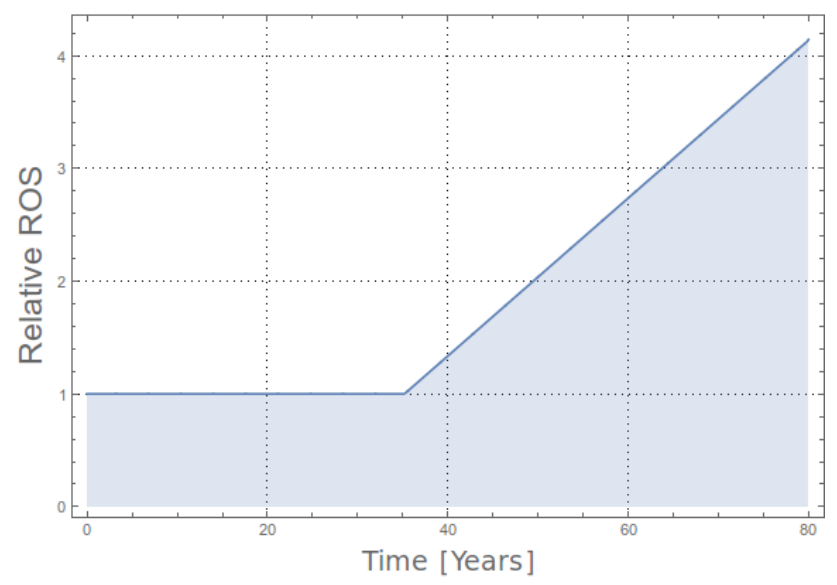

Fig. 15 Relative ROS evolution with time.

\begin{tabular}{ll}
\hline Parameter & \multicolumn{1}{c}{ Value } \\
\hline$\gamma_{T G F-\beta}^{+}$ & 0.005 \\
$\gamma_{T I M P}^{-}$ & -0.005 \\
$\gamma_{M M P}^{-}$ & -0.02 \\
$\gamma_{M M P}^{+}$ & 0.1 \\
$\gamma_{\text {col }}^{+}$ & 0.005 \\
$\gamma_{\text {col }}^{-}$ & -0.02 \\
$\gamma_{\text {elas }}^{+}$ & 0.005 \\
$\gamma_{\text {elas }}^{-}$ & -0.01 \\
$\gamma_{\text {col }}^{-}$ & -0.00003 \\
$\rho_{T G F}^{\max }-\beta$ & 4 \\
$\rho_{\text {TIMP }}^{\min }$ & 0.7 \\
$\rho_{\text {MMP }}^{\max }$ & 4 \\
$\rho_{\text {col }}^{\max }$ & 1.5 \\
$\rho_{\text {col }}^{\text {min }}$ & 0.5 \\
$\rho_{\text {elas }}^{\max }$ & 3 \\
$\rho_{\text {elas }}^{\min }$ & 0.8 \\
\hline
\end{tabular}

Table 8 Evolution equation parameter values.

The evolution of elastin mimics that of collagen, although due to the inherently stable nature of elastin, the relative loss of elastin is not as severe.

Fig. 16 - 17 provide an example of the evolution profile of the various constituents that have been identified to play a role in the ageing process. With this, it is essential to link the evolution of collagen and elastin back to the mechanical model. In order to accomplish this, inspiration is taken from that of the theory of constrained mixtures.

In the constrained mixture theory adopted here, the evolving constituent composition and organisation are innately linked to the overall mechanical behaviour of the soft tissue. The primary idea behind a constrained mixture formulation is to pay attention to the individual constituents within a tissue by accounting for the turnover rates of the constituents of interest, rather than focusing on the overall change of the tissue. For a detailed overview, the reader is referred to [155-159].

Denoting $\rho^{\mathrm{k}}(s)$ as the mass density of constituent $\mathrm{k}$ at time $s$, the free energy is proposed to take the form

$$
\Psi=\Psi\left(\mathbf{F}, \rho^{\mathrm{k}}(s)\right),
$$

where any anisotropic behaviour has been omitted for simplicity. This structure of the free energy follows the general rule of mixtures where weighted contributions are used to predict the mean response of a composite material. That is, 


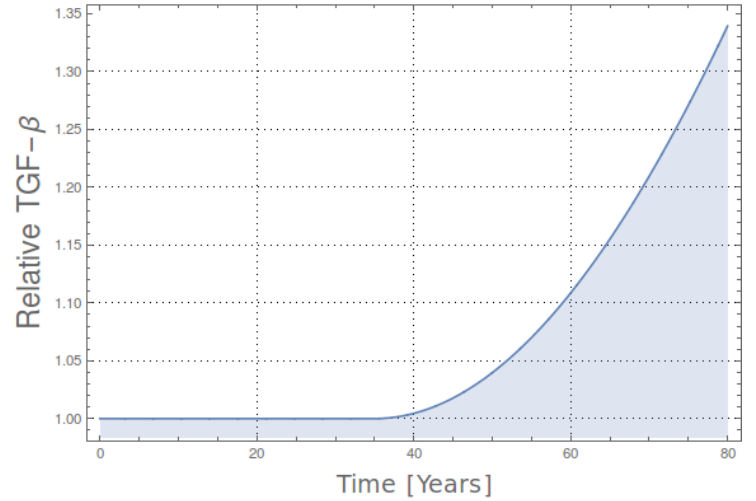

(a) Simulated TGF- $\beta$ evolution with time.

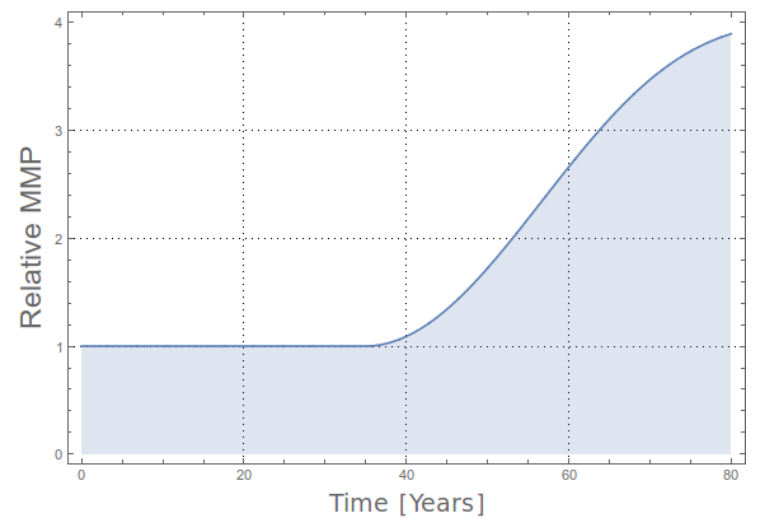

(c) Simulated MMP evolution with time.

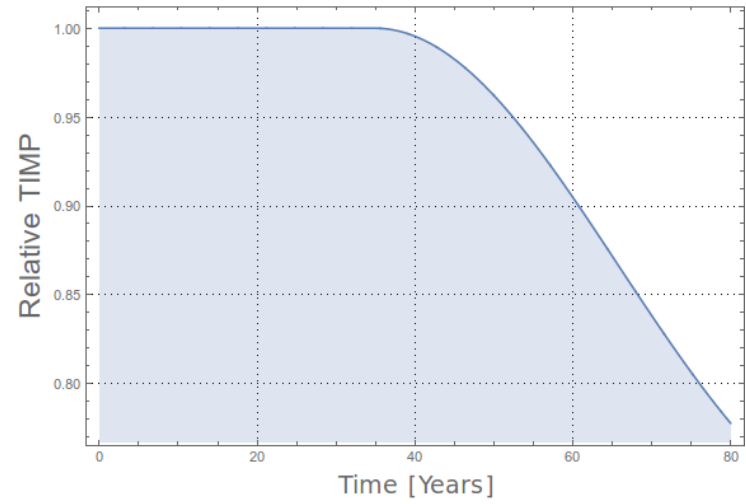

(b) Simulated TIMP evolution with time.

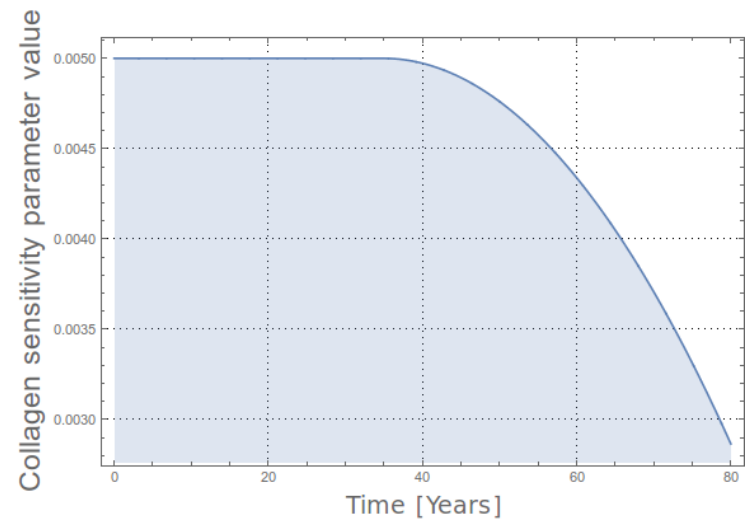

(d) Simulated $\gamma_{\text {col }}^{+}$evolution with time.

Fig. 16 Evolution of nano-constituents TGF- $\beta$, TIMP and MMP according to evolution equations.

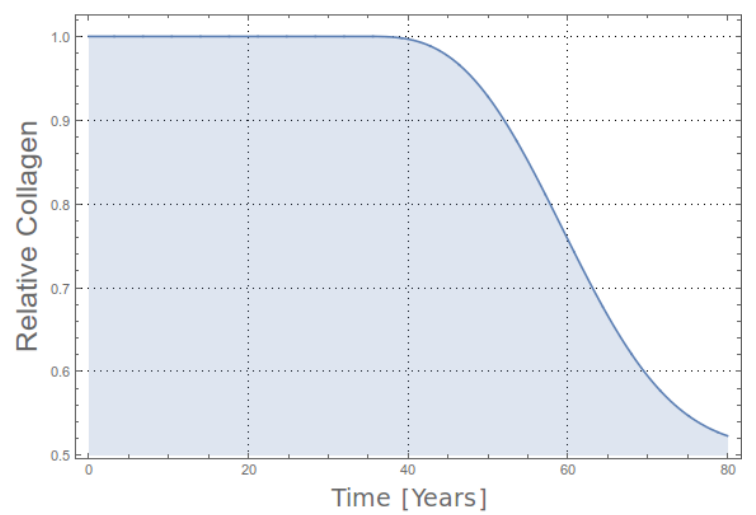

(a) Evolution of collagen with time.

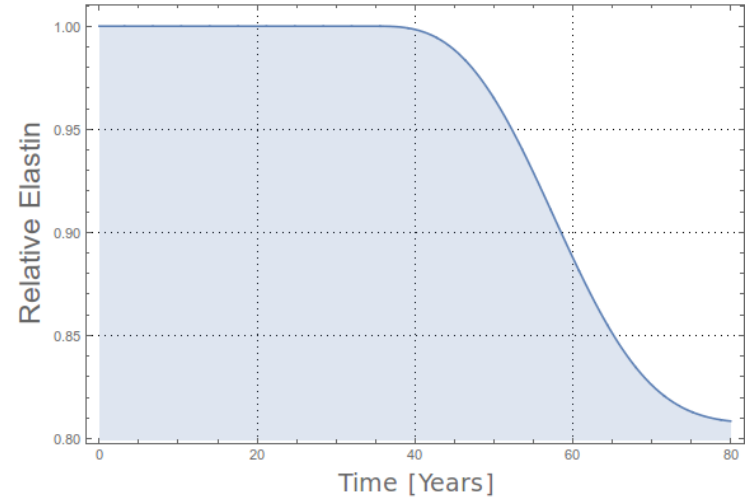

(b) Evolution of elastin with time.

Fig. 17 Simulation evolution of collagen and elastin with time

$$
\Psi\left(\mathbf{F}, \rho^{\mathrm{k}}(s)\right)=\sum_{k} \frac{\rho^{\mathrm{k}}(s)}{\rho(s)} \Psi^{\mathrm{k}}
$$


where $\rho(s)=\sum_{k} \rho^{\mathrm{k}}(s)$ and $\Psi^{\mathrm{k}}$ is the free energy contribution of constituent k. $\rho(s)$ is generally assumed to be constant [157]. Each constituent mass density $\rho^{\mathrm{k}}(s)$ can thus be allowed to adapt in time according to some stimulus. Evolution equations for each constituent can be proposed, in a manner similar to that proposed for the contituent evolutions. Thus, as an extension to Eq. (7), each constituent free energy is weighted by the corresponding relative mass density (Eq. (13)), that is

$$
\Psi=\rho_{\mathrm{gs}}^{\mathrm{rel}} \Psi_{\mathrm{gs}}+\rho_{\mathrm{elas}}^{\mathrm{rel}} \Psi_{\text {elas }}+\rho_{\mathrm{col}}^{\mathrm{rel}} \Psi_{\mathrm{col}} .
$$

The choice of using relative density weightings is motivated by the idea that in the absence of constituent remodelling, Eq. (17) reduces to Eq. (7). Eq. (14) and Eq. (15) can be used as evolution equations for the relative collagen and elastin contributions, respectively, while $\rho_{\mathrm{gs}}^{\text {rel }}$ evolves to ensure the overall density remains constant.

As mentioned, the model as presented in Eq. (7) does not have a natural connection to the amount of collagen and elastin present in the skin. This mixture-theory motivated extension addresses this issue. Additionally, with the form of the collagen free energy as presented in Eq. (10), this allows $\gamma_{\text {chn }}$ to be a representation of the level of crosslinking within the collagen network.

\subsection{A model for photoageing}

In Sec. 4.3 photoageing was presented as a processes that not only compounds the effects of chronological ageing, but often induces structural changes more severe than those observed under normal ageing. Due to this, the extension of the mixture-type model to a one that accounts for photoageing is the logical next step.

From Fig. 5, the processes involved in chronological and photoageing are very similar, which makes the extension to account for photoageing a very natural one. The primary difference is the formation of what is referred to as elastotic tissue. This is similar to elastic tissue but highly-disorganised and irregular. Thus a simple extension of the free energy (17) would be

$$
\Psi=\rho_{\mathrm{gs}}^{\mathrm{rel}} \Psi_{\mathrm{gs}}+\rho_{\mathrm{elas}}^{\mathrm{rel}} \Psi_{\text {elas }}+\rho_{\mathrm{col}}^{\mathrm{rel}} \Psi_{\mathrm{col}}+\rho_{\text {elastotic }}^{\mathrm{rel}} \Psi_{\text {elastotic }},
$$

where $\Psi_{\text {elastotic }}$ is an elastotic free energy. The form of $\Psi_{\text {elastotic }}$ could be similar to that presented for the elastin free energy $\Psi_{\text {elas }}$, but experimental data would be needed to make an informed decision. The evolution process would similarly be driven by ROS generation, where ROS would take on a form such as

$$
\mathrm{ROS}=\mathrm{ROS}_{\text {chrono }}+\mathrm{ROS}_{\mathrm{UV}} .
$$

Here $\mathrm{ROS}_{\text {chrono }}$ and $\mathrm{ROS}_{\mathrm{UV}}$ are the ROS generated by the chronological and UV-exposure, respectively. $\mathrm{ROS}_{\text {chrono }}$ would take on a form as proposed in Eq. (16) whereas ROS $_{U V}$ would be motivated by actual UVA data obtained from measurementx.

The evolution equations for TGF- $\beta$, TIMP and MMP would accordingly need to include terms dependent on ROS $_{\mathrm{UV}}$. Additionally, an evolution equation for elastotic tissue would need to be proposed which would be very similar to that of the elastin evolution equation (15). Through such a framework, photoageing could be accounted for.

\section{Discussion}

In Sec. 8 of this chapter, a simple conceptual approach to implicitly capture intrinsic ageing of the skin through variation of age-dependent constitutive parameters has been presented. This method was implemented and described in Sec. 9 where a subset of microstructural parameters associated with intrinsic ageing were identified from experimental characterisation data available in the literature. A microstructurally-motivated constitutive formulation featuring distinct free energy contributions from the ground substance, elastin and collagen phases was devised. The mechanical behaviour of the ground substance and elastin was modelled by an isotropic, coupled, and compressible neo-Hookean strain energy. For collagen, the primary load-bearing constituent of skin in tension, a wormlike eight-chain model 
motivated by the fibrous and intertwined nature of the collagen network was selected. This approach captures well the inherent nonlinearity and anisotropy of the skin under finite deformations.

A subset of the constitutive parameters whose age-dependent variation has the most physically-justified influence on the response was identified. The effects of ageing were successfully replicated through experimentally-informed alterations of these constitutive parameters. The reduction in skin stiffness at low stretches (i.e. when the equivalent Young's modulus is low) through degradation of the elastin network was well captured through modification of the elastic strain energy associated with elastin. Results show that degradation of the elastin mesh work and variations in anisotropy of the collagen network are plausible mechanisms to explain manifestations of ageing in terms of macroscopic tissue stiffening. Whereas alterations in elastin affect the low-strain region of the skin stress-strain curve, those related to collagen have an impact on its (large strain) linear region.

Despite the ability of the constitutive model to capture ageing effects, some limitations can be identified and form the basis for future improvements.

Only three sample points from experimental bulge test data were used, corresponding to ages 44,61 and 83 . This limited sampling space does not represent an adequate range of data with which to make a conclusive parameter fit. Therefore, for a more comprehensive ageing model to be developed, it would be necessary to include data from a statistically significant number of specimens and over a larger range of age groups. Moreover, skin specimens would need to be controlled for several factors so as to limit variability, or at least, to characterise it so it could be accounted for in the modelling. Of significant importance would be control over anatomical site. Not only should skin from the same site of the patients be tested, but also controlled for segregating the respective influence of intrinsic ageing and photoageing by considering photo-protected and photo-exposed zones.

The model does not explicitly account for the existence of crosslinks within the collagen network, and therefore, cannot provide a mechanistic description of their effect. Crosslinks prevent slippage between fibres and accordingly contributes toward the stiffness elicited by the collagen network under stretch [160]. With age, crosslinking has been observed to increase and this has often been considered as a possible reason for the increase in macroscopic stiffness [101]. It is worth pointing out that, in general, crosslinking of collagen might rather be a by-product of UV radiation exposure and/or glycation associated with diabetes [16] rather than the consequence of intrinsic ageing per se. Modifications of crosslinking properties are implicitly captured in the eight-chain model but modelling approaches explicitly accounting for crosslinks could also be considered. For example, for a more explicit description of fibre-to-fibre and matrix-to-fibre mechanical/physical interactions one could use dedicated tensor invariants $[161,162]$ that segregate deformation modes associated with such interactions, or one could use multiscale mechanistic micromechanical constitutive models that explicitly describe these interactions [163-166]. Micromorphic continuum models are also well suited to capture these effects $[167,168]$. Other types of microstructurally-motivated strain energy function for the individual chains of the unit cell could also be used $[169,170]$.

Because the dermal layer is the main mechanical contributor of the skin under states of tension it was reasonable to model the skin as single homogeneous layer. In order to build a more realistic model, valid for different load cases (e.g. surface shear), it would be sensible to incorporate individual skin layers with realistic structural geometries [20, 119] and associated constitutive properties.

Although the ageing skin undergoes significant alterations of its mechanical and biostructural properties, other body tissues, structures and organs undergo comparable ageing processes. As a result, they can alter the mechanical environment experienced by the skin. For example, thinning/atrophy of adipose and muscular tissues [101], or even bone resorption, are factors that can modify the complex mechanobiology and associated residual strains of the skin as an organ covering the entire body, and more particularly on the face. This is consistent with variations of Langer lines' tension and direction with age, reported in the literature [43]. These aspects were not directly accounted for in the present constitutive model and. Also, with age, the skin tends to lose its in-plane isotropy [102] because of the strong mechanical effects introduced by dermal collagen realignment arising in combination with collagen cross-linking and density alteration. These effects are also linked to the relaxation of tension in Langer lines with ageing.

At this stage, the model presented in Sec. 9 does not account for ageing-triggered enzymatic degradation of elastic fibres, abnormal collagen deposition and remodelling. This was addressed in Sec. 10 where, in the framework of opensystem thermodynamics and mixture theory, a biochemo-mechanical model of skin ageing was formulated based on a simplified mechanistic description of the biochemo-mechanics of both intrinsic and extrinsic ageing (Fig. 5) that was established in Sec. 4 of this chapter. The multiphysics constitutive model considered ROS, TGF- $\beta$, TIMP, MMP as 
biochemical species whose interplay modulated the homeostatic state of collagen and elastin phases. ROS were taken as the main triggers to initiate ageing of the skin. Density of ROS was the independent variable driving the ageing process. Of particular significance in this model, disruption of the homeostatic state between activation and inhibition of MMPs — through modulation of ROS and TIMP levels — was a key ingredient in describing the pathophysiology of intrinsic skin ageing and photoageing by controlling the degradation of collagen and elastin in the skin.

This prototype model should be viewed as a first attempt at partly deconstructing and simplifying the systemic characteristics of skin ageing. Here, we have laid down the foundation of a thermodynamically-admissible constitutive formulation embedded in the rigorous framework of continuum mechanics that capture ageing-induced evolution of fundamental biochemical species associated with ageing. The chemical interplay of these quantities (ROS, TGF- $\beta$, TIMP, MMP) modulates the evolution of microstructural characteristics of the skin, namely its collagen and elastin content. The model's ability to describe the evolution of collagen and elastin relative levels as a function of enzymatic factors and cytokines levels, themselves modulated by ROS levels, was demonstrated. It should be noted that, in its current formulation, the constitutive model does not encompass a two-way coupling between mechanics and biochemistry. This was an intentional feature, justified by the absence of suitable experimental data to calibrate such a model. The one-way coupling means that, although changes in collagen and elastin (relative) density as a by-product of ageing implicitly alter the composite properties of the skin, mechanics does not influence evolution of collagen and elastin levels or that of other biochemical constituents (ROS, TGF- $\beta$, TIMP, MMP). Moreover, a constrained mixture theory extension (Eq. (17)) was considered to effectively link evolution of collagen and elastin content to their respective contributions to the strain energy density defined in Eq. (7). Finally, a possible extension of the constitutive model to specifically include the effects of photoageing under the form of a weighted elastotic tissue component to the strain energy density (Eq. (18)).

Naturally, at this stage, much work remains to be done. Particular research efforts should be directed toward the design of physical characterisation experiments aiming to provide qualitative and quantitative evidence to support the formulation and validation of chemo-mechanobiological constitutive models of ageing. In Sec. 10 simple rate equations were chosen to describe the density evolution of all chemical species present in the mixture model. Equations of this type do not present any conceptual difficulty and more sophisticated analytical forms could have been used. However, the main challenge in developing, testing and validating biophysics-based model lies in the (limited) availability of experimental data, particularly biological fields (e.g. concentration of TIMPs as a function of age) to derive constitutive parameters.

The multiscale aspects of tissue structure - both in length and time scales than span several orders of magnitude - coupled to evolution of biochemical species present a significant technical challenge for modellers, particularly if considering computational coupling techniques [171].

In the light of this review of the main biomechanical and biostructural aspects of skin ageing, and despite a synthetic and simplified outlook, one is still left with a sense of overwhelming complexity. The number of processes and driving factors, their interplay, their multiphysics nature and the scales at which these occur make the understanding and description skin ageing a very challenging task, at both the experimental and modelling levels. Additionally, the effects of intrinsic ageing are generally compounded by the influence of extrinsic ageing (e.g. photoageing), which only serves to add complexity to the problem.

Here, in this chapter, we have outlined possible modelling approaches to capture the static or dynamic effects of ageing through the formulation of simple constitutive models of ageing. It is hoped that they will form the basis for the design of new physical characterisation experiments for skin ageing and more advanced multiphysics material models that will be calibrated with data produced in the context of such experiments. We strongly believe that only inter- and multidisciplinary approaches integrating experimental characterisation, imaging, clinical observation and modelling from the outset will have a real chance to deliver predictive tools to account for skin ageing in the design of treatment solutions and products. 


\section{Acknowledgements}

Part of this work was funded through the award of a Royal Society Newton Fund grant (2014-2016) between the Universities of Southampton and Cape Town. The authors would like to gratefully acknowledge this financial support as well as the logistic and infrastructure support provided by their respective institutions for research visits of Damien Pond, Andrew McBride and Georges Limbert. Georges Limbert would also like to thank Procter \& Gamble and L'Oréal for their financial support of his research over the last few years.

\section{References}

[1] T. Burns, S. Breathnach, N. Cox, and C. Griffiths. Rook's Textbook of Dermatology. Blackwell Science, Oxford, 7th edition edition, 2004. ISBN 0632064293.

[2] H. Shimizu. Shimizu's Textbook of Dermatology. Hokkaido University Press - Nakayama Shoten Publishers, 2007.

[3] D. J. Tobin. Biochemistry of human skin — our brain on the outside. Chemical Society Reviews, 35(1):52-67, 2006.

[4] H. Assaf, M. A. Adly, and M. R. Hussein. Aging and Intrinsic Aging: Pathogenesis and Manifestations, book section 13, pages 129-138. 2010.

[5] E. C. Naylor, R. E. B. Watson, and M. J. Sherratt. Molecular aspects of skin ageing. Maturitas, 69(3):249-256, 2011.

[6] B. A. Gilchrest and M. Yaar. Ageing and photoageing of the skin: observations at the cellular and molecular level. British Journal of Dermatology, 127 Suppl 41:25-30, 1992.

[7] D. Goukassian, F. Gad, M. Yaar, M. S. Eller, U. S. Nehal, and B. A. Gilchrest. Mechanisms and implications of the age-associated decrease in DNA repair capacity. FASEB J, 14(10):1325-34, 2000.

[8] G. J. Fisher, Z. Wang, S. C. Datta, J. Varani, S. Kang, and J. J. Voorhees. Pathophysiology of premature skin aging induced by ultraviolet light. New England Journal of Medicine, 337(20):1419-1429, 1997.

[9] M. Berneburg, H. Plettenberg, and J. Krutmann. Photoaging of human skin. Photodermatol Photoimmunol Photomed, 16(6):239-44, 2000.

[10] A. M. Kligman. Early destructive effects of sunlight on human skin. Journal of the American Medical Association, 210:2377-2380, 1969.

[11] G. J. Fisher, S. Kang, J. Varani, Z. Bata-Csorgo, Y. Wan, S. Datta, and J. J. Voorhees. Mechanisms of photoaging and chronological skin aging. Archives of Dermatology, 138(11):1462-70, 2002.

[12] B. L. Diffey. A quantitative estimate of melanoma mortality from ultraviolet a sunbed use in the uk. British Journal of Dermatology, 149(3):578-581, 2003.

[13] J. Sandby-Moller, T. Poulsen, and H. C. Wulf. Epidermal thickness at different body sites: relationship to age, gender, pigmentation, blood content, skin type and smoking habits. Acta Dermato Venereologica, 83(6):410-3, 2003.

[14] A. Vierkötter and J. Krutmann. Environmental influences on skin aging and ethnic-specific manifestations. Dermato-endocrinology, 4(3):227-231, 2012.

[15] A. V. Benedetto. The environment and skin aging. Clinical Dermatology, 16(1):129-139, 1998.

[16] M. J. Sherratt. Age-related tissue stiffening: Cause and effect. Advances in Wound Care, 2(1):11-17, 2013.

[17] F. H. Silver, G. Seehra, J. W. Freeman, and D. DeVore. Viscoelastic of young and old human dermis: a proposed moelcular mechanism for elastic energy storage in collagen and elastin. Journal of Applied Polymer Science, 86:1978-1985, 2002.

[18] M. F. Leyva-Mendivil, J. Lengiewicz, A. Page, N. W. Bressloff, and G. Limbert. Skin microstructure is a key contributor to its friction behaviour. Tribology Letters, 65(1):12, 2017.

[19] M. F. Leyva-Mendivil, J. Lengiewicz, A. Page, N. W. Bressloff, and G. Limbert. Implications of multi-asperity contact for shear stress distribution in the viable epidermis - an image-based finite element study. Biotribology, page in press, 2017. 
[20] M. F. Leyva-Mendivil, A. Page, N. W. Bressloff, and G Limbert. A mechanistic insight into the mechanical role of the stratum corneum during stretching and compression of the skin. Journal of the Mechanical Behavior of Biomedical Materials, 49:197-219, 2015.

[21] E. Hahnel, A. Lichterfeld, U. Blume-Peytavi, and J. Kottner. The epidemiology of skin conditions in the aged: A systematic review. Journal of Tissue Viability, 26(1):20-28, 2017.

[22] A. M. Kligman and C. Koblenzer. Demographics and psychological implications for the aging population. Dermatologic Clinics, 15(4):549-553, 1997.

[23] P. Morey. Skin tears: a literature review. Primary Intention, 15(3):122-129, 2007.

[24] 2010. URL http://www.statistics.gov.uk/cci/nugget.asp?id=949.

[25] www.ons.gov.uk/peoplepopulationandcommunity/populationandmigration/populationestimates/articles/ overviewoftheukpopulation/february2016, 2016.

[26] $2017 . \quad$ URL https://www.ons.gov.uk/peoplepopulationandcommunity/populationandmigration/ populationestimates/articles/overviewoftheukpopulation/july2017.

[27] United Nations. World population ageing. Department of Economic and Social Affairs Population Division, United Nations, New York, USA, ((ST/ESA/SER.A/390)):(ST/ESA/SER.A/390), 2015.

[28] E. Kelly, G. Stoye, and M. Vera-Hernandez. Public hospital spending in England: evidence from National Health Service administrative records. Technical report, Institute for Fiscal Studies, 2015.

[29] D. Pond, A. McBride, L. Davids, B. D. Reddy, and G. Limbert. Microstructurally-based constitutive modelling of the skin. linking intrinsic ageing to microstructural parameters. Journal of Theoretical Biology, 444:108-123, 2018.

[30] G. Limbert. State-of-the-art constitutive models of skin biomechanics, chapter 4, pages 95-131. Pan Stanford Publishing Pte. Ltd, Singapore, 2014.

[31] Y. Lanir. Skin Mechanics. McGraw-Hill, New York, 1987.

[32] L. S. Chan. Human skin basement membrane in health and autoimmune diseases. Frontiers in Bioscience, 2 (July 15):343-352, 1997.

[33] F. H. Silver, J. W. Freeman, and D. DeVore. Viscoelastic properties of human skin and processed dermis. Skin Research and Technology, 7(1):18-23, 2001.

[34] A. K. Langton, M. J. Sherratt, C. E. M. Griffiths, and R. E. B. Watson. Review article: A new wrinkle on old skin: the role of elastic fibres in skin ageing. International Journal of Cosmetic Science, 32(5):330-339, 2010.

[35] J. L. Lévêque, J. de Rigal, P. G. Agache, and C. Monneur. Influence of ageing on the in vivo extensibility of human skin at a low stress. Archives of Dermatological Research, 269(2):127-135, 1980.

[36] R. Reihsner, B. Balogh, and E. J. Menzel. Two-dimensional elastic properties of human skin in terms of an incremental model at the in vivo configuration. Medical Engineering and Physics, 17(4):304-313, 1995.

[37] H. Oxlund, J. Manschot, and A. Viidik. The role of elastin in the mechanical properties of skin. Journal of Biomechanics, 21(3):213-218, 1988.

[38] H. Oxlund and T. T. Andreassen. The roles of hyaluronic acid, collagen and elastin in the mechanical properties of connective tissues. Journal of Anatomy, 131(4):611-620, 1980.

[39] C. Pailler-Mattei, S. Bec, and H. Zahouani. In vivo measurements of the elastic mechanical properties of human skin by indentation tests. Medical Engineering \& Physics, 30(5):599-606, 2008.

[40] J. F. Ribeiro, E. H. M. dos Anjos, Maria Luiza S. Mello, and B. de Campos Vidal. Skin collagen fiber molecular order: a pattern of distributional fiber orientation as assessed by optical anisotropy and image analysis. PLOS ONE, 8(1):e54724, 2013.

[41] T. K. Tonge, L. S. Atlan, L. M. Voo, and T. D. Nguyen. Full-field bulge test for planar anisotropic tissues: Part I experimental methods applied to human skin tissue. Acta Biomaterialia, 9(4):5913-5925, 2013.

[42] J. Gosline, M. Lillie, E. Carrington, P. Guerette, C. Ortlepp, and K. Savage. Elastic proteins: biological roles and mechanical properties. Philosophical Transactions of the Royal Society of London. Series B: Biological Sciences, 357(1418):121-132, 2002.

[43] K. Langer. On the anatomy and physiology of the skin: II. Skin tension. British Journal of Plastic Surgery, 31 (2):93-106, 1978. 
[44] R. J. Lapeer, P. D. Gasson, and V. Karri. Simulating plastic surgery: From human skin tensile tests, through hyperelastic finite element models to real-time haptics. Progress in Biophysics and Molecular Biology, 103 (2-3):208-216, 2010.

[45] F. H. Silver, Y. P. Kato, M. Ohno, and A. J. Wasserman. Analysis of mammalian connective tissue: relationship between hierarchical structures and mechanical properties. Journal of long-term effects of medical implants, 2 (2-3):165-198, 1992.

[46] R. T. Tregear. The mechanical properties of skin. Journal of the Society of Cosmetic Chemists, 20:467-477, 1969.

[47] C. W. Oomens, D. H. van Campen, and H. J. Grootenboer. In vitro compression of a soft tissue layer on a rigid foundation. Journal of Biomechanics, 20(10):923-935, 1987.

[48] D. J. Tobin. Introduction to skin aging. Journal of Tissue Viability, 26(1):37-46, 2017.

[49] M. A. Farage, K. W. Miller, P. Elsner, and H. I. Maibach. Structural characteristics of the aging skin: A review. Cutaneous and Ocular Toxicology, 26(4):343-357, 2007.

[50] W. Montagna and K. Carlisle. Structural changes in ageing skin. Br J Dermatol, 122 Suppl 35:61-70, 1990.

[51] N. A. Fenske and C. W. Lober. Structural and functional changes of normal aging skin. Journal of the American Academy of Dermatology, 15(4(1)):571-585, 1986.

[52] N. Puizina-Ivić. Skin aging. Acta Dermatovenerologica Alpina Pannonica Adriatica, 17(2):47-54, 2008.

[53] A. Gragnani, S. MacCornick, V. Chominski, S.M. Ribeiro de Noronha, S. A. Alves Corra de Noronha, and L. Masako Ferreira. Review of major theories of skin aging. Advances in Aging Research, 3(4):265-284, 2014.

[54] J. Krutmann, A. Bouloc, G. Sore, B. A. Bernard, and T. Passeron. The skin aging exposome. Journal of Dermatological Science, 85(3):152-161, 2017.

[55] C. Escoffier, J. de Rigal, A. Rochefort, R. Vasselet, J. L. Lvłque, and P. G. Agache. Age-related mechanical properties of human skin: an in vivo study. The Journal of Investigative Dermatology, 93(3):353-357, 1989.

[56] M. Pawlaczyk, M. Lelonkiewicz, and M. Wieczorowski. Age-dependent biomechanical properties of the skin. Postepy Dermatologii i Alergologii, 30(5):302-306, 2013.

[57] S. Diridollou, V. Vabre, M. Berson, L. Vaillant, D. Black, J. M. Lagarde, J. M. Gregoire, Y. Gall, and F. Q. Patat. Skin ageing: changes of physical properties of human skin in vivo. International Journal of Cosmetic Science, 23(6):353-62, 2001.

[58] H. A. Oriba, D. A. Bucks, and H. I. Maibach. Percutaneous absorption of hydrocortisone and testosterone on the vulva and foreaarm: effect of the menopause and site. British Journal of Dermatology, 134:229-233, 1996.

[59] K. O. Duncan and D. J. Lefell. Preoperative assessment of eth elderly patient. Dermatologic Clinics, 15: 583-593, 1997.

[60] M. P. Brincat, S. Kabalan, J. W. Stud, C. F. Moniz, J. de Trafford, and J. Montgomery. A study of the decrease of skin collagen content, skin thickness, and bone mass in the postmeopausal women. Obstretic Gynecology, 70(6):840-845, 1987.

[61] B. A. Gilchrest. Age-associated changes in the skin. Journal of the American Geriatrics Society, 30(2):139-143, 1982.

[62] B.A. Gilchrest. Skin aging and photoaging: an overview. Journal of the American Academy of Dermatology, 21:610-613, 1989.

[63] F. Rippke, V. Schreiner, and H. Schwantitz. The acidic milieu of the horny layer: New findings on the physiology and pathophysiology of skin ph. American Journal of Clinical Dermatology, 3(4):261-272, 2002.

[64] J. M. Waller and H. I. Maibach. Age and skin structure and function, a quantitative approach (i): blood flow, ph, thickness, and ultrasound echogenicity. Skin Research and Technology, 11(4):221-35, 2005.

[65] H. Alexander and T. Cook. Variations with age in the mechanical properties of human skin in vivo. Journal of Tissue Viability, 16(3):6-11, 2006.

[66] F. Xu and T. Lu. Introduction to Skin Biothermomechanics and Thermal Pain. Springer, Heidelberg Dordrecht London New York, 2011.

[67] F. Henry, C. Piérard-Franchimont, G. Cauwenbergh, and G. E. Piérard. Age-related changes in facial skin contours and rheology. Journal of the American Geriatrics Society, 45(2):220-222, 1997.

[68] H. Tagami. Functional characteristics of the stratum corneum in photoaged skin in comparison with those found in intrinsic aging. Arch Dermatol Res, 300(1):S1-6, 2008. 
[69] F. H. Silver, L. M. Siperko, and G. P. Seehra. Mechanobiology of force transduction in dermal tissue. Skin Research and Technology, 9(1):3-23, 2003.

[70] R. McCallion and W. P. A. Li. Dry and photo-aged skin: manifestations and management. Journal of Clinical Pharmacology and Therapeutics, 18:15-32, 1993.

[71] M. Yaar, M. S. Eller, and B. A. Gilchrest. Fifty years of skin aging. Journal of Investigative Dermatology Symposium Proceedings, 7:51-58, 2002.

[72] D. Batisse, R. Bazin, T. Baldeweck, B. Querleux, and J. L. Lévêque. Influence of age on the wrinkling capacities of skin. Skin Research and Technology, 8(3):148-154, 2002.

[73] K. S. Wu, W. W. Van Osdol, and R. H. Dauskardt. Mechanical and microstructural properties of stratum corneum. Biological Biomimetic Materials-Properties to Function, 724:27-33, 2002.

[74] K. S. Wu, W. W. van Osdol, and R. H. Dauskardt. Mechanical properties of human stratum corneum: effects of temperature, hydration, and chemical treatment. Biomaterials, 27(5):785-95, 2006.

[75] L. H. Kligman. Photoaging. manifestations, prevention and treatment (review). Dermatologic Clinics, 4:517528, 1986.

[76] R. M. Lavker. Structural alterations in exposed and unexposed aged skin. Journal of Investigative Dermatology, 73:59-66, 1979.

[77] S. G. Lagarrigue, J. George, E. Questel, C. Lauze, N. Meyer, J. M. Lagarde, M. Simon, A. M. Schmitt, G. Serre, and C. Paul. In vivo quantification of epidermis pigmentation and dermis papilla density with reflectance confocal microscopy: variations with age and skin phototype. Experimental dermatology, 21(4):281-286, 2012.

[78] K. Sauermann, S. Clemann, S. Jaspers, T. Gambichler, P. Altmeyer, K. Hoffmann, and J. Ennen. Age related changes of human skin investigated with histometric measurements by confocal laser scanning microscopy in vivo. Skin Research and Technology, 8(1):52-56, 2002.

[79] B. Querleux, T. Baldeweck, S. Diridollou, J. de Rigal, E. Huguet, F. Leroy, and V. H. Barbosa. Skin from various ethnic origins and aging: an in vivo cross-sectional multimodality imaging study. Skin Research and Technology, 15(3):306-313, 2009.

[80] A. M. Kligman, P. Zheng, and R. M. Lavker. The anatomy and pathogenesis of wrinkles. British Journal of Dermatology, 113:37-42, 1985.

[81] R. Marks. Skin Disease in Old Sge. Martin Dunitz, London, second edition, 1999.

[82] G. L. Grove. Physiological changes in older skin. Clinical Geriatric Medicine, 5(1):115-125, 1989.

[83] J. H. Rabe, A. J. Mamelak, P. J. McElgunn, W. L. Morison, and D. N. Sauder. Photoaging: mechanisms and repair. J Am Acad Dermatol, 55(1):1-19, 2006.

[84] J. H. Chung, K. Yano, M. K. Lee, C. S. Youn, J. Y. Seo, K. H. Kim, K. H. Cho, H. C. Eun, and M. Detmar. Differential effects of photoaging vs intrinsic aging on the vascularization of human skin. Arch Dermatol, 138 (11):1437-42, 2002.

[85] P. Humbert, C. Viennet, K. Legagneux, F. Grandmottet, S. Robin, T. Oddos, and P. Muret. In the shadow of the wrinkle: theories. Journal of Cosmetic Dermatology, 11(1):72-78, 2012.

[86] C. R. Lovell, K. A. Smolenski, V. C. Duance, N. D. Light, S. Young, and M. Dyson. Type i and iii collagen content and fibre distribution in normal human skin during ageing. British Journal of Dermatology, 117(4): 419-428, 1987.

[87] M. Gniadecka, O. F. Nielsen, S. Wessel, M. Heidenheim, D. H. Christensen, and H. C. Wulf. Water and protein structure in photoaged and chronically aged skin. Journal of Investigative Dermatology, 111(6):1129-1132, 1998.

[88] J. Uitto. Connective tissue biochemistry of the aging dermis. age-associated alterations in collagen and elastin. Clinics in Geriatric Medicine, 5:127-147, 1989.

[89] G. J. Fisher, J. Varani, and J. J. Voorhees. Looking older: Fibroblast collapse and therapeutic implications. Archives of Dermatology, 144(5):666-672, 2008.

[90] J. Varani, M. K. Dame, L. Rittie, S. E. Fligiel, S. Kang, G. J. Fisher, and J. J. Voorhees. Decreased collagen production in chronologically aged skin: roles of age-dependent alteration in fibroblast function and defective mechanical stimulation. Am J Pathol, 168(6):1861-8, 2006.

[91] R. M. Lavker, P. Zheng, and G. Dong. Aged skin: a study by light, transmission electron, and scanning electron microscopy. Journal of Investigative Dermatology, 88(3):44-51, 1987. 
[92] R. M. Lavker, P. Zheng, and G. Dong. Morphology of aged skin. Journal of Geriatric Dermatology, 4(1): 53-67, 1989.

[93] J. Varani. Fibroblast aging: intrinsic and extrinsic factors. Drug Discovery Today: Therapeutic Strategies, 7(3): $65-70,2010$.

[94] W. S. Choi, A Mitsumoto, and I. E. Kochevar. Involvement of reactive oxygen species in tgf- $\beta 1$-induced tropoelastin expression by human dermal fibroblasts. Photochemistry and Photobiology, 85(6):1425-1433, 2009.

[95] J. L. Ashworth, G. Murphy, M. J. Rock, M. J. Sherratt, S. D. Shapiro, C. A. Shuttleworth, and C. M. Kielty. Fibrillin degradation by matrix metalloproteinases: implications for connective tissue remodelling. Biochemical Journal, 340(1):171-181, 1999.

[96] M. Yagi and Y. Yonei. Glycative stress and anti-aging: What is glycative stress? Glycative Stress Research, 3 (3):152-155, 2016.

[97] M. Yagi and Y. Yonei. Glycative stress and anti-aging: Glycative stress and skin aging. Glycative Stress Research, 5(1):50-54, 2018.

[98] G. J. Fisher, S. Datta, Z. Wang, X. Y. Li, T. Quan, J. H. Chung, S. Kang, and J. J. Voorhees. c-jun-dependent inhibition of cutaneous procollagen transcription following ultraviolet irradiation is reversed by all-trans retinoic acid. Journal of Clinical Investigation, 106(5):663-670, 2000.

[99] J. H. Chung, S. Kang, J. Varani, J. Lin, G. J. Fisher, and J. J. Voorhees. Decreased extracellular-signal-regulated kinase and increased stress-activated map kinase activities in aged human skin in vivo. Journal of Investigative Dermatology, 115(2):177-182, 2000.

[100] Colin H. Daly and George F. Odland. Age-related changes in the mechanical properties of human skin. Journal of Investigative Dermatology, 73(1):84-87, 1979.

[101] P. Quatresooz, L. Thirion, C. Piérard-Franchimont, and G. E. Piérard. The riddle of genuine skin microrelief and wrinkles. International Journal of Cosmetic Science, 28(6):389-395, 2006.

[102] E. C. Ruvolo Jr, G. N. Stamatas, and N. Kollias. Skin viscoelasticity displays site- and age-dependent angular anisotropy. Skin Pharmacology and Physiology, 20(6):313-321, 2007.

[103] A. Vexler, I. Polyansky, and R. Gorodetsky. Evaluation of skin viscoelasticity and anisotropy by measurement of speed of shear wave propagation with viscoelasticity skin analyzer. Journal of Investigative Dermatology, 113(5):732-739, 1999.

[104] I. E. Kochevar, C. R. Taylor, and J. Krutmann. Fundamentals of cutaneous photobiology and photoimmunology, chapter 88, pages 797-809. The McGraw-Hill Companies, New York, seventh edition, 2008.

[105] A. Anders, H.-J. Altheide, M. Knälmann, and H. Tronnier. Action spectrum for erythema in humans investigated with dye lasers. Photochemistry and Photobiology, 61(2):200-205, 1995.

[106] F. R. de Gruijl and J. C. van der Leun. Estimate of the wavelength dependency of ultraviolet carcinogenesis in humans and its relevance to the risk assessment of a stratospheric ozone depletion. Health Physics, 67(4): 319-325, 1994.

[107] B. Starcher and M. Conrad. A role for neutrophil elastase in the progression of solar elastosis. Connective Tissue Research, 31(2):133-140, 1995.

[108] E. F. Bernstein, Y. Q. Chen, K. Tamai, K. J. Shepley, K. S. Resnik, H. Zhang, R. Tuan, A. Mauviel, and J. Uitto. Enhanced elastin and fibrillin gene expression in chronically photodamaged skin. Journal of Investigative Dermatology, 103(2):182-186, 1994.

[109] M. Ramos-e Silva and S. C. da Silva Carneiro. Elderly skin and its rejuvenation: products and procedures for the aging skin. Journal of Cosmetic Dermatology, 6(1):40-50, 2007.

[110] R. E. Watson, C. E. Griffiths, N. M. Craven, C. A. Shuttleworth, and C. M. Kielty. Fibrillin-rich microfibrils are reduced in photoaged skin. distribution at the dermal-epidermal junction. J Invest Dermatol, 112(5):782-7, 1999.

[111] R. Fleischmajer, J. S. Perlish, and A. Gaisin. Comparative study of dermal glycosaminoglycans. Journal of Investigative Dermatology, 61(1):1-6, 1973.

[112] G. Jenkins. Molecular mechanisms of skin ageing. Mechanisms of Ageing and Development, 123(7):801-10, 2002. 
[113] J. C. Jeyapalan and J. M. Sedivy. Cellular senescence and organismal aging. Mechanisms of Ageing and Development, 129(7):467-474, 2008.

[114] C. López-Otín, M. A. Blasco, L. Partridge, M. Serrano, and G. Kroemer. The hallmarks of aging. Cell, 153(6): 1194-1217, 2013.

[115] S. Hekimi, J. Lapointe, and Y. Wen. Taking a good look at free radicals in the aging process. Trends in Cell Biology, 21(10):569-576, 2011.

[116] K. Merker, N. Sitte, and T. Grune. Hydrogen peroxide-mediated protein oxidation in young and old human mrc-5 fibroblasts. Archives of Biochemistry and Biophysics, 375(1):50-54, 2000.

[117] A. Goriely and M. Ben Amar. On the definition and modeling of incremental, cumulative, and continuous growth laws in morphoelasticity. Biomechanics and Modeling in Mechanobiology, 6(5):289-296, 2007.

[118] D. Ambrosi, G. A. Ateshian, E. M. Arruda, S. C. Cowin, J. Dumais, A. Goriely, G. A. Holzapfel, J. D. Humphrey, R. Kemkemer, E. Kuhl, J. E. Olberding, L. A. Taber, and K. Garikipati. Perspectives on biological growth and remodeling. Journal of the Mechanics and Physics of Solids, 59(4):863-883, 2011.

[119] G. Limbert. Mathematical and computational modelling of skin biophysics-a review. Proceedings of the Royal Society A-Mathematical Physical and Engineering Sciences, 473(2203):1-39, 2017.

[120] J. W. Y. Jor, M. D. Parker, A. J. Taberner, M. P. Nash, and P. M. F. Nielsen. Computational and experimental characterization of skin mechanics: identifying current challenges and future directions. Wiley Interdisciplinary Reviews: Systems Biology and Medicine, 5(5):539-556, 2013.

[121] Wenguang Li. Modelling methods for in vitro biomechanical properties of the skin: A review. Biomedical Engineering Letters, 5(4):241-250, 2015.

[122] C. Flynn. Fiber-matrix models of the dermis, book section 5, pages 133-159. Pan Stanford Publishing Pty Ltd, Singapore, 2014.

[123] E. Kuhl, K. Garikipati, E. Arruda, and K. Grosh. Remodeling of biological tissue: Mechanically induced reorientation of a transversely isotropic chain network. Journal of the Mechanics and Physics of Solids, 53: $1552-1573,2005$.

[124] E. Kuhl and G. A. Holzapfel. A continuum model for remodeling in living structures. Journal of Materials Science, 42(21):8811-8823, 2007.

[125] K. Garikipati, E. M. Arruda, K. Grosh, H. Narayanan, and S. Calve. A continuum treatment of growth in biological tissue: the coupling of mass transport and mechanics. Journal of the Mechanics and Physics of Solids, 52(7):1595-1625, 2004.

[126] E. Mazza, O. Papes, M. B. Rubin, S. R. Bodner, and N. S. Binur. Nonlinear elastic-viscoplastic constitutive equations for aging facial tissues. Biomechanics and Modeling in Mechanobiology, 4(2-3):178-189, 2005.

[127] E. Mazza, O. Papes, M. B. Rubin, S. R. Bodner, and N. S. Binur. Simulation of the aging face. Journal of Biomechanical Engineering-Transactions of the Asme, 129(4):619-623, 2007.

[128] M. B. Rubin and S. R. Bodner. A three-dimensional nonlinear model for dissipative response of soft tissue. International Journal of Solids and Structures, 39(19):5081-5099, 2002.

[129] F. Maceri, M. Marino, and G. Vairo. Age-dependent arterial mechanics via a multiscale elastic approach. International Journal for Computational Methods in Engineering Science and Mechanics, 14(2):141-151, 2013.

[130] J. E. Marsden and T. J. R. Hughes. Mathematical Foundations of Elasticity. Dover, New-York, 1994.

[131] E. M. Arruda and M. C. Boyce. A three-dimensional constitutive model for the large stretch behavior of rubber elastic-materials. Journal of the Mechanics and Physics of Solids, 41(2):389-412, 1993.

[132] P. J. Flory. Statistical mechanics of chain molecules. John Wiley \& Sons, Chichester-New York, 1969.

[133] J. E. Bischoff, E. A. Arruda, and K. Grosh. A microstructurally based orthotropic hyperelastic constitutive law. Journal of Applied Mechanics-Transactions of the ASME, 69(5):570-579, 2002.

[134] O. Kratky and G. Porod. Röntgenuntersuchungen gelöster fadenmoleküle. Recueil des Travaux Chimiques des Pays-Bas et de la Belgique, 68:1106-1122, 1949.

[135] A. Buganza Tepole, A. K. Gosain, and E. Kuhl. Stretching skin: The physiological limit and beyond. International Journal of Non-Linear Mechanics, 47(8):938-949, 2012.

[136] C. Flynn, A. J. Taberner, P. M. F. Nielsen, and S. Fels. Simulating the three-dimensional deformation of in vivo facial skin. Journal of the Mechanical Behavior of Biomedical Materials, 28(0):484-494, 2013. 
[137] C. Flynn and B. A. O. McCormack. A simplified model of scar contraction. Journal of Biomechanics, 41(7): $1582-1589,2008$.

[138] C. O. Flynn and B. A. O. McCormack. A three-layer model of skin and its application in simulating wrinkling. Computer Methods in Biomechanics and Biomedical Engineering, 12(2):125-134, 2009.

[139] J. E. Bischoff, E. M. Arruda, and K. Grosh. A rheological network model for the continuum anisotropic and viscoelastic behavior of soft tissue. Biomechanics and Modeling in Mechanobiology, 3(1):56-65, 2004.

[140] P. Sáez, E. Pea, M. A. Martnez, and E. Kuhl. Computational modeling of hypertensive growth in the human carotid artery. Computational Mechanics, 53(6):1183-1196, 2013.

[141] E. Kuhl, A. Menzel, and P. Steinmann. Computational modeling of growth - A critical review, a classification of concepts and two new consistent approaches. Computational Mechanics, 32(1-2):71-88, 2003.

[142] J. F. Marko and E. D. Siggia. Stretching dna. Macromolecules, 28(26):8759-8770, 1995.

[143] C. M. Kielty, M. J. Sherratt, and C. A. Shuttleworth. Elastic fibres. Journal of Cell Science, 115(14):2817-2828, 2002.

[144] T. K. Tonge, L. M. Voo, and T. D. Nguyen. Full-field bulge test for planar anisotropic tissues: Part II - a thin shell method for determining material parameters and comparison of two distributed fiber modeling approaches. Acta Biomaterialia, 9(4):5926-5942, 2013.

[145] J. W. Y. Jor, M. P. Nash, P. M. F. Nielsen, and P. J. Hunter. Estimating material parameters of a structurally based constitutive relation for skin mechanics. Biomechanics and Modeling in Mechanobiology, 10(5):767-778, 2011.

[146] Y. A. Kvistedal and P. M. F. Nielsen. Estimating material parameters of human skin in vivo. Biomechanics and Modeling in Mechanobiology, 8(1):1-8, 2009.

[147] G. Himpel, E. Kuhl, A. Menzel, and P. Steinmann. Computational modelling of isotropic multiplicative growth. Computer Modeling in Engineering and Sciences, 8(2):119-134, 2005.

[148] A. Menzel and E. Kuhl. Frontiers in growth and remodeling. Mechanics Research Communications, 42:1-14, 2012.

[149] C. R. Jacobs, M. E. Levenston, G. S. Beaupré, J. C. Simo, and D. R. Carter. Numerical instabilities in bone remodeling simulations: The advantages of a node-based finite element approach. Journal of Biomechanics, 28 (4), 1995.

[150] C. R. Jacobs, J. C. Simo, G. S. Beaupré, and D. R. Cartert. Adaptive bone remodeling incorporating simultaneous density and anisotropy considerations. Journal of Biomechanics, 30(96):603-613, 1997.

[151] V. Alastrué, J. F. Rodríguez, B. Calvo, and M. Doblaré. Structural damage models for fibrous biological soft tissues. International Journal of Solids and Structures, 44(18-19):5894-5911, 2007.

[152] E. Kuhl and P. Steinmann. Theory and numerics of geometrically non-linear open system mechanics. International Journal for Numerical Methods in Engineering, 58(11):1593-1615, 2003.

[153] T. Waffenschmidt, A. Menzel, and E. Kuhl. Anisotropic density growth of bone - A computational micro-sphere approach. International Journal of Solids and Structures, 49(14):1928-1946, 2012.

[154] Pierre Lafortune and R Aris. Computational model of collagen turnover in carotid arteries during hypertension. International Journal for Numerical Methods in Biomedical Engineering, 28(1):72-86, 2015.

[155] J. D. Humphrey and K. R. Rajagopal. A constrained mixture model for arterial adaptations to a sustained step change in blood flow. Biomechanics and Modeling in Mechanobiology, 2(2):109-126, 2003.

[156] A. Valentín and J. D. Humphrey. Evaluation of fundamental hypotheses underlying constrained mixture models of arterial growth and remodelling. Philosophical Transactions of the Royal Society A - Mathematical Physical and Engineering Sciences, 367(1902):3585-3606, 2009.

[157] A. Valentín, J. D. Humphrey, and G. A. Holzapfel. A multi-layered computational model of coupled elastin degradation, vasoactive dysfunction, and collagenous stiffening in aortic aging. Annals of Biomedical Engineering, 39(7):2027-2045, 2011.

[158] G Rouhi, M Epstein, L Sudak, and W Herzog. Modeling bone resorbtion using mixture theory with chemical reactions. Mechanics of Materials and Structures, 2(6):1141-1155, 2007.

[159] A. Valentín and G. A. Holzapfel. Constrained mixture models as tools for testing competing hypothesis in arterial biomechanics: Survey. Mechanics Research Communications, 29:126-133, 2012. 
[160] Y. Tang, R. Ballarini, M. J. Buehler, and S. J. Eppell. Deformation micromechanisms of collagen fibrils under uniaxial tension. Journal of the Royal Society Interface, 7(46):839-850, 2010.

[161] G. Limbert. A mesostructurally-based anisotropic continuum model for biological soft tissues-decoupled invariant formulation. Journal of the Mechanical Behavior of Biomedical Materials, 4(8):1637-1657, 2011.

[162] X. Q. Peng, Z. Y. Guo, and B. Moran. An anisotropic hyperelasticconstitutive model with fiber-matrix shear interaction for the human annulus fibrosus. Journal of Applied Mechanics, 73(5):815-824, 2005.

[163] F. Maceri, M. Marino, and G. Vairo. A unified multiscale mechanical model for soft collagenous tissues with regular fiber arrangement. Journal of biomechanics, 43(2):355-363, 2010.

[164] M. Marino and G. Vairo. Multiscale Elastic Models of Collagen Bio-structures: From Cross-Linked Molecules to Soft Tissues, pages 73-102. Springer Berlin Heidelberg, Berlin, Heidelberg, 2013.

[165] M. Marino, G. Vairo, and P. Wriggers. Multiscale hierarchical mechanics in soft tissues. Proceedings in Applied Mathematics and Mechanics, 15(1):35-38, 2015.

[166] K. Linka, V. N. Khiém, and M. Itskov. Multi-scale modeling of soft fibrous tissues based on proteoglycan mechanics. Journal of Biomechanics, 49(12):2349-2357, 2016.

[167] M. von Hoegen, S. Skatulla, and J. Schroder. A generalized micromorphic approach accounting for variation and dispersion of preferred material directions. Computers \& Structures, 2017.

[168] K. L. Sack, S. Skatulla, and C. C. Sansour. Biological tissue mechanics with fibres modelled as one-dimensional Cosserat continua. applications to cardiac tissue. International Journal of Solids and Structures, 81:84 - 94, 2016.

[169] G. A. Holzapfel, T. C. Gasser, and R. W. Ogden. A new constitutive framework for arterial wall mechanics and a comparative study of material models. Journal of Elasticity, 61:1-48, 2000.

[170] J. Schröder and P. Neff. Invariant formulation of hyperelastic transverse isotropy based on polyconvex free energy functions. International Journal of Solids and Structures, 40(2):401-445, 2003.

[171] M. G. D. Geers, V. G. Kouznetsova, and W. A. M. Brekelmans. Multi-scale computational homogenization: Trends and challenges. Journal of Computational and Applied Mathematics, 234(7):2175 - 2182, 2010. 